\title{
Generalized Robba rings
}

\author{
Gergely Zábrádi \\ with an appendix by Peter Schneider
}

9th April 2018

\begin{abstract}
We prove that any projective coadmissible module over the locally analytic distribution algebra of a compact $p$-adic Lie group is finitely generated. In particular, the category of coadmissible modules does not have enough projectives. In the Appendix a "generalized Robba ring" for uniform pro- $p$ groups is constructed which naturally contains the locally analytic distribution algebra as a subring. The construction uses the theory of generalized microlocalization of quasi-abelian normed algebras that is also developed there. We equip this generalized Robba ring with a self-dual locally convex topology extending the topology on the distribution algebra. This is used to show some results on coadmissible modules.
\end{abstract}

\section{Contents}

1 Introduction $\quad 2$

2 Preliminaries and basic notations 3

3 Projective coadmissible modules 4

3.1 Filtrations and the Grothendieck group . . . . . . . . . . . . . . 4

3.2 The Krull dimension and stable range . . . . . . . . . . . . . . . 7

4 Fréchet-Stein structure $\quad 10$

5 Topologies and self-duality $\quad 13$

5.1 The weak topology . . . . . . . . . . . . . . . . . . . . . . . . . . . . . . . . . . . .

5.2 The nice topology . . . . . . . . . . . . . . . . . . . . . . . 20

6 Further results on coadmissible modules $\quad 26$

A Appendix by Peter Schneider: Robba rings for compact $p$-adic Lie groups 32

A.1 Generalized microlocalization of quasi-abelian normed algebras . . . . . . . . . 32

A.2 Noncommutative annuli for uniform pro- $p$-groups . . . . . . . . . . . . . . . . 46

A.3 Bounded rings . . . . . . . . . . . . . . . . . . . . . . 51 


\section{Introduction}

The theory of locally analytic representations of $p$-adic analytic groups has recently been developed by Schneider and Teitelbaum [ST1, ST2, ST3, ST4, ST5, ST7]. The category $\mathcal{C}_{D(G, K)}$ of coadmissible modules over the $K$-valued locally analytic distribution algebra $D(G, K)$ plays a crucial role in the theory. Here $\mathbb{Q}_{p} \subseteq K \subseteq \mathbb{C}_{p}$ is a complete and discretely valued field and $G$ a compact $p$-adic Lie group. A locally analytic representation of $G$ over the field $K$ is called admissible if its strong dual is a coadmissible module over $D(G, K)$. (Locally analytic representations of non-compact $p$-adic Lie groups are called admissible if their restriction to a - or equvialently, to any - compact open subgroup is admissible.) The distribution algebra $D(G, K)$ is a Fréchet-Stein algebra as it is the projective limit of the noetherian Banach algebras $D_{r_{l}}(G, K)(l \geq 1)$ with flat connecting maps. Here the algebras $D_{r_{l}}(G, K)$ are certain completions of $D(G, K)$ with respect to norms $r_{l}$ (for details see section 2).

The finitely presented modules are always coadmissible, but the coadmissible modules over $D(G, K)$ need not even be finitely generated, and there exist finitely generated $D(G, K)$ modules that are not coadmissible. However, in this paper we show that all the projective objects in $\mathcal{C}_{D(G, K)}$ are finitely generated (and then, of course, also finitely presented) generalizing earlier results in the commutative case by Gruson [G]. In particular, the category $\mathcal{C}_{D(G, K)}$ does not have enough projective objects. As a by-product we also compute the Grothendieck group $K_{0}$ of the finitely generated projective $D_{r}(G, K)$-modules whenever $G$ is uniform.

However, the main aim of this paper is to investigate the properties of the so-called "generalized Robba ring" that is constructed in the Appendix of this paper. The $\operatorname{ring} \mathcal{R}(G, K)$ (the generalized Robba ring) is defined as follows. Using the theory of generalized microlocalization (see the Appendix) one obtains "closed noncommutative annuli" for uniform pro- $p$ groups, ie. rings $D_{\left[\rho, \rho^{\prime}\right]}(G, K)$ (with $p^{-1}<\rho<\rho^{\prime}<1$ ) whose elements are interpreted as noncommutative Laurent series converging on a closed $p$-adic polyannulus $\rho \leq\left|T_{1}\right|=\cdots=\left|T_{d}\right| \leq \rho^{\prime}$ (with $d$ being the dimension of the uniform pro- $p$ group $G$ ). As it is shown in section 4 , these are noetherian Banach algebras (whenever $\rho$ and $\rho^{\prime}$ are rational powers of $p$ ). One then takes the projective limit with respect to the natural flat (cf. Thm. 4.3) connecting maps $D_{\left[\rho, \rho_{2}\right]}(G, K) \hookrightarrow D_{\left[\rho, \rho_{1}\right]}(G, K)$ (for $\left.p^{-1}<\rho<\rho_{1}<\rho_{2}<1\right)$ to obtain the Fréchet-Stein algebras $D_{[\rho, 1)}(G, K)$. The generalized Robba ring is then the inductive limit of these Fréchet-Stein algebras when $\rho$ tends to 1 . The $\operatorname{ring} \mathcal{R}(G, K)$ admits a self-dual ring topology such that $\operatorname{Hom}_{K}^{c t}(\mathcal{R}(G, K), K) \cong \mathcal{R}(G, K)$ as topological $\mathcal{R}(G, K)$-modules. The self-dual topology on $\mathcal{R}(G, K)$ is unfortunately not equal to the inductive limit topology of the Fréchet topologies on $D_{[\rho, 1)}(G, K)$ as the latter is not self-dual (see Corollary 5.13) with respect to the pairing constructed in section 5. However, the topology we construct (the "nice" topology) has the following properties:

(i) The ring $\mathcal{R}(G, K)$ is self-dual in the nice topology with respect to the pairing

$$
\begin{aligned}
\mathcal{R}(G, K) \times \mathcal{R}(G, K) & \rightarrow K \\
(x, y) & \mapsto \text { "the constant term of } x y . "
\end{aligned}
$$

(ii) On the classical Robba $\operatorname{ring} \mathcal{R}\left(\mathbb{Z}_{p}, K\right)$ the nice topology coincides with the inductive limit topology. 
(iii) The subspace topology on the distribution algebra $D(G, K)$ equals the usual Fréchet topology.

In the last section of this paper we use the Fréchet-Stein structure of the algebras $D_{[\rho, 1)}(G, K)$ to construct an exact functor $R$ from the category of coadmissible modules over $D(G, K)$ to the category of modules over the generalized Robba ring $\mathcal{R}(G, K)$. We call the image of this functor the category of coadmissible modules over $\mathcal{R}(G, K)$. Our main result (Theorem 6.6) in this section states that whenever $M$ is a finitely generated coadmissible module over $D(G, K)$ then $\operatorname{Hom}_{K}^{c t}(R(M), K)$ is also coadmissible where the topology on $R(M)$ is a canonical topology coming from the nice topology of $\mathcal{R}(G, K)$. By Proposition 3.10 this includes all the projective objects in the category.

\section{Acknowledgements}

I gratefully acknowledge the hospitality of the Westfälische Wilhelmsuniversität Münster where the most of this paper was written. During this research I was supported by the German Israeli Foundation for Research and Development and by the Deutsche Forschungsgemeinschaft (DFG). I am mostly indebted to Peter Schneider for introducing me to the representation theory of $p$-adic groups, for drawing my attention to Robba rings, for his valuable comments, and for reading earlier versions of the manuscript so carefully. I would also like to thank Tobias Schmidt for reading parts of the manuscript and for various discussions about the topic. I am grateful to the referee for his careful reading of the manuscript, for his remarks on the presentation of results, and for simplifying the proof of Thm. 5.5.

\section{Preliminaries and basic notations}

Let $p$ be a prime number and let $\kappa$ be 1 if $p$ is odd and 2 if $p=2$. Let $\mathbb{Q}_{p} \subseteq K$ be a complete and discretely valued field, and $k$ denotes the residue field of $K$. Further, let $G$ be a compact locally $\mathbb{Q}_{p}$-analytic group which we will assume for the most of the paper to be uniform.

For any compact locally $\mathbb{Q}_{p}$-analytic group $G$ we denote by $D(G, K)$ the algebra of $K$ valued locally analytic distributions on $G$. Recall that $D(G, K)$ is equal to the strong dual of the locally convex vector space $C^{a n}(G, K)$ of $K$-valued locally $\mathbb{Q}_{p}$-analytic functions on $G$ with the convolution product.

Now if $G$ is uniform then it has a bijective global chart

$$
\begin{aligned}
\mathbb{Z}_{p}^{d} & \rightarrow G \\
\left(x_{1}, \ldots, x_{d}\right) & \mapsto h_{1}^{x_{1}} \cdots h_{d}^{x_{d}}
\end{aligned}
$$

where $h_{1}, \ldots, h_{d}$ is a fixed (ordered) minimal set of topological generators of $G$. Putting $b_{i}:=h_{i}-1 \in \mathbb{Z}[G], \mathbf{b}^{\alpha}:=b_{1}^{\alpha_{1}} \cdots b_{d}^{\alpha_{d}}$ for $\alpha \in \mathbb{N}_{0}^{d}$ we can identify $D(G, K)$ with the ring of all formal series

$$
\lambda=\sum_{\alpha \in \mathbb{N}_{0}^{d}} d_{\alpha} \mathbf{b}^{\alpha}
$$

with $d_{\alpha}$ in $K$ such that the set $\left\{\left|d_{\alpha}\right| \rho^{\kappa|\alpha|}\right\}_{\alpha}$ is bounded for all $0<\rho<1$. Here the first $|\cdot|$ is the normalized absolute value on $K$ and the second one denotes the degree of $\alpha$, that is 
$\sum_{i} \alpha_{i}$. Note that this is different from the convention in the Appendix, where $|\alpha|$ is defined as $\sum_{i}\left|\alpha_{i}\right|$. For any $\rho$ in $p^{\mathbb{Q}}$ with $p^{-1}<\rho<1$, we have a multiplicative norm $\|\cdot\|_{\rho}$ on $D(G, K)$ [ST5] given by

$$
\|\lambda\|_{\rho}:=\sup _{\alpha}\left|d_{\alpha}\right| \rho^{\kappa|\alpha|} .
$$

The family of norms $\|\cdot\|_{\rho}$ defines the Fréchet topology on $D(G, K)$. The completion with respect to the norm $\|\cdot\|_{\rho}$ is denoted by $D_{\rho}(G, K)$. (Whenever $G$ is not uniform then we choose an open normal uniform subgroup $H$ and representatives $g_{1}, \ldots, g_{G: H}$ for the cosets in $G / H$ we can define the norms $\|\cdot\|_{\rho}$ on $D(G, K)=\bigoplus_{i} D(H, K) g_{i}$ by $\left\|\sum_{i} \lambda_{i} g_{i}\right\|_{\rho}:=\max _{i}\left\|\lambda_{i}\right\|_{\rho}$ and denote by $D_{\rho}(G, K)$ the corresponding completions.)

Now if we fix real numbers $p^{-1}<\rho_{1} \leq \cdots \leq \rho_{l} \leq \cdots<1$ in $p^{\mathbb{Q}}$ such that $\rho_{l} \rightarrow 1$ then the distribution algebra $D(G, K)$ is the projective limit of the noetherian Banach-algebras $D_{\rho_{l}}(G, K)$. This gives the Fréchet-Stein algebra structure on $D(G, K)$. A coherent sheaf for the projective system $\left(D_{\rho_{l}}(G, K), l\right)$ of algebras is a projective system of finitely generated modules $M_{l}$ over $D_{\rho_{l}}(G, K)$ together with isomorphisms

$$
D_{\rho_{l}}(G, K) \otimes_{D_{\rho_{l+1}}(G, K)} M_{l+1} \stackrel{\cong}{\rightrightarrows} M_{l}
$$

for any positive integer $l$. Following [ST5] we call a $D(G, K)$-module $M$ coadmissible if it is isomorphic to the $D(G, K)$-module of "global sections"

$$
\Gamma\left(M_{l}\right):=\lim _{l} M_{l}
$$

of a coherent sheaf $\left(M_{l}\right)_{l}$ for $\left(D(G, K), \rho_{l}\right)$. A coadmissible $D(G, K)$-module need not be finitely generated, neither is a finitely generated module always coadmissible. For example if one takes an ideal $I$ in $D\left(\mathbb{Z}_{p}, K\right)$ that is not closed (or equivalently, not finitely generated) then the quotient $D\left(\mathbb{Z}_{p}, K\right) / I$ is finitely generated (even cyclic), but not coadmissible. On the other hand put $M:=\prod_{i=1}^{\infty} D\left(\mathbb{Z}_{p}, K\right) /\left(\frac{\log (1+x)}{(x+1)^{p^{i}}-1}\right)$ as a module over $D\left(\mathbb{Z}_{p}, K\right)$. Since the zeroes of $\log (1+x)$ are exactly of the form $\zeta-1$ with $\zeta \in \mu_{p \infty}$, the restriction of $M$ to any closed disc of radius $\rho<1$ is finitely generated. Hence $M$ is coadmissible as it is clearly the projective limit of its restrictions to closed discs of radius $\rho<1$. Although, $M$ is not finitely generated, since its localisation $M_{\zeta_{p} m-1}$ at the point corresponding to the Galois orbit of $\zeta_{p^{m}}-1$ has rank $m-1$ for any $m \geq 1$ with $\zeta_{p^{m}}$ being a primitive $p^{m}$ th root of unity. However, any finitely presented module is coadmissible. For further details see [ST5].

\section{Projective coadmissible modules}

\subsection{Filtrations and the Grothendieck group}

The ring $D_{\rho}(G, K)$ is a filtered left and right noetherian $K$-Banach algebra realizing the Fréchet-Stein structure on $D(G, K)$. The filtration is given by the additive subgroups

$$
\begin{aligned}
F_{\rho}^{s} D_{\rho}(G, K) & :=\left\{\lambda \in D_{\rho}(G, K):\|\lambda\|_{\rho} \leq p^{-s}\right\}, \\
F_{\rho}^{s+} D_{\rho}(G, K) & :=\left\{\lambda \in D_{\rho}(G, K):\|\lambda\|_{\rho}<p^{-s}\right\}
\end{aligned}
$$


for $s$ in $\mathbb{R}$ with associated graded ring

$$
\operatorname{gr}^{\cdot} D_{\rho}(G, K):=\bigoplus_{s \in \mathbb{R}} F_{\rho}^{s}\left(D_{\rho}(G, K)\right) / F_{\rho}^{s+} D_{\rho}(G, K) .
$$

It is proven in [ST5] that gr $D_{\rho}(G, K)$ is isomorphic to the polynomial $\operatorname{ring}(\operatorname{gr} K)\left[X_{1}, \ldots, X_{d}\right]$ and the principal symbols $\sigma\left(b_{i}\right)$ of $b_{i}$ are the variables $X_{i}$ provided that $\rho$ lies in $p^{\mathbb{Q}}$. The graded ring of the field $K$ is naturally isomorphic to the Laurent polynomial ring $k\left[X_{0}, X_{0}^{-1}\right]$ over the residue field $k$ of $K$. The uniformizer maps to the variable $X_{0}$ under gr:

Lemma 3.1. Any graded projective module over the ring gr $D_{\rho}(G, K)$ is stably graded free.

Proof. This is a classical statement proven in [G] (§1, Theorem 1).

Now we claim that $D_{\rho}(G, K)$ satisfies the invariant basis property. Indeed, $D_{\rho}(G, K)$ is left and right Noetherian ring without zero divisors so it has a full skew field of fractions (by Goldie's Theorem). Hence the rank of a finitely generated free module is well-defined.

For any real number $s$ such that $F_{\rho}^{s} D_{\rho}(G, K)$ is not equal to $F_{\rho}^{s+} D_{\rho}(G, K)$ we denote by $D_{\rho}(G, K)(s)$ the module $D_{\rho}(G, K)$ over itself together with the shifted filtration

$$
\begin{aligned}
F_{\rho}^{t} D_{\rho}(G, K)(s) & :=F_{\rho}^{t+s} D_{\rho}(G, K) \\
F_{\rho}^{t+} D_{\rho}(G, K)(s) & :=F_{\rho}^{(t+s)+} D_{\rho}(G, K) .
\end{aligned}
$$

We call a finitely generated module filtered free if it is the (filtered) direct sum of modules of the form $D_{\rho}(G, K)(s)$. Let $\mathcal{C}_{0}$ be the category of those finitely generated filtered projective $D_{\rho}(G, K)$-modules $P$ that are direct summands of some filtered free modules of finite rank as filtered modules. Further we denote by $\mathcal{C}$ the category of admissibly filtered finitely generated $D_{\rho}(G, K)$-modules. We call a filtration on a module $M$ admissible if it is the image of the filtration of a filtered free module under a surjective homomorphism onto $M$. Note that any finitely generated $D_{\rho}(G, K)$-module admits an admissible filtration. Moreover, the category $\mathcal{C}$ is abelian by the following lemma.

Lemma 3.2. The induced filtration on any submodule of a finitely generated filtered free module is admissible.

Proof. Let $M$ be a submodule of the filtered free module $F$. Then $\operatorname{gr}^{\circ} M$ is a submodule of the finitely generated graded free module gr $F$. So gr $M$ is generated by a finite set of homogeneous elements in gr $F$. We can lift up these homogeneous elements to $M$ and denote the lifts by $m_{1}, \ldots, m_{l}$. We claim first that the elements $m_{i}$ generate the module $M$. Indeed, $D_{\rho}(G, K)$ is complete with respect to the filtration and for any element $m$ in $M$ we can choose a sum $\sum_{i} a_{i} m_{i}$ such that the projections to the graded module $\sigma(m)$ and $\sigma\left(\sum_{i} a_{i} m_{i}\right)$ are equal. On the other hand since $D_{\rho}(G, K)$ is a noetherian Banach algebra, any submodule of a finitely generated module is closed and the claim follows. Now it suffices to prove that the filtration on $M$ is the induced by the surjection from $F_{1}:=\bigoplus_{i} D_{r}(G, K)\left(s_{i}\right)$ sending the generator of $D_{\rho}(G, K)\left(s_{i}\right)$ to $m_{i}$. Here $s_{i}$ is the degree of $m_{i}$. By construction we have for any real number $s$ that the image of $F_{\rho}^{s} F_{1}$ is contained in $F_{r}^{s} M$. On the other hand if $m$ is in $F_{r}^{s} M \backslash F_{r}^{s+} M$ then $\sigma(m)$ is a homogeneous element of gr $M$ of degree $s$. So it can be written in the form

$$
\sigma(m)=\sum_{i} b_{i} \sigma\left(m_{i}\right)
$$


such that $b_{i} \sigma\left(m_{i}\right)$ is also homogeneous of degree $s$. By a similar argument as above using the completeness of $M$ with respect to the filtration the result follows.

Lemma 3.3. Let $F$ be a finitely generated filtered free $D_{\rho}(G, K)$-module and $\pi$ be a homogeneous projection of gr $F$ of degree 0 . Then $\pi$ can be lifted to a projection of $F$.

Proof. It is easy to see that one can lift $\pi$ up to an endomorphism $g$ of the module $F$ as it suffices to give $g$ on the free generators $e_{i}(i=1, \ldots, \operatorname{rk}(F))$ such that $\sigma\left(g\left(e_{i}\right)\right)=\pi\left(\sigma\left(e_{i}\right)\right)$. Now let $B$ be the closed subring of $\operatorname{End}_{D_{\rho}(G, K)}(F)$ generated by $g$. Then $B$ is a commutative positively filtered ring that is complete with respect to the filtration. Moreover, the element $g-g^{2}$ has positive degree. Hence taking the ideal $I$ of elements of positive degree and applying Chapter III, §4, Lemma 2 in [B-AC] one obtains an element $g_{1}$ in $B$ with $g_{1}=g_{1}^{2}$ and $g-g_{1}$ is in $I$.

Lemma 3.4. The natural inclusion from $\mathcal{C}_{0}$ to $\mathcal{C}$ induces an isomorphism $K_{0}\left(\mathcal{C}_{0}\right) \rightarrow K_{0}(\mathcal{C})$ on the Grothendieck groups.

Proof. It is easy to see (theorem 4 and 5 in $[\mathrm{BHS}]$ ) that it suffices to show that each module in $\mathcal{C}$ has a finite resolution by elements in $\mathcal{C}_{0}$. Now using Lemma 3.2 any finitely generated admissibly filtered $D_{\rho}(G, K)$-module $M$ has a filtered-free resolution

$$
\cdots \rightarrow F_{j} \stackrel{d_{j}}{\rightarrow} \cdots \rightarrow F_{0} \rightarrow M \rightarrow 0
$$

by finitely generated modules as $D_{\rho}(G, K)$ is noetherian. Moreover, the functor gr is exact and gives a free resolution

$$
\cdots \rightarrow \operatorname{gr} F_{j} \stackrel{\operatorname{gr}^{\cdot} d_{j}}{\rightarrow} \cdots \rightarrow \operatorname{gr} \cdot F_{0} \rightarrow \operatorname{gr} M \rightarrow 0 .
$$

The global dimension of gr $D_{\rho}(G, K)$ is finite and bounded by its Krull dimension. This means that there is a positive integer $j$ such that $\operatorname{Ker}\left(\operatorname{gr} d_{j}\right)=\operatorname{gr} \operatorname{Ker}\left(d_{j}\right)$ is projective. Hence we have a section $\beta$ from $\operatorname{Ker}\left(\operatorname{gr}^{\cdot} d_{j}\right)$ to gr $F_{j+1}$. By Lemma 3.3 the projection $\beta \circ$ gr $d_{j+1}$ of gr $F_{j+1}$ can be lifted to a projection $g$ of $F_{j+1}$. Therefore $g\left(F_{j+1}\right)$ is in the category $\mathcal{C}_{0}$ and it maps bijectively onto $\operatorname{Ker}\left(d_{j}\right)$ under the map $d_{j+1}$ and we are done.

Proposition 3.5. Let $1 / p<\rho<1$ be in $p^{\mathbb{Q}}$. Then $K_{0}\left(D_{\rho}(G, K)\right) \cong \mathbb{Z}$, in other words all the projective $D_{\rho}(G, K)$-modules are stably free.

Proof. By Lemma 3.4 it is enough to prove that any object in $\mathcal{C}_{0}$ is stably filtered free as the map

$$
K_{0}(\mathcal{C}) \rightarrow K_{0}\left(D_{\rho}(G, K)\right)
$$

induced by the forgetful functor is clearly surjective and $D_{\rho}(G, K)$ has the invariant basis property. Now if $P$ is in $\mathcal{C}_{0}$ then the associated graded module gr $P$ is also projective, so by Lemma 3.1 it is stably graded free. Since gr is exact, $P$ is also stably filtered free and we are done. 


\subsection{The Krull dimension and stable range}

Whenever $R$ is a (not necessarily commutative) ring then following [GaRe] its right Krull dimension $\operatorname{Kdim} R$ is defined by the deviation of the poset of right ideals in $R$. For the deviation of a poset see Chapter 6 in [MR. By Corollary 4.8 in [MR this definition of Krull dimension coincides with the usual one whenever $R$ is commutative. Since distribution algebras of groups are isomorphic to their opposite ring via the map induced by the anti-involution on the group sending the elements to their inverse, the right and left Krull dimensions of $D_{\rho}(G, K)$ are the same and we just call this the Krull dimension $\operatorname{Kdim} D_{\rho}(G, K)$ of $D_{\rho}(G, K)$. A priori this is just an ordinal, but we shall show that $\operatorname{Kdim} D_{\rho}(G, K)$ is finite.

Lemma 3.6. The Krull dimension of the ring $D_{\rho}(G, K)$ is less than or equal to $d+1=$ $\operatorname{Kdim}\left(\operatorname{gr} D_{\rho}(G, K)\right)$.

Proof. This follows from Proposition 7.1.2 of Chapter I of [LvO] and Corollary 6.4.8 of [MR] as $D_{\rho}(G, K)$ is complete with respect to the filtration.

Now we aim at studying the projective objects in the category of coadmissible $D(G, K)$ modules. For this we introduce the notion of stable range following [Ba]. Let $R$ be a ring and we denote by $\mathrm{GL}(R)$ the infinite dimensional general linear group $\lim _{n} \mathrm{GL}_{n}(R)$ and by $\mathrm{E}(R)$ its subgroup generated by elementary matrices. Then we have

$$
\begin{aligned}
\mathrm{E}(R) & \cong[\mathrm{GL}(R), \mathrm{GL}(R)] \text { and } \\
K_{1}(R) & \cong \mathrm{GL}(R) / \mathrm{E}(R) .
\end{aligned}
$$

Let $a=\left(a_{1}, \ldots, a_{l}\right)$ be an element in the right module $R^{l}$. We call $a$ unimodular if there is a homomorphism $f: R \rightarrow R$ such that $f(a)=1$ (this is equivalent in this special case to that the left ideal $R a_{1}+\cdots+R a_{l}$ equals $R$ ). We say that the positive integer $n$ defines a stable range for $\mathrm{GL}(R)$ if, for all $l>n$, given $\left(a_{1}, \ldots, a_{l}\right)$ unimodular in $R^{l}$, there exist $b_{1}, \ldots, b_{l-1}$ in $R$ such that $\left(a_{1}+b_{1} a_{l}, \ldots, a_{l-1}+b_{l-1} a_{l}\right)$ is unimodular in $R^{l-1}$.

Corollary 3.7. The number $d+2$ defines a stable range for $\operatorname{GL}\left(D_{\rho}(G, K)\right)$. Any projective $D_{\rho}(G, K)$-module of rank at least $d+2$ is free. In particular if $P$ is a finitely generated projective module then it can be generated by $\max (\operatorname{rk}(P), d+2)$ elements.

Proof. The first statement follows from Lemma 3.6 and the Theorem in St] stating that if the Krull dimension of a right noetherian ring $R$ is at most $n-1$ then $n$ defines a stable range for $\operatorname{GL}(R)$. Now by Theorem 4.2 in $\left[\mathrm{Ba}\right.$ ] the group $\mathrm{E}_{d+3}\left(D_{\rho}(G, K)\right)$ generated by elementary $(d+3) \times(d+3)$ matrices is surjective on the unimodular rows in $D_{\rho}(G, K)^{d+3}$. So if $P$ is a projective module such that

$$
P \oplus F \cong D_{\rho}(G, K)^{t}
$$

for some integer $t \leq d+3$ and $F \cong D_{\rho}(G, K)$ then there is an automorphism $g$ of $D_{\rho}(G, K)^{t}$ sending the generator of $F$ to the first basis vector in $D_{\rho}(G, K)^{t}$ as the generator of $F$ is unimodular. So we obtain

$$
P \cong(P \oplus F) / F \stackrel{g}{\cong} D_{\rho}(G, K)^{t} / D_{\rho}(G, K) \cong D_{\rho}(G, K)^{t-1} .
$$

The result follows by Proposition 3.5 . 
Lemma 3.8. Let $G$ be a uniform pro-p group of dimension $d$. Then for any positive integer $m$ the subgroup $\mathrm{E}_{m+d+2}\left(D_{\rho}(G, K)\right)$ of $\mathrm{GL}_{m+d+2}\left(D_{\rho}(G, K)\right)$ generated by elementary matrices acts transitively on the surjections from $D_{\rho}(G, K)^{m+d+2}$ to $D_{\rho}(G, K)^{m}$.

Proof. Fix a basis of both $D_{\rho}(G, K)^{m+d+2}$ and $D_{\rho}(G, K)^{m}$. Let $A=\left(a_{i j}\right)_{1 \leq i \leq m, 1 \leq j \leq m+d+2}$ be the matrix of a surjection. We need to show that there is a matrix $B$ in $\mathrm{E}_{m+d+2}\left(D_{\rho}(G, K)\right)$ such that we have

$$
A B=\left(\begin{array}{cccccccc}
1 & 0 & 0 & \cdots & 0 & 0 & \cdots & 0 \\
0 & 1 & 0 & \cdots & 0 & 0 & \cdots & 0 \\
\vdots & \ddots & \ddots & \ddots & \vdots & \vdots & & \vdots \\
0 & \cdots & 0 & 1 & 0 & 0 & \cdots & 0 \\
0 & \cdots & \cdots & 0 & 1 & 0 & \cdots & 0
\end{array}\right)
$$

is the standard surjection. By Theorem 4.2a) in $[\mathrm{Ba}] \mathrm{E}_{m+d+2}\left(D_{\rho}(G, K)\right)$ acts transitively on unimodular rows since $d+2$ defines a stable range by Corollary 3.7. On the other hand the first (or in fact any) row of $A$ is unimodular as $A$ is surjective. This means that we may assume without loss of generality that the first row of $A$ is

$$
\left(\begin{array}{llll}
1 & 0 & \cdots & 0
\end{array}\right) \text {. }
$$

Using this above remark we prove the statement by induction on $m$. If $m=1$ then we are done by the above argument. Now let $m>1$ and suppose the statement is true for $m-1$. Since the first row of $A$ is as in (44) we have that the matrix $A^{\prime}=\left(a_{i j}\right)_{2 \leq i \leq m, 2 \leq j \leq m+d+2}$ is also a surjection. By the inductional hypothesis there is a matrix $B$ in $\mathrm{E}_{m+d+1}\left(D_{\rho}(G, K)\right)$ such that

$$
A\left(\begin{array}{cc}
1 & 0 \\
0 & B
\end{array}\right)=\left(\begin{array}{cccccccc}
1 & 0 & 0 & \cdots & 0 & 0 & \cdots & 0 \\
* & 1 & 0 & \cdots & 0 & 0 & \cdots & 0 \\
\vdots & 0 & \ddots & \ddots & \vdots & \vdots & & \vdots \\
* & \vdots & \ddots & 1 & 0 & 0 & \cdots & 0 \\
* & 0 & \cdots & 0 & 1 & 0 & \cdots & 0
\end{array}\right) .
$$

Now $A$ is almost in the required form and the nonzero entries in the first column can be easily removed by multiplication by elementary matrices.

Lemma 3.9. The natural maps

$$
\mathrm{E}_{l}\left(D_{\rho_{1}}(G, K)\right) \rightarrow \mathrm{E}_{l}\left(D_{\rho_{2}}(G, K)\right)
$$

have dense image for any $p^{-1}<\rho_{2}<\rho_{1}<1$ and positive integer $l$.

Proof. Since any element in $\mathrm{E}_{l}\left(D_{\rho_{2}}(G, K)\right)$ is the product of finitely many elementary matrices the result follows from the density of the image of the map $D_{\rho_{1}}(G, K) \rightarrow D_{\rho_{2}}(G, K)$.

The main result of this section is the following generalization of Theorem 1 in $V$. of $[\mathrm{G}]$.

Theorem 3.10. Let $G$ be a compact locally $\mathbb{Q}_{p}$-analytic group of dimension $d$. All the projective objects in the category of coadmissible modules over $D(G, K)$ are finitely generated. In particular any projective object is a projective module over $D(G, K)$ and the category does not have enough projectives. 
Remark. Whenever $G$ is uniform the rank of a projective coadmissible $D(G, K)$-module $P$ is defined by the rank of $D_{\rho}(G, K) \otimes P$. This is clearly independent of $\rho$.

Proof. It suffices to prove the statement when $G$ is uniform as any compact $p$-adic Lie group has a finite index uniform subgroup.

Now assume that $G$ is uniform and let $P$ be a projective object in the category of coadmissible $D(G, K)$-modules. We claim first that for any $\rho$ in $p^{\mathbb{Q}}$ with $1 / p<\rho<1$ the $D_{\rho}(G, K)$-module $P_{\rho}:=D_{\rho}(G, K) \otimes_{D(G, K)} P$ is also projective (and finitely generated since $P$ is coadmissible). Let $m_{1}, \ldots, m_{l}$ be elements in $P$ such that $1 \otimes m_{1}, \ldots, 1 \otimes m_{l}$ generate $P_{\rho}=D_{\rho}(G, K) \otimes P$. Take the map

$$
\begin{aligned}
\varphi: D(G, K)^{l} & \rightarrow P \\
e_{j} & \mapsto m_{j}
\end{aligned}
$$

where $e_{j}$ is the $j$ th standard basis vector of $D(G, K)^{l}$. Since $P$ is a projective object we obtain an exact sequence

$$
0 \rightarrow \operatorname{Hom}(P, \operatorname{Ker}(\varphi)) \rightarrow \operatorname{Hom}\left(P, D(G, K)^{l}\right) \rightarrow \operatorname{Hom}(P, P) \rightarrow \operatorname{Hom}(P, \operatorname{Coker}(\varphi)) \rightarrow 0
$$

of coadmissible modules. By Lemma 8.4 in ST5 the following sequence is also exact for any $p^{-1}<\rho<1$ in $p^{\mathbb{Q}}$.

$$
\begin{array}{r}
0 \rightarrow \operatorname{Hom}_{D_{\rho}(G, K)}\left(P_{\rho}, \operatorname{Ker}(\varphi)_{\rho}\right) \rightarrow \operatorname{Hom}_{D_{\rho}(G, K)}\left(P_{\rho}, D_{\rho}(G, K)^{l}\right) \rightarrow \\
\rightarrow \operatorname{Hom}_{D_{\rho}(G, K)}\left(P_{\rho}, P_{\rho}\right) \rightarrow \operatorname{Hom}_{D_{\rho}(G, K)}\left(P_{\rho}, \operatorname{Coker}(\varphi)_{\rho}\right) \rightarrow 0
\end{array}
$$

On the other hand $\operatorname{Coker}(\varphi)_{\rho}=0$ as $1 \otimes m_{1}, \ldots, 1 \otimes m_{l}$ generate $P_{\rho}$. So the map

$$
\operatorname{Hom}\left(P_{\rho}, D_{\rho}(G, K)^{l}\right) \rightarrow \operatorname{Hom}\left(P_{\rho}, P_{\rho}\right)
$$

is surjective which means that $P_{\rho}$ is a direct summand of $D_{\rho}(G, K)^{l}$ and hence projective.

By Corollary 3.7 the $D_{\rho}(G, K)$-module $P_{\rho} \oplus D_{\rho}(G, K)^{\max (\operatorname{rk}(P), d+2)-\operatorname{rk}(P)}$ is free of rank $\max (\operatorname{rk}(P), d+2)$. So we have

$$
\begin{aligned}
& P \oplus D(G, K)^{\max (\operatorname{rk}(P), d+2)-\operatorname{rk}(P)} \cong{\underset{\iota}{\lim _{\rho}}}_{\rho} P_{\rho} \underset{\underbrace{}_{\rho}}{\lim } D_{\rho}(G, K)^{\max (\operatorname{rk}(P), d+2)-\operatorname{rk}(P)} \\
& \cong \lim _{\rho}\left(P_{\rho} \oplus D_{\rho}(G, K)^{\max (\operatorname{rk}(P), d+2)-\mathrm{rk}(P)}\right) \\
& \cong \lim _{\rho} D_{\rho}(G, K)^{\max (\mathrm{rk}(P), d+2)} \text {. }
\end{aligned}
$$

Note that the connecting maps in $\lim _{\coprod_{\rho}} D_{\rho}(G, K)^{\max (\mathrm{rk}(P), d+2)}$ may not map the standard basis vectors to each other. This is the reason why we need Lemma 3.8. We are going to show that $D(G, K)^{m+d+2}$ surjects onto $P \oplus D(G, K)^{m-\operatorname{rk}(P)}$ where $m=\max (\operatorname{rk}(P), d+2)$. This will show that $P$ is finitely generated. Let $E_{\rho}$ be the set of surjections from $D_{\rho}(G, K)^{m+d+2}$ to $D(G, K)_{\rho}^{m}$. The sets $E_{\rho}$ form a projective system with continuous connecting maps and it suffices to show that the projective limit of this system is nonempty. By Lemma 3.8 we have a transitive and continuous action of $\mathrm{E}_{m+d+2}\left(D_{\rho}(H, K)\right)$ on $E_{\rho}$ for each $p^{-1} \leq \rho<1$ in $p^{\mathbb{Q}}$. Applying Lemma 3.9 with $l=m+d+2$ we obtain that the image of $E_{\rho_{1}}$ in $E_{\rho_{2}}$ is also dense. 
Therefore the projective system $\left(E_{\rho}\right)_{\rho}$ satisfies the Mittag-Leffler condition so its projective limit is nonempty.

Once we know that $P$ is finitely generated, it is easy to see that $P$ is a projective module over $D(G, K)$ because it is the direct summand of a free module (of finite rank) as the finitely generated free modules over $D(G, K)$ are coadmissible. The last statement follows from the fact that there are coadmissible modules over $D(G, K)$ which are not finitely generated.

Remarks. 1. The conversion of Proposition 3.10 is also true. If $P$ is a finitely generated projective module over $D(G, K)$ then it is of course finitely presented and so coadmissible by Corollary $3.4 v$ in [ST5.

2. It would be interesting to have a generalization of Proposition 3.10 to any noncommutative Fréchet-Stein algebra. However, general noetherian Banach algebras might have infinite Krull dimension. Moreover, even in the case of commutative locally $L$ analytic groups where $L \supsetneq \mathbb{Q}_{p}$ the Grothendieck group $K_{0}\left(D_{\rho}(G, K)\right)$ is more complicated. For example when $o_{L}$ is the additive group of the ring of integers in $L$ we have $K_{0}\left(D_{\rho}\left(o_{L}, K\right)\right) \neq \mathbb{Z}($ see $[\mathrm{S} 2])$.

\section{Fréchet-Stein structure}

Let $G$ be a uniform pro- $p$ group. In this section we are going to prove that the topological $K$-algebras $D_{[\rho, 1)}(G, K)$ constructed in the Appendix are Fréchet-Stein.

Let $p^{-1}<\rho_{1}<\rho_{3}<\rho_{2}<1$ be real numbers in $p^{\mathbb{Q}}$. Let $D_{\left[\rho_{1}, \rho_{2}\right)^{b d}}(G, K)$ and $D_{\left(\rho_{1}, \rho_{2}\right]^{b d}}(G, K)$ be the subspace of $D_{\left[\rho_{1}, \rho_{3}\right]}(G, K)$ and $D_{\left[\rho_{3}, \rho_{2}\right]}(G, K)$, respectively, consisting of those Laurent series that are bounded in the norm $\|\cdot\|_{\rho_{1}, \rho_{2}}:=\max \left(\|\cdot\|_{\rho_{1}},\|\cdot\|_{\rho_{2}}\right)$. This definition is clearly independent of the choice of $\rho_{3}$. These are a priori Banach spaces, however, since the norm $\|\cdot\|_{\rho_{1}, \rho_{2}}$ is submultiplicative on monomials they form subalgebras of $D_{\left[\rho_{1}, \rho_{3}\right]}(G, K)$ and $D_{\left[\rho_{3}, \rho_{2}\right]}(G, K)$, respectively. Moreover, this norm induces a filtration on each algebra given by

$$
\begin{aligned}
& F_{\rho_{1}, \rho_{2}}^{s} D_{*}(G, K):=\left\{x \in D_{*}(G, K):\|x\|_{\rho_{1}, \rho_{2}} \leq p^{-s}\right\}, \\
& F_{\rho_{1}, \rho_{2}}^{s+} D_{*}(G, K):=\left\{x \in D_{*}(G, K):\|x\|_{\rho_{1}, \rho_{2}}<p^{-s}\right\}
\end{aligned}
$$

where $*$ denotes either $\left[\rho_{1}, \rho_{2}\right],\left(\rho_{1}, \rho_{2}\right]^{b d}$, or $\left[\rho_{1}, \rho_{2}\right)^{b d}$.

Proposition 4.1. If $\rho_{1}$ and $\rho_{2}$ are both in $p^{\mathbb{Q}}$ then the above algebras $D_{*}(G, K)$ are all noetherian.

Proof. We may extend scalars without loss of generality to a finite extension of $K$. So we assume that both $\rho_{1}$ and $\rho_{2}$ are integral powers of the absolute value of the uniformizer of $K$. At first we prove the statement for $D_{\left[\rho_{1}, \rho_{2}\right]}(G, K)$. The other cases are dealt with in a similar way. The underlying space of the graded $\operatorname{ring} \operatorname{gr}_{\rho_{1}, \rho_{2}} D_{\left[\rho_{1}, \rho_{2}\right]}(G, K)$ is the set of Laurent polynomials in variables $X_{1}, \ldots, X_{d}$ over the graded ring gr $K=k\left[X_{0}, X_{0}^{-1}\right]$ of $K$. Here $X_{0}$ is the principal symbol of the uniformizer of the field $K$ and $X_{i}=\sigma\left(b_{i}\right) / X_{0}^{s}$ for each $1 \leq i \leq d$ where $\rho_{2}$ is the $s$ th power of the absolute value of the unformizer of $K$. The gr $K$-module structure is clear the question is only the multiplication of monomials $\mathbf{X}^{\alpha}=X_{1}^{\alpha_{1}} \cdots X_{d}^{\alpha_{d}}$. 
However, this is non-standard, the product of two monomials $\mathbf{X}^{\alpha}$ and $\mathbf{X}^{\beta}$ is defined for any $\alpha$ and $\beta$ in $\mathbb{Z}^{d}$ by

$$
\mathbf{X}^{\alpha} \mathbf{X}^{\beta}= \begin{cases}0 & \text { if }|\alpha|<0 \text { and }|\beta|>0 \text { or if }|\alpha|>0 \text { and }|\beta|<0 \\ \mathbf{X}^{\alpha+\beta} & \text { otherwise. }\end{cases}
$$

Indeed, we have $\left\|\mathbf{b}^{\alpha} \mathbf{b}^{\beta}\right\|_{\rho_{1}, \rho_{2}}=\left\|\mathbf{b}^{\alpha}\right\|_{\rho_{1}, \rho_{2}}\left\|\mathbf{b}^{\beta}\right\|_{\rho_{1}, \rho_{2}}$ if and only if

$$
\begin{cases}\text { either } & \left\|\mathbf{b}^{\alpha}\right\|_{\rho_{1}, \rho_{2}}=\left\|\mathbf{b}^{\alpha}\right\|_{\rho_{1}} \text { and }\left\|\mathbf{b}^{\beta}\right\|_{\rho_{1}, \rho_{2}}=\left\|\mathbf{b}^{\beta}\right\|_{\rho_{1}} \\ \text { or } & \left\|\mathbf{b}^{\alpha}\right\|_{\rho_{1}, \rho_{2}}=\left\|\mathbf{b}^{\alpha}\right\|_{\rho_{2}} \text { and }\left\|\mathbf{b}^{\beta}\right\|_{\rho_{1}, \rho_{2}}=\left\|\mathbf{b}^{\beta}\right\|_{\rho_{2}} .\end{cases}
$$

Since $\rho_{1}<\rho_{2}$ the two norms of the term $\mathbf{b}^{\alpha}$ coincide if and only if the degree $|\alpha|$ of $\alpha$ is zero. So the condition (6) is equivalent to that at least one of $|\alpha|$ and $|\beta|$ is zero or they have the same sign and claim (5) follows.

Now the filtration on $D_{\left[\rho_{1}, \rho_{2}\right]}(G, K)$ is complete and indexed by the integer multiples of a rational number, so by Prop. I.7.1.2 in [LvO] it suffices to show that $\operatorname{gr}_{\rho_{1}, \rho_{2}} D_{\left[\rho_{1}, \rho_{2}\right]}(G, K)$ is noetherian. For this take an ideal $I$ in $\operatorname{gr}_{\rho_{1}, \rho_{2}} D_{\left[\rho_{1}, \rho_{2}\right]}(G, K)$. Let $\pi$ denote the projection of $\operatorname{gr}_{\rho_{1}, \rho_{2}} D_{\left[\rho_{1}, \rho_{2}\right]}(G, K)$ to the terms $\sum c_{\alpha} \mathbf{X}^{\alpha}$ with $c_{\alpha}$ in $k\left[X_{0}, X_{0}^{-1}\right]$ and $\alpha$ in $\mathbb{Z}^{d}$ with $|\alpha| \geq 0$. This projection $\pi$ is in fact a ring homomorphism onto the Laurent polynomial ring

$$
B:=k\left[X_{0}, X_{0}^{-1}, X_{1}, X_{2} X_{1}^{-1}, \ldots, X_{d} X_{1}^{-1}, X_{1} X_{2}^{-1}, \ldots, X_{1} X_{d}^{-1}\right]
$$

which is noetherian. So $\pi(I)$ is finitely generated. On the other hand $\operatorname{Ker}(\pi) \cap I$ is also an ideal in the Laurent polynomial ring

$$
k\left[X_{0}, X_{0}^{-1}, X_{1}^{-1}, X_{2} X_{1}^{-1}, \ldots, X_{d} X_{1}^{-1}, X_{1} X_{2}^{-1}, \ldots, X_{1} X_{d}^{-1}\right]
$$

which is viewed as a subring of $\operatorname{gr}_{\rho_{1}, \rho_{2}} D_{\left[\rho_{1}, \rho_{2}\right]}(G, K)$. This is also noetherian and therefore $\operatorname{Ker}(\pi) \cap I$ is also finitely generated. Putting together these generators with (any) lifts of the generators of $\pi(I)$ we get a finite generating system of $I$ and the result follows for $D_{\left[\rho_{1}, \rho_{2}\right]}(G, K)$.

The proof of the statement for the rings $D_{\left(\rho_{1}, \rho_{2}\right]^{b d}}(G, K)$ and $D_{\left[\rho_{1}, \rho_{2}\right)^{b d}}(G, K)$ is similar. We only show it for $D_{\left[\rho_{1}, \rho_{2}\right)^{b d}}(G, K)$ and leave the other case to the reader. The underlying space of the graded ring $\operatorname{gr}_{\rho_{1}, \rho_{2}}^{\circ} D_{\left[\rho_{1}, \rho_{2}\right)^{b d}}(G, K)$ is the Laurent polynomial ring $C\left[X_{0}, X_{0}^{-1}\right]$ over the ring

$$
C:=k\left[X_{2} X_{1}^{-1}, \ldots, X_{d} X_{1}^{-1}, X_{1} X_{2}^{-1}, \ldots, X_{1} X_{d}^{-1}\right]\left(\left(X_{1}\right)\right) .
$$

The multiplication is still given by (5). Hence it remains to show that the ring

$$
C_{0}:=k\left[X_{2} X_{1}^{-1}, \ldots, X_{d} X_{1}^{-1}, X_{1} X_{2}^{-1}, \ldots, X_{1} X_{d}^{-1}\right]\left[\left[X_{1}\right]\right]
$$

is noetherian as this is the 'positive part' of $\operatorname{gr}_{\rho_{1}, \rho_{2}} D_{\left[\rho_{1}, \rho_{2}\right)^{b d}}(G, K)$. However, $C_{0}$ is also completely filtered by the lowest degree and the corresponding graded ring is the Laurent polynomial ring

$$
k\left[X_{1}, X_{2} X_{1}^{-1}, \ldots, X_{d} X_{1}^{-1}, X_{1} X_{2}^{-1}, \ldots, X_{1} X_{d}^{-1}\right]
$$

which is noetherian. The result follows again by applying Proposition I.7.1.2 of [LvO] twice. 
Lemma 4.2. The ring $C_{0}\left[X_{0}, X_{0}^{-1}\right]$ is flat over the ring $B$. (For the definition of $B$ and $C_{0}$ see (7) and (8), respectively.)

Proof. The ring of formal power series (in the variable $X_{1}$ ) over any ring $R$ is well-known to be flat over the ring of polynomials. Moreover, if the $\operatorname{ring} R_{2}$ is flat over the $\operatorname{ring} R_{1}$ then so is the Laurent polynomial ring $R_{2}\left[X_{0}, X_{0}^{-1}\right]$ over $R_{1}\left[X_{0}, X_{0}^{-1}\right]$. Here we use these statements with

$$
\begin{aligned}
R & :=k\left[X_{2} X_{1}^{-1}, \ldots, X_{d} X_{1}^{-1}, X_{1} X_{2}^{-1}, \ldots, X_{1} X_{d}^{-1}\right] \\
R_{1} & :=k\left[X_{1}, X_{2} X_{1}^{-1}, \ldots, X_{d} X_{1}^{-1}, X_{1} X_{2}^{-1}, \ldots, X_{1} X_{d}^{-1}\right] \\
R_{2} & :=C_{0}
\end{aligned}
$$

and obtain the statement of the Lemma.

Now we can state our main Theorem in this section which is a slight generalization of Theorem 4.9 of [ST5].

Theorem 4.3. The topological $K$-algebra $D_{[\rho, 1)}(G, K)$ is a Fréchet-Stein $K$-algebra for any $p^{-1}<\rho<1$ in $p^{\mathbb{Q}}$.

Proof. We certainly have

$$
D_{[\rho, 1)}(G, K)=\lim _{\rho_{2} \rightarrow 1} D_{\left[\rho, \rho_{2}\right]}(G, K)
$$

is a limit of noetherian Banach algebras. So it suffices to show that whenever $\rho<\rho_{2}<\rho_{3}<1$ are all in $p^{\mathbb{Q}}$ then $D_{\left[\rho, \rho_{2}\right]}(G, K)$ is flat as a (left or right) $D_{\left[\rho, \rho_{3}\right]}(G, K)$-module. As in the proof of Theorem 4.9 of [ST5] we do this in two steps. We show that both of the maps

$$
\begin{aligned}
D_{\left[\rho, \rho_{3}\right]}(G, K) & \hookrightarrow D_{\left[\rho, \rho_{3}\right)^{b d}}(G, K) \text { and } \\
D_{\left[\rho, \rho_{3}\right)^{b d}}(G, K) & \hookrightarrow D_{\left[\rho, \rho_{2}\right]}(G, K)
\end{aligned}
$$

are flat and then so is their composite. Both of the statements can be checked over a finite extension of $K$ as the tensor product with a finite extension is faithfully flat.

We begin with showing that the map (9) is flat. By Proposition 1.2 in [ST5] (see also [LvO]) it suffices to verify that the map of graded rings

$$
\operatorname{gr}_{\rho, \rho_{3}}^{\cdot} D_{\left[\rho, \rho_{3}\right]}(G, K) \hookrightarrow \operatorname{gr}_{\rho, \rho_{3}}^{\cdot} D_{\left[\rho, \rho_{3}\right)^{b d}}(G, K)
$$

is flat. However, the functors

$$
\operatorname{gr}_{\rho, \rho_{3}}^{\cdot} D_{\left[\rho, \rho_{3}\right)}(G, K) \otimes_{\operatorname{gr}_{\rho, \rho_{3}}^{*} D_{\left[\rho, \rho_{3}\right]}(G, K)} \cdot \quad \text { and } \quad C_{0}\left[X_{0}, X_{0}^{-1}\right] \otimes_{B} \cdot
$$

coincide on $\operatorname{gr}_{\rho, \rho_{3}} D_{\left[\rho, \rho_{3}\right]}(G, K)$-modules and the assertion follows from Lemma 4.2.

Now we prove the flatness of (10). For this we are going to use the filtration $F_{\rho, \rho_{2}}^{s}$ induced by the norm $\|\cdot\|_{\rho, \rho_{2}}$ on both $D_{\left[\rho, \rho_{3}\right)^{b d}}(G, K)$ and $D_{\left[\rho, \rho_{2}\right]}(G, K)$. This filtration is not complete on $D_{\left[\rho, \rho_{3}\right)^{b d}}(G, K)$, however, it is complete on its subring $F_{\rho, \rho_{3}}^{0} D_{\left[\rho, \rho_{3}\right)^{b d}}(G, K)$. It suffices to prove the flatness of $D_{\left[\rho, \rho_{2}\right]}(G, K)$ over $F_{\rho, \rho_{3}}^{0} D_{\left[\rho, \rho_{3}\right)^{b d}}(G, K)$ as we have

$$
D_{\left[\rho, \rho_{3}\right)^{b d}}(G, K)=\mathbb{Q}_{p} \otimes_{\mathbb{Z}_{p}} F_{\rho, \rho_{3}}^{0} D_{\left[\rho, \rho_{3}\right)^{b d}}(G, K) .
$$


As the filtration is complete on both sides we are reduced to show by Proposition 1.2 of [ST5] that the map of graded rings

$$
\operatorname{gr}_{\rho, \rho_{2}}^{\cdot} F_{\rho, \rho_{3}}^{0} D_{\left[\rho, \rho_{3}\right)^{b d}}(G, K) \hookrightarrow \operatorname{gr}_{\rho, \rho_{2}}^{\cdot} D_{\left[\rho, \rho_{2}\right]}(G, K)
$$

is flat. This is clear as the right hand side of (11) is the localization of the left hand side at $X_{0}$.

\section{Topologies and self-duality}

Recall that the classical Robba $\operatorname{ring} \mathcal{R}=\mathcal{R}\left(\mathbb{Z}_{p}, K\right)$ can be identified with the ring of formal Laurent-series over $K$ that are convergent in some open annulus with outer radius 1 . $\mathcal{R}$ is a self-dual topological $K$-algebra with respect to the perfect pairing [GiRo]

$$
\begin{aligned}
\mathcal{R} \times \mathcal{R} & \rightarrow K \\
(x, y) & \mapsto \text { "constant term of the power series } x(T) y(T)^{\prime \prime} .
\end{aligned}
$$

In this section we are going to show that the Robba ring for more general uniform pro- $p$ groups defined in the Appendix is also self-dual in an appropriate topology and pairing.

\subsection{The weak topology}

An immediate consequence of Proposition A.24 in the Appendix is that $\mathcal{R}(G, K)$ is also a topological $K$-algebra with the inductive limit topology. However, we need to introduce yet another topology on each $D_{[\rho, 1)}(G, K)$ and on $\mathcal{R}(G, K)$ so that they all become self-dual. Let $R$ denote any of the rings $D_{[\rho, 1)}(G, K)$ or $\mathcal{R}(G, K)$. Then we define the "weak topology" on $R$ as the coarsest locally convex topology such that for any (fixed) $x_{0}$ in $R$ the map

$$
\begin{aligned}
R & \rightarrow K \\
x & \mapsto\left(x_{0}, x\right):=\text { "the constant term of } x_{0} x^{\prime \prime}
\end{aligned}
$$

is continuous. We shall see later on that these topologies are different from the usual (Fréchet/inductive limit) topology on each $R$, the weak topology is strictly coarser. Let us first remark that the multiplication on $R$ is separately continuous in the weak topology. Now we need the following lemmata.

Lemma 5.1. For any index $\alpha$ in $\mathbb{Z}^{d}$ the following statements hold.

(i) For any index $\beta \neq-\alpha$ we have $\left|\left(\mathbf{b}^{\alpha}, \mathbf{b}^{\beta}\right)\right| \leq \min \left(1, p^{-|\alpha|-|\beta|}\right)$. Moreover, if we assume further that $|\alpha|+|\beta|=0$ then we have $\left|\left(\mathbf{b}^{\alpha}, \mathbf{b}^{\beta}\right)\right| \leq p^{-1}$.

(ii) For any given real number $\varepsilon>0$ and integer $m$ there are only finitely many indices $\beta$ in $\mathbb{Z}^{d}$ such that $|\beta|=m$ and $\left|\left(\mathbf{b}^{\alpha}, \mathbf{b}^{\beta}\right)\right|>\varepsilon$.

Proof. The first assertion follows immediately from Lemma A.25 in the Appendix.

For $(i i)$ we are going to prove the following quantitative estimate. Put

$$
N:=\max \left(\alpha_{1}+\beta_{1}, \ldots, \alpha_{d}+\beta_{d}\right) .
$$


Then we have

$$
\left|\left(\mathbf{b}^{\alpha}, \mathbf{b}^{\beta}\right)\right| \leq p^{-\frac{|\alpha|+|\beta|+N}{2}} .
$$

Indeed, the result follows from this noting that for any fixed degree and fixed maximum of the coordinates there are only finitely many $\beta$.

Now we fix $\alpha$ and $\beta$ in order to prove (13). We may assume without loss of generality for the moment that $K=\mathbb{Q}_{p}$ as the product $\mathbf{b}^{\alpha} \mathbf{b}^{\beta}$ is already defined over $\mathbb{Q}_{p}$ (and even over $\mathbb{Z}_{p}$ ). Now we analyze further the Laurent-series expansion of $\mathbf{b}^{\alpha} \mathbf{b}^{\beta}$ by giving an (infinite) algorithm computing it. The algorithm is the following:

Before the $n$th step of the algorithm we have a formal sum

$$
\sum_{i=1}^{\infty} c_{n, i} w_{n, i}
$$

where $c_{n, i}$ is in $\mathbb{Z}_{p}$ and $w_{n, i}$ is a word in the free group $F\left(b_{1}, \ldots, b_{d}\right)$ on the generators $b_{1}, \ldots, b_{d}$ such that the sum satisfies the convergence condition

$$
\lim _{i \rightarrow \infty}\left\|c_{n, i} w_{n, i}\right\|_{p^{-1 / 2}}=0
$$

Since the norm $\|\cdot\|_{p^{-1 / 2}}$ is multiplicative on $D_{\left[p^{-1 / 2}, 1\right)}(G, K)$, we have

$$
\left\|c_{n, i} w_{n, i}\right\|_{p^{-1 / 2}}=\left|c_{n, i}\right| p^{-\frac{\left|w_{n, i}\right|}{2}}
$$

where $\left|w_{n, i}\right|$ is the image of $w_{n, i}$ under the homomorphism from $F\left(b_{1}, \ldots, b_{d}\right)$ to $\mathbb{Z}$ sending all the generators to 1 . We also assume for the sake of simplicity that the terms $c_{n, i} w_{n, i}$ are in decreasing order with respect to the norm $\|\cdot\|_{p^{-1 / 2}}$. Note that granted the convergence criterion (15) the above sum defines an element in the ring $D_{\left[p^{-1 / 2}, 1\right)}(G, K)$, however it is not in the canonical form. Indeed, the sum (14) is also convergent in any other norm $\|\cdot\|_{\rho}$ with $p^{-1 / 2} \leq \rho<1$ because the coefficients are in $\mathbb{Z}_{p}$. We call a word $w$ in the free group $F\left(b_{1}, \ldots, b_{d}\right)$ bad if it is not of the form

$$
w=b_{1}^{\alpha_{1}} b_{2}^{\alpha_{2}} \cdots b_{d}^{\alpha_{d}} .
$$

Before the first step we have $w_{1,1}=\mathbf{b}^{\alpha} \mathbf{b}^{\beta}, c_{1,1}=1$, and all the other terms are 0 in the sum. Now we describe the $n$th step of the algorithm producing the sum $\sum_{i=1}^{\infty} c_{n+1, i} w_{n+1, i}$ from $\sum_{i=1}^{\infty} c_{n, i} w_{n, i}$ such that in $D_{\left[p^{-1 / 2}, 1\right)}\left(G, \mathbb{Q}_{p}\right)$ they converge to the same element. Let $s_{n}$ denote the smallest positive integer such that $w_{n, s_{n}}$ is a bad word. If there is no such an integer then the algorithm terminates and our sum defines an element of $D_{\left[p^{-1 / 2}, 1\right)}\left(G, \mathbb{Q}_{p}\right)$ in the canonical form (and in fact of $D_{[\rho, 1)}\left(G, \mathbb{Q}_{p}\right)$ for any $p^{-1}<\rho<1$, but we do not need this). We are going to replace $w_{n, s_{n}}$ by an infinite sum of the form

$$
w_{0}+\sum_{i=1}^{\infty} c_{i} w_{i}
$$

such that we have

$$
\begin{aligned}
w_{n, s_{n}} & =w_{0}+\sum_{i=1}^{\infty} c_{i} w_{i} \text { in } D_{\left[p^{-1 / 2}, 1\right)}\left(G, \mathbb{Q}_{p}\right) \\
\left\|w_{n, s_{n}}\right\|_{p^{-1 / 2}} & =\left\|w_{0}\right\|_{p^{-1 / 2}}>\left\|c_{i} w_{i}\right\|_{p^{-1 / 2}} \text { for any } i>0 ; \quad \text { and } \\
\lim _{i \rightarrow \infty}\left\|c_{i} w_{i}\right\|_{p^{-1 / 2}} & =0 .
\end{aligned}
$$


For this we write $w_{n, s_{n}}$ in the form

$$
b_{x_{1}}^{e_{1}} b_{x_{2}}^{e_{2}} \ldots b_{x_{z}}^{e_{z}}
$$

where $1 \leq x_{j} \leq d$ and $e_{j}$ are +1 or -1 for each $1 \leq j \leq z$. Now let $t$ be the smallest positive integer such that $x_{t}>x_{t+1}$. There exists such a $t$ since the word $w_{n, s_{n}}$ is bad. We would like to swap $b_{x_{t}}^{e_{t}}$ and $b_{x_{t+1}}^{e_{t+1}}$ in the word $w$ so that the word becomes 'less bad'. To do this we need the following identities that hold in any associative ring in which $b_{x_{t}}$ and $b_{x_{t+1}}$ are invertible.

$$
\begin{aligned}
b_{x_{t}} b_{x_{t+1}} & =b_{x_{t+1}} b_{x_{t}}+\left(b_{x_{t}} b_{x_{t+1}}-b_{x_{t+1}} b_{x_{t}}\right), \\
b_{x_{t}}^{-1} b_{x_{t+1}} & =b_{x_{t+1}} b_{x_{t}}^{-1}+b_{x_{t}}^{-1}\left(b_{x_{t+1}} b_{x_{t}}-b_{x_{t}} b_{x_{t+1}}\right) b_{x_{t}}^{-1}, \\
b_{x_{t}} b_{x_{t+1}}^{-1} & =b_{x_{t+1}}^{-1} b_{x_{t}}+b_{x_{t+1}}^{-1}\left(b_{x_{t+1}} b_{x_{t}}-b_{x_{t}} b_{x_{t+1}}\right) b_{x_{t+1}}^{-1}, \text { and } \\
b_{x_{t}}^{-1} b_{x_{t+1}}^{-1} & =b_{x_{t+1}}^{-1} b_{x_{t}}^{-1}+b_{x_{t}}^{-1} b_{x_{t+1}}^{-1}\left(b_{x_{t}} b_{x_{t+1}}-b_{x_{t+1}} b_{x_{t}}\right) b_{x_{t+1}}^{-1} b_{x_{t}}^{-1} .
\end{aligned}
$$

On the other hand $b_{x_{t}} b_{x_{t+1}}-b_{x_{t+1}} b_{x_{t}}$ has a canonical expansion $\sum_{\gamma} a_{\gamma} \mathbf{b}^{\gamma}$ in the distribution algebra $D(G, K)$ with $a_{\gamma}$ in $\mathbb{Z}_{p}$. So by collecting all the identities in (19) and plugging in the canonical expansion of $b_{x_{t}} b_{x_{t+1}}-b_{x_{t+1}} b_{x_{t}}$ we obtain

$$
b_{x_{t}}^{e_{t}} b_{x_{t+1}}^{e_{t+1}}=b_{x_{t+1}}^{e_{t+1}} b_{x_{t}}^{e_{t}}+\sum_{\gamma \in \mathbb{N}_{0}^{d}} a_{\gamma} e_{t} e_{t+1} b_{x_{t}{ }^{2}}^{\frac{e_{t}-1}{x_{t+1}}} b_{x_{t+1}-1}^{e^{2}} \mathbf{b}^{\gamma} b_{x_{t+1}}^{\frac{e_{t+1}-1}{t_{2}}} b_{x_{t}{ }^{2}}^{\frac{e_{t}-1}{2}}
$$

in $D_{\left[p^{-1 / 2}, 1\right)}(G, K)$. Now we multiply both sides of the equations (20) formally by $b_{x_{1}}^{e_{1}} \cdots b_{x_{t-1}}^{e_{t-1}}$ from the left and by $b_{x_{t+2}}^{e_{t+2}} \cdots b_{x_{z}}^{e_{z}}$ from the right and get an equation of the form (16) with

$$
\begin{aligned}
w_{0} & :=b_{x_{1}}^{e_{1}} \cdots b_{x_{t-1}}^{e_{t-1}} b_{x_{t+1}}^{e_{t+1}} b_{x_{t}}^{e_{t}} b_{x_{t+2}}^{e_{t+2}} \cdots b_{x_{z}}^{e_{z}} \quad \text { and } \\
c_{i} & :=a_{\gamma_{i}} e_{t} e_{t+1} \\
w_{i} & :=b_{x_{1}}^{e_{1}} \cdots b_{x_{t-1}}^{e_{t-1}} b_{x_{t}}^{\frac{e_{t-1}}{2}} \frac{e_{x_{t+1}}}{e_{t+1}-1} \mathbf{b}^{\gamma_{i}} \frac{e_{t+1}-1}{x_{x_{t+1}}^{2}} b_{x_{t}}^{\frac{e_{t}-1}{2}} b_{x_{t+2}}^{e_{t+2}} \cdots b_{x_{z}}^{e_{z}}
\end{aligned}
$$

for an arbitrary (but fixed) ordering $\left(\gamma_{i}\right)_{i \geq 1}$ of the set $\mathbb{N}_{0}^{d}$. The properties (17) follow from Proposition A.21 and the convergence of the expansion of $b_{x_{t}} b_{x_{t+1}}-b_{x_{t+1}} b_{x_{t}}$.

We define $\sum_{i=1}^{\infty} c_{n+1, i} w_{n+1, i}$ by rearranging the sum

$$
\sum_{i=1}^{s_{n}-1} c_{n, i} w_{n, i}+c_{n, s_{n}} w_{0}+\sum_{i=1}^{\infty} c_{n, s_{n}} c_{i} w_{i}+\sum_{i=s_{n}+1}^{\infty} c_{n, i} w_{n, i}
$$

in norm decreasing order. Moreover, we may assume that $c_{n+1, i} w_{n+1, i}=c_{n, i} w_{n, i}$ for any $1 \leq i<s_{n}$ and $c_{n+1, s_{n}} w_{n+1, s_{n}}=c_{n, s_{n}} w_{0}$ as these terms are already in norm decreasing order. The description of the $n$th step of the algorithm ends here.

We claim that the formal sum

$$
\sum_{i=1}^{\infty} c_{\infty, i} w_{\infty, i}:=\sum_{i=1}^{\infty} \lim _{n \rightarrow \infty} c_{n, i} w_{n, i}
$$

makes sense and it equals the Laurent series expansion of $\mathbf{b}^{\alpha} \mathbf{b}^{\beta}$ in $D_{\left[p^{-1 / 2}, 1\right)}(G, K)$ (and hence in any of the rings $D_{[\rho, 1)}(G, K)$ ). By construction one of the following could happen after the $n$th step: 
1. $w_{0}$ is bad, $s_{n}=s_{n+1}$, and $w_{n+1, s_{n+1}}=w_{0}$.

2. $s_{n+1}>s_{n}$.

The first case can only happen finitely many times in a line. Indeed, if we define

$$
D\left(w_{s_{n}}\right):=\text { "the number of pairs } 1 \leq i<j \leq z \text { such that } x_{i}>x_{j} \text { " }
$$

then $D\left(w_{n, s_{n}}\right)>D\left(w_{n+1, s_{n+1}}\right)$ in the first case. However, $D\left(w_{n+1, s_{n+1}}\right)=0$ is equivalent to that $w_{n+1, s_{n+1}}$ is not a bad word. It follows that the second case appears infinitely many times, in other words $s_{n}$ tends to infinity. This shows that $\lim _{n \rightarrow \infty} c_{n, i} w_{n, i}$ exists for any $i$ since $c_{n, i} w_{n, i}=c_{n+1, i} w_{n+1, i}$ whenever $i<s_{n}$. Moreover, in the sum $\sum_{i} c_{n, i} w_{n, i}$ for any fixed $n$ there are only finitely many terms with the same norm. On the other hand, the new terms $c_{n, s_{n}} c_{i} w_{i}$ satisfy

$$
\left\|c_{n, s_{n}} c_{i} w_{i}\right\|_{p^{-1 / 2}}<\left\|c_{n, s_{n}} w_{n, s_{n}}\right\|_{p^{-1 / 2}}
$$

by (17). This implies that for any integer $n_{0}$ there is an $n>n_{0}$ such that $\left\|c_{n, s_{n}} w_{n, s_{n}}\right\|_{p^{-1 / 2}}>$ $\left\|c_{n+1, s_{n+1}} w_{n+1, s_{n+1}}\right\|_{p^{-1 / 2}}$ and hence the sum (23) is convergent in $D_{\left[p^{-1 / 2}, 1\right)}(G, K)$ because the range of the norm $\|\cdot\|_{p^{-1 / 2}}$ is discrete and the terms in each sum $\sum_{i=1}^{\infty} c_{n, i} w_{n, i}$ are in normdecreasing order. Moreover, none of the terms in this sum are bad (by construction) therefore this is the Laurent series expansion of $\mathbf{b}^{\alpha} \mathbf{b}^{\beta}$ since by construction we have the equality

$$
\sum_{i=1}^{\infty} c_{n, i} w_{n, i}=\sum_{i=1}^{\infty} c_{n+1, i} w_{n+1, i}
$$

in $D_{\left[p^{-1 / 2}, 1\right)}(G, K)$ for any positive integer $n$.

Now we show (13) by using the above algorithm. Put

$$
\begin{aligned}
f(c w) & :=\log _{p^{-1 / 2}}\|c w\|_{p^{-1 / 2}}+\max _{1 \leq j \leq d} m_{j}(w) \in \mathbb{Z}, \\
f_{n} & :=\inf _{i \geq 1} f\left(c_{n, i} w_{n, i}\right) \in \mathbb{Z} \cup\{-\infty\}, \quad \text { and } \\
f_{\infty} & :=\inf _{i \geq 1} f\left(c_{\infty, i} w_{\infty, i}\right) \in \mathbb{Z} \cup\{-\infty\}
\end{aligned}
$$

where $m_{j}$ is the homomorphism from the free group $F\left(b_{1}, \ldots, b_{d}\right)$ to $\mathbb{Z}$ sending $b_{j}$ to 1 and all the other generators to 0 (in other words $m_{j}(w)$ is the degree of $w$ in the variable $b_{j}$ ). We claim that for any $n$ we have $f_{n} \leq f_{n+1}$. In particular all these $f_{n}$ are finite. For this it suffices to show that we have

$$
f\left(c_{n, s_{n}} w_{n, s_{n}}\right)=f\left(c_{n, s_{n}} w_{0}\right) \leq f\left(c_{n, s_{n}} c_{i} w_{i}\right) .
$$

as the new terms in $\sum_{i=1}^{\infty} c_{n+1, i} w_{n+1, i}$ are exactly $c_{n, s_{n}} w_{0}$ and $c_{n, s_{n}} c_{i} w_{i}(i \geq 1)$. The equality part of (24) is obvious as $m_{j}\left(w_{n, s_{n}}\right)=m_{j}\left(w_{0}\right)$ for any $j$ and

$$
\left\|c_{n, s_{n}} w_{n, s_{n}}\right\|_{p^{-1 / 2}}=\left\|c_{n, s_{n}} w_{0}\right\|_{p^{-1 / 2}} \quad \text { (see equations (18) and (21)). }
$$

The inequality follows from the facts that (see equations (17) and (22))

$$
\begin{array}{r}
\left\|c_{n, s_{n}} c_{i} w_{i}\right\|_{p^{-1 / 2}}<\left\|c_{n, s_{n}} w_{n, s_{n}}\right\|_{p^{-1 / 2}}, \\
m_{j}\left(w_{i}\right)=m_{j}\left(b_{x_{1}}^{e_{1}} \cdots b_{x_{t-1}}^{e_{t-1}} b_{x_{t}}^{\frac{e_{t}-1}{2}} b_{x_{t+1}}^{\frac{e_{t+1}-1}{2}} \mathbf{b}^{\gamma_{i}} b_{x_{t+1}}^{\frac{e_{t+1}-1}{2}} b_{x_{t}{ }^{2}}^{\frac{e^{-1}}{2}} b_{x_{t+2}}^{e_{t+2}} \cdots b_{x_{z}}^{e_{z}}\right) \geq m_{j}\left(w_{n, s_{n}}\right)-1
\end{array}
$$


(since $\gamma_{i}$ is in $\mathbb{N}_{0}^{d}$ ), and the range of $f$ is in $\mathbb{Z}$. We also obtain $f_{n} \leq f_{\infty}$ for any $n \geq 1$ by taking the limit. In particular we have

$$
\begin{aligned}
|\alpha|+|\beta|+N=f_{1} \leq f_{\infty} & \leq f\left(\left(\mathbf{b}^{\alpha}, \mathbf{b}^{\beta}\right)\right)=\log _{p^{-1 / 2}}\left|\left(\mathbf{b}^{\alpha}, \mathbf{b}^{\beta}\right)\right| \quad \text { whence } \\
p^{-\frac{|\alpha|+|\beta|+N}{2}} & \geq p^{-\frac{\left.f\left(\mathbf{b}^{\alpha}, \mathbf{b}^{\beta}\right)\right)}{2}}=\left|\left(\mathbf{b}^{\alpha}, \mathbf{b}^{\beta}\right)\right|
\end{aligned}
$$

and we are done.

Remark. The above algorithm computing the Laurent series expansion of $\mathbf{b}^{\alpha} \mathbf{b}^{\beta}$ can actually be used to define the rings $D_{[\rho, 1)}(G, K)$ (for $p^{-1}<\rho<1$ in $p^{\mathbb{Q}}$ ) by the underlying vector space of formal Laurent series together with the multiplication given by this algorithm. One, of course, has to check the associative and distributive laws. However, if we think of $D_{[\rho, 1)}(G, K)$ as a subring of the completion $Q_{\rho}(G, K)$ of the field of fractions $Q\left(D_{\rho}(G, K)\right)$ of the algebra $D_{\rho}(G, K)$ then we obtain immediately that $D_{[\rho, 1)}(G, K)$ satisfies the ring axioms. Indeed, $D_{\rho}(G, K)$ is a noetherian domain, so it has a field of fractions. Moreover, the norm $\|\cdot\|_{\rho}$ is multiplicative on $D_{\rho}(G, K)$, so it can be extended multiplicatively to the field $Q\left(D_{\rho}(G, K)\right)$ of fractions and we can take the completion $Q_{\rho}(G, K)$ with respect to $\|\cdot\|_{\rho}$. The Laurent series expansions of elements in $D_{[\rho, 1)}(G, K)$ will all be convergent in $Q_{\rho}(G, K)$, so $D_{[\rho, 1)}(G, K)$ is naturally a subring of $Q_{\rho}(G, K)$.

Lemma 5.2. For any index $\alpha$ in $\mathbb{Z}^{d}$ and any $p^{-1}<\rho<1$ there is an element $f^{(\alpha)}$ in $D_{[\rho, 1)}(G, K)$ such that

$$
\left(f^{(\alpha)}, \mathbf{b}^{\beta}\right)=\delta_{\alpha \beta}
$$

for all $\beta$ in $\mathbb{Z}^{d}$. Here $\delta_{\alpha \beta}$ denotes the Kronecker delta, ie. $\delta_{\alpha \beta}=1$ if $\alpha=\beta$ and 0 otherwise.

Proof. We may assume without loss of generality that $K=\mathbb{Q}_{p}$. We are going to give the power series expansion of $f^{(\alpha)}$ by approximating it in the ring $D_{\left(p^{-1}, 1\right)}(G, K)$. We start with the term $f_{0}^{(\alpha)}:=\mathbf{b}^{-\alpha}$. As the coefficients of $\mathbf{b}^{-\alpha} \mathbf{b}^{\beta}$ are in $\mathbb{Z}_{p}$ we have

$$
\left|\left(f_{0}^{(\alpha)}, \mathbf{b}^{\beta}\right)-\delta_{\alpha \beta}\right| \leq 1
$$

Let us denote by $C_{0}$ the set of $\gamma$ such that

$$
\left|\left(f_{0}^{(\alpha)}, \mathbf{b}^{\gamma}\right)-\delta_{\alpha \gamma}\right|=1
$$

Note that this set may well be nonempty and even infinite when $G$ is noncommutative. However, by Lemma $5.1(i)$ all these $\gamma$ have degree $|\gamma|<|\alpha|$, and by $(i i)$ for any fixed degree there are only finitely many of them. Hence we can remove

$$
\frac{\left(f_{0}^{(\alpha)}, \mathbf{b}^{\gamma}\right)}{\left(\mathbf{b}^{-\gamma}, \mathbf{b}^{\gamma}\right)} \mathbf{b}^{-\gamma}
$$

from $f_{0}^{(\alpha)}$ at first for all the $\gamma$ in $C_{0}$ with maximal degree. Since $\left(\mathbf{b}^{-\gamma}, \mathbf{b}^{\gamma}\right)$ is in $\mathbb{Z}_{p}^{\times}$the coefficient of (25) is in $\mathbb{Z}_{p}$. Note that other "bad" $\gamma$ may as well arise, but all of them have strictly smaller 
degree and for any fixed degree there are only finitely many of them. Henceforth we get a convergent Laurent series in $D_{[\rho, 1)}(G, K)$ (for any $p^{-1}<\rho<1$ )

$$
\begin{aligned}
f_{1}^{(\alpha)} & :=f_{0}^{(\alpha)}-\sum_{i=1}^{\infty} a_{\gamma_{1, i}} \mathbf{b}^{-\gamma_{1, i}} \quad \text { such that } \\
\left|\left(f_{1}^{(\alpha)}, \mathbf{b}^{\beta}\right)-\delta_{\alpha \beta}\right| & \leq p^{-1} \quad \text { for any } \beta \in \mathbb{Z}^{d} \quad \text { and } \\
\left\|f_{1}^{(\alpha)}-f_{0}^{(\alpha)}\right\|_{\rho} & \leq \rho\left\|f_{0}^{(\alpha)}\right\|_{\rho}
\end{aligned}
$$

since $\left|\gamma_{1, i}\right|<|\alpha|$ tends to $-\infty$ with $i \rightarrow \infty$. Here we choose recursively $\gamma_{1, i}$ with maximal degree so that

$$
\left|\left(f_{0}^{(\alpha)}-\sum_{j=1}^{i-1} a_{\gamma_{1, j}} \mathbf{b}^{-\gamma_{1, j}}, \mathbf{b}^{\gamma_{1, i}}\right)-\delta_{\alpha \gamma_{1, i}}\right|=1
$$

and put

$$
a_{\gamma_{1, i}}:=\frac{\left(f_{0}^{(\alpha)}-\sum_{j=1}^{i-1} a_{\gamma_{1, j}} \mathbf{b}^{-\gamma_{1, j}}, \mathbf{b}^{\gamma_{1, i}}\right)}{\left(\mathbf{b}^{-\gamma_{1, i}}, \mathbf{b}^{\gamma_{1, i}}\right)} \in \mathbb{Z}_{p} .
$$

Now we use the above method in order to modify $f_{n-1}^{(\alpha)}$ in the $n$th step $(n \geq 2)$ by a Laurent series with coefficients in $p^{n-1} \mathbb{Z}_{p}$ and minimal degree $-|\alpha|-n+1$, such that

$$
\begin{aligned}
\left|\left(f_{n}^{(\alpha)}, \mathbf{b}^{\beta}\right)-\delta_{\alpha \beta}\right| & \leq p^{-n} \text { and } \\
\left\|f_{n}^{(\alpha)}-f_{n-1}^{(\alpha)}\right\|_{\rho} & \leq \frac{1}{p^{n-1}} \rho^{-|\alpha|-n+1} .
\end{aligned}
$$

Indeed, we choose $\gamma_{n, i}$ with maximal degree so that

$$
\left|\left(f_{n-1}^{(\alpha)}-\sum_{j=1}^{i-1} a_{\gamma_{n, j}} \mathbf{b}^{-\gamma_{n, j}}, \mathbf{b}^{\gamma_{n, i}}\right)-\delta_{\alpha \gamma_{n, i}}\right|=p^{-n+1}
$$

and put

$$
\begin{aligned}
f_{n}^{(\alpha)} & :=f_{n-1}^{(\alpha)}-\sum_{i=1}^{\infty} a_{\gamma_{n, i}} \mathbf{b}^{-\gamma_{n, i}} \text { where } \\
a_{\gamma_{n, i}} & :=\frac{\left(f_{n-1}^{(\alpha)}-\sum_{j=1}^{i-1} a_{\gamma_{n, j}} \mathbf{b}^{-\gamma_{n, j}}, \mathbf{b}^{\gamma_{n, i}}\right)}{\left(\mathbf{b}^{-\gamma_{n, i}}, \mathbf{b}^{\gamma_{n, i}}\right)} \in p^{n-1} \mathbb{Z}_{p} .
\end{aligned}
$$

So by (27) the sequence $f_{n}^{(\alpha)}$ is Cauchy in each norm $\|\cdot\|_{\rho}$ and therefore defines an element

$$
f^{(\alpha)}:=\lim _{n \rightarrow \infty} f_{n}^{(\alpha)}
$$

in $D_{[\rho, 1)}(G, K)$ with the required property for any $\rho>p^{-1}$. Moreover, one has

$$
\left\|f^{(\alpha)}-\mathbf{b}^{-\alpha}\right\|_{\rho}<\left\|\mathbf{b}^{-\alpha}\right\|_{\rho}
$$

for any $p^{-1}<\rho<1$ by (26) and (27). Note that the difference between (26) and (27) for $n=1$ comes from the fact that we have a better estimate in Lemma $5.1(i)$ whenever $|\alpha|+|\beta|=0$. 
Lemma 5.3. The "Laurent polynomials" are dense in each $D_{[\rho, 1)}(G, K)$ (in both the Fréchet and the weak topologies). In particular they form a dense subset of $\mathcal{R}(G, K)$ in both the inductive limit topology and the weak topology.

Proof. The density in the Fréchet, resp. the inductive limit topology follows easily from the definition. On the other hand the maps (12) are continuous in the Fréchet, resp. the inductive limit topology. So any subset of $R$ that is open in the weak topology is also open in the Fréchet, resp. the inductive limit topology hence the result.

Corollary 5.4. Let $R$ denote one of the rings $D_{[\rho, 1)}(G, K)$ or $\mathcal{R}(G, K)$. A series $\sum_{\alpha} c_{\alpha} f^{(\alpha)}$ is convergent in $R$ (in either of the topologies) if and only if $\sum_{\alpha} c_{\alpha} \mathbf{b}^{-\alpha}$ defines an element of $R$. Moreover, any element of $R$ can be written in this form. In particular, the weak topology on $R$ is symmetric, in other words it can also be defined by right multiplication by $x_{0}$.

Proof. If $\sum_{\alpha} c_{\alpha} \mathbf{b}^{-\alpha}$ is an element of some $D_{[\rho, 1)}(G, K)$ then so is $\sum_{\alpha} c_{\alpha} f^{(\alpha)}$ as we have

$$
\left\|f^{(\alpha)}\right\|_{r}=\left\|\mathbf{b}^{-\alpha}\right\|_{r}
$$

for any $p^{-1}<r<1$ in $p^{\mathbb{Q}}$. So the series $\sum_{\alpha} c_{\alpha} f^{(\alpha)}$ is convergent in either of the topologies on $R$. On the other hand we have

$$
\left\|f^{(\alpha)}-\mathbf{b}^{-\alpha}\right\|_{r}<\left\|\mathbf{b}^{-\alpha}\right\|_{r}
$$

therefore we can write any element in $D_{[\rho, 1)}(G, K)$ in the required form. This shows that the natural maps

$$
\begin{aligned}
D_{[\rho, 1)}(G, K) & \rightarrow D_{[\rho, 1)}\left(\mathbb{Z}_{p}^{d}, K\right) \\
\mathcal{R}(G, K) & \rightarrow \mathcal{R}\left(\mathbb{Z}_{p}^{d}, K\right)
\end{aligned}
$$

defined by the identification of the two underlying vector spaces on which the rings are defined is a homeomorphism in the weak topology on both sides. The result follows by the similar statement for the other weak topology defined by right multiplication by $x_{0}$.

Now we can state and prove our main theorem in this section.

Theorem 5.5. The rings $D_{[\rho, 1)}(G, K)$ and $\mathcal{R}(G, K)$ are self-dual topological $K$-algebras (in their own weak topology) with respect to the perfect pairing

$$
\begin{aligned}
R \times R & \rightarrow K \\
(x(\mathbf{b}), y(\mathbf{b})) & \mapsto " \text { the constant term of } x(\mathbf{b}) y(\mathbf{b}) . "
\end{aligned}
$$

Here $R$ denotes either of the above rings.

Proof. The above pairing is clearly $K$-bilinear and separately continuous. So it remains to prove that it induces a topological isomorphism

$$
R \rightarrow \operatorname{Hom}_{K}^{c t}(R, K)
$$

with the weak topology on the dual space. Moreover, by the choice of the topologies on both sides, it remains to show that (28) is a bijection. 
First we need to prove that the map (28) is injective, in other words for any $x \neq 0$ in $R$ there exists a $y$ in $R$ such that $x y$ has nonzero constant term. For this fix a $p^{-1}<r<1$ in $p^{\mathbb{Q}}$ such that $x$ is in $D_{[r, 1)}(G, K)$. Write

$$
x(\mathbf{b})=\sum_{\alpha \in \mathbb{Z}^{d}} x_{\alpha} \mathbf{b}^{\alpha}
$$

and let $\beta$ be such that $\|x\|_{r}=\left|x_{\beta}\right| r^{|\beta|}$. We claim that we may choose $y=\mathbf{b}^{-\beta}$. Indeed, we have

$$
\left\|x(\mathbf{b}) y(\mathbf{b})-\sum_{\alpha} x_{\alpha} \mathbf{b}^{\alpha-\beta}\right\|_{r}<\|x(\mathbf{b}) y(\mathbf{b})\|_{r}=\left|x_{\beta}\right| .
$$

So in particular the constant term of $x(\mathbf{b}) y(\mathbf{b})$ differs from $x_{\beta}$ by something with absolute value less than $\left|x_{\beta}\right|$, hence it is nonzero.

For the surjectivity let $\lambda: R \rightarrow K$ be $K$-linear and continuous with respect to the weak topology on $R$ and the natural topology on $K$. Then $U:=\left\{\left.y \in R|| \lambda(y)\right|_{K}<1\right\}$ is open in $R$. Thus, there are $x_{1}, \ldots, x_{s} \in R$, and there is $\varepsilon>0$ such that

$$
\bigcap_{i=1}^{s}\left\{y \in R||^{\prime \prime} \text { constant term of }\left.x_{i} y^{\prime \prime}\right|_{K}<\varepsilon\right\} \subset U \text {. }
$$

Put $\lambda_{x_{i}}(y):=$ "constant term of $x_{i} y$ ". Consider $y \in V:=\bigcap_{i=1}^{s} \operatorname{Ker}\left(\lambda_{x_{i}}\right)$. Then $K y \subset V \subset U$, and $|\lambda(c y)|<1$ for any $c \in K$ implies $\lambda(y)=0$. Therefore $\lambda$ factors through $R / V$. Next, the map $R / V \rightarrow K^{s}, y+V \mapsto\left(\lambda_{x_{1}}(y), \ldots, \lambda_{x_{s}}(y)\right)$, is injective showing that $\lambda$ is a linear combination $a_{1} \lambda_{x_{1}}+\cdots+a_{s} \lambda_{x_{s}}$ of the $\lambda_{x_{i}}$, and thus $\lambda=\lambda_{x}$ with $x=a_{1} x_{1}+\cdots+a_{s} x_{s}$.

\subsection{The nice topology}

The problem with the weak topology is that it is artificially constructed forcing the ring to be self-dual. Moreover, its restriction to $D(G, K)$ - even in the Robba-ring case - does not give back the Fréchet topology on $D(G, K)$, since any open lattice in the weak topology contains a subspace of finite codimension. However, we are going to construct a third topology on the generalized Robba ring $\mathcal{R}(G, K)$, the "nice" topology which has the following properties.

(i) It is stronger than the weak topology, but weaker than the inductive limit topology.

(ii) The $\operatorname{ring} \mathcal{R}(G, K)$ is self-dual in the nice topology with respect to the pairing

$$
\begin{aligned}
\mathcal{R}(G, K) \times \mathcal{R}(G, K) & \rightarrow K \\
(x, y) & \mapsto \text { "the constant term of } x y . "
\end{aligned}
$$

(iii) On the classical Robba $\operatorname{ring} \mathcal{R}\left(\mathbb{Z}_{p}, K\right)$ the nice topology coincides with the inductive limit topology.

(iv) The subspace topology on the distribution algebra $D(G, K)$ equals the usual Fréchet topology. 
The construction is the following. For any fixed element

$$
x_{0}=\sum_{\alpha \in \mathbb{Z}^{d}} c_{0, \alpha} \mathbf{b}^{\alpha}
$$

in $\mathcal{R}(G, K)$ we take the $o_{K}$-submodule

$$
L_{x_{0}}:=\left\{\sum_{\alpha \in \mathbb{Z}^{d}} d_{\alpha} \mathbf{b}^{\alpha} \text { such that }\left|c_{0,-\alpha}\right|\left|d_{\alpha}\right| \leq 1 \text { for any } \alpha \in \mathbb{Z}^{d}\right\}
$$

in $\mathcal{R}(G, K)$.

Lemma 5.6. The set $L_{x_{0}}$ defined in (30) is a lattice in $\mathcal{R}(G, K)$.

Proof. We need to show that for any nonzero element $x=\sum c_{\alpha} \mathbf{b}^{\alpha}$ in $\mathcal{R}(G, K)$ there exists a constant $c$ in the ring of integers $o_{K}$ such that $c x$ lies in $L_{x_{0}}$. We can take the product $x \circ x_{0}$ in the commutative ring $\mathcal{R}\left(\mathbb{Z}_{p}^{d}, K\right)$ which has the same underlying set as $\mathcal{R}(G, K)$. The constant term of $x \circ x_{0}$ is by definition

$$
\sum_{\alpha \in \mathbb{Z}^{d}} c_{\alpha} c_{0,-\alpha},
$$

so this sum is convergent and hence bounded. Therefore there exists a constant $c$ in $o_{K}$ such that the element $c x$ lies in $L_{x_{0}}$. Note that this is a stronger assumption than just that the constant term of $c x \circ x_{0}$ be integral.

Now we define a lattice open in the nice topology if it contains a lattice of the form $L_{x_{0}}$. This gives a locally convex topology on $\mathcal{R}(G, K)$ since for any elements $x_{1}, \ldots, x_{m}$ in $\mathcal{R}(G, K)$ there exists an element $x_{0}$ in $\mathcal{R}(G, K)$ such that we have

$$
L_{x_{1}} \cap \cdots \cap L_{x_{m}}=L_{x_{0}} .
$$

Indeed, we can take $x_{0}$ such that its coefficients satisfy

$$
\left|c_{0, \alpha}\right|=\max _{1 \leq i \leq m}\left|c_{i, \alpha}\right|
$$

where $c_{i, \alpha}$ is the coefficient of $\mathbf{b}^{\alpha}$ in $x_{i}$.

Lemma 5.7. The nice topology on $\mathcal{R}(G, K)$ is stronger than the weak topology, but weaker than the inductive limit topology.

Proof. The lattice $L_{x_{0}}$ is certainly contained in the lattice

$$
\left\{x \in \mathcal{R}(G, K):\left|\left(f_{0}, x\right)\right| \leq 1\right\}
$$

where $f_{0}=\sum_{\alpha} c_{0,-\alpha} f^{(\alpha)}$ with $x_{0}=\sum_{\alpha} c_{0, \alpha} \mathbf{b}^{\alpha}$ which form a base of neighbourhood of the origin in the weak topology.

For the second statement choose real numbers $\rho<\rho_{1}<1$ such that $x_{0}$ is in $D_{[\rho, 1)}(G, K)$. Then by definition (30) $L_{x_{0}} \cap D_{[\rho, 1)}(G, K)$ contains the lattice

$$
\left\{x \in \mathcal{R}(G, K):\|x\|_{\rho, \rho_{1}} \leq \frac{1}{\left\|x_{0}\right\|_{\rho, \rho_{1}}}\right\}
$$

showing that $L_{x_{0}}$ is open in the inductive limit topology of $\mathcal{R}(G, K)$. 
Lemma 5.8. The map

$$
\mathcal{R}(G, K) \rightarrow \operatorname{Hom}_{K}^{c t, n i c e}(\mathcal{R}(G, K), K)
$$

induced by the pairing (29) is surjective.

Proof. First of all one needs to verify that the pairing (29) is separately continuous. Indeed, this pairing is separately continuous even in the weak topology and the nice topology is stronger.

The proof of the surjectivity is similar to the proof of Theorem 5.5, Take a continuous linear functional $\varphi$ from $\mathcal{R}(G, K)$ to $K$. Since $\varphi$ is continuous in the nice topology there exists an element $y$ in $\mathcal{R}(G, K)$ such that $\varphi^{-1}\left(o_{K}\right) \supseteq L_{y}$. Write $y$ in the form

$$
y=\sum_{\alpha \in \mathbb{Z}^{d}} y_{\alpha} \mathbf{b}^{\alpha} .
$$

By definition of $L_{y}$ we have $\left|\varphi\left(\mathbf{b}^{\alpha}\right)\right| \leq y_{-\alpha}$ for all $\alpha \in \mathbb{Z}^{d}$. This means that the series

$$
\sum_{\alpha \in \mathbb{Z}^{d}} \varphi\left(\mathbf{b}^{\alpha}\right) f^{(\alpha)}
$$

defines an element $f_{\varphi}$ in $\mathcal{R}(G, K)$ such that $f_{\varphi}$ maps to the functional $\varphi$ under the map (31).

Lemma 5.9. The subspace topology on $D(G, K)$ in the nice topology coincides with its canonical Fréchet topology.

Proof. Since the restriction of the inductive limit topology of $\mathcal{R}(G, K)$ on $D(G, K)$ equals its canonical topology it suffices to show that whenever $L \subset D(G, K)$ is an open lattice in the Fréchet topology then there exists an element $x(L)$ in $\mathcal{R}(G, K)$ such that $L_{x(L)} \cap D(G, K) \subseteq L$. Now since $L$ is open in the Fréchet topology there exist a real numbers $p^{-1}<\rho<1$ and an integer $l$ such that $y$ is in $L$ whenever $\|y\|_{\rho} \leq p^{-l}$ for the element $y$ in $D(G, K)$. Now we define

$$
\begin{aligned}
x & :=\sum_{\alpha \in \mathbb{N}_{0}^{d}} x_{-\alpha} \mathbf{b}^{-\alpha} \in \mathcal{R}\left(G, \mathbb{Q}_{p}\right) \text { with } \\
p^{l} \rho^{|\alpha|} \leq\left|x_{-\alpha}\right| & <p^{l+1} \rho^{|\alpha|} .
\end{aligned}
$$

Note that (32) determines $x_{-\alpha}$ in $\mathbb{Q}_{p}$ up to a unit in $\mathbb{Z}_{p}^{\times}$-otherwise we choose $x_{-\alpha}$ arbitrarily. Now we see that $x$ is an element in $D_{(\rho, 1)}(G, K)$ and we have $L_{x(L)} \cap D(G, K) \subseteq L$ by construction.

Proposition 5.10. The multiplication of the ring $\mathcal{R}(G, K)$ is separately continuous in the nice topology.

Proof. By the left-right symmetry of the nice topology it suffices to show that for any fixed elements $x_{0}$ and $y_{0}$ in $\mathcal{R}(G, K)$ the pre-image $y_{0}^{-1} L_{x_{0}}$ of the lattice $L_{x_{0}}$ under the left multiplication by $y_{0}$ is open. $y_{0}^{-1} L_{x_{0}}$ is clearly a lattice in $\mathcal{R}(G, K)$. Moreover, the Laurent polynomials are dense in $\mathcal{R}(G, K)$ in the inductive limit topology and $y_{0}^{-1} L_{x_{0}}$ is open (and 
hence closed) in the inductive limit topology by the continuity of the multiplication by $y_{0}$. Therefore it suffices to show that there exists an element

$$
z_{0}=\sum_{\alpha \in \mathbb{Z}^{d}} a_{\alpha} \mathbf{b}^{\alpha}
$$

in $\mathcal{R}(G, K)$ such that for any $\alpha$

$$
\begin{aligned}
\left|a_{\alpha}\right| s_{\alpha} & \geq 1 \quad \text { where } \\
s_{\alpha} & :=\sup \left\{|s|: s \mathbf{b}^{-\alpha} \in y_{0}^{-1} L_{x_{0}}\right\} \in \mathbb{R}^{>0} \cup\{+\infty\} .
\end{aligned}
$$

Indeed, if $z_{0}$ satisfies (33) then $L_{z_{0}} \subseteq y_{0}^{-1} L_{x_{0}}$. This condition is equivalent to that there exists a real number $p^{-1}<\rho<1$ such that the set

$$
\left\{\frac{\rho_{1}^{|\alpha|}}{s_{\alpha}}\right\}_{\alpha \in \mathbb{Z}^{d}}
$$

converges to 0 for any $1>\rho_{1} \geq \rho$ because this is the convergence condition for the coefficients $a_{\alpha}$ so that $z_{0}$ lies in $D_{[\rho, 1)}(G, K)$. We determine the real number $\rho$ the following way. By Lemma $5.7 L_{x_{0}}$ is open in the inductive limit topology of $\mathcal{R}(G, K)$, hence for any fixed $p^{-1}<\rho_{2}<1$ there exists a $\rho_{2}<\rho_{3}<1$ such that $L_{x_{0}} \cap D_{\left[\rho_{2}, 1\right)}(G, K)$ contains the lattice

$$
\left\{x \in D_{\left[\rho_{2}, 1\right)}(G, K):\|x\|_{\rho_{2}, \rho_{3}} \leq c\left(x_{0}, \rho_{2}, \rho_{3}\right)\right\}
$$

where $c\left(x_{0}, \rho_{2}, \rho_{3}\right)$ is a positive real number depending on $x_{0}, \rho_{2}$, and $\rho_{3}$. We pick $\rho_{2}$ such that both $y_{0}$ and $x_{0}$ lie in $D_{\left[\rho_{2}, 1\right)}(G, K)$ and claim that we can take any $\rho$ which is bigger than $\rho_{3}$. To show this fix $\rho_{1}>\rho_{3}$ and choose a real number $\varepsilon>0$. We need to verify that for all but finitely many $\alpha$ in $\mathbb{Z}^{d}$ we have

$$
\frac{\rho_{1}^{|\alpha|}}{s_{\alpha}}<\varepsilon
$$

Equivalently, by definition (34) of $s_{\alpha}$ we need to find a coefficient $c_{-\alpha}$ in $\mathbb{Q}_{p}$ for all but finitely many $\alpha$ in $\mathbb{Z}^{d}$ with the properties

(i) $c_{-\alpha} y_{0} \mathbf{b}^{-\alpha}$ lies in $L_{x_{0}}$, and

(ii) $\left|c_{-\alpha}\right|>\frac{\rho_{1}^{|\alpha|}}{\varepsilon}$.

At first we show that there exists an integer $n_{0}$ such that whenever $|\alpha|<n_{0}$ then there is a $c_{-\alpha}$ with properties $(i)$ and $(i i)$. Indeed, we choose $n_{0}<0$ small enough so that

$$
\left(\frac{\rho_{1}}{\rho_{3}}\right)^{n_{0}} \leq \frac{\varepsilon c\left(x_{0}, \rho_{2}, \rho_{3}\right)}{p\left\|y_{0}\right\|_{\rho_{2}, \rho_{3}}}
$$

Since $\rho_{1}>\rho_{3}$ there exists such an $n_{0}$. Moreover, whenever $|\alpha|<n_{0}$ then we have

$$
p \frac{\rho_{1}^{|\alpha|}}{\varepsilon}\left\|y_{0} \mathbf{b}^{-\alpha}\right\|_{\rho_{2}, \rho_{3}} \leq p \frac{\rho_{1}^{|\alpha|}}{\varepsilon}\left\|y_{0}\right\|_{\rho_{2}, \rho_{3}}\left\|\mathbf{b}^{-\alpha}\right\|_{\rho_{2}, \rho_{3}}=p \frac{\rho_{1}^{|\alpha|}}{\varepsilon}\left\|y_{0}\right\|_{\rho_{2}, \rho_{3}} \rho_{3}^{-|\alpha|} \leq c\left(x_{0}, \rho_{2}, \rho_{3}\right),
$$


whence we may take any $c_{-\alpha}$ such that

$$
\frac{\rho_{1}^{|\alpha|}}{\varepsilon}<\left|c_{-\alpha}\right| \leq p \frac{\rho_{1}^{|\alpha|}}{\varepsilon}
$$

and $c_{-\alpha} y_{0} \mathbf{b}^{-\alpha}$ will lie in $L_{x_{0}}$ by (35). Indeed, there exists a $c_{-\alpha}$ in $\mathbb{Q}_{p}$ with the required absolute value in (37).

On the other hand we now prove that there also exists an integer $n_{1}>0$ such that there is a $c_{-\alpha}$ with properties $(i)$ and $(i i)$ whenever $|\alpha|>n_{1}$. For this we take a real number $1>\rho_{4}>\rho_{1}$. The lattice $L_{x_{0}} \cap D_{\left[\rho_{4}, 1\right)}(G, K)$ is open in the Fréchet topology of $D_{\left[\rho_{4}, 1\right)}(G, K)$ by Lemma 5.7. This means that there exist real numbers $1>\rho_{5}>\rho_{4}$ and $c\left(x_{0}, \rho_{4}, \rho_{5}\right)$ such that the lattice

$$
\left\{x \in D_{\left[\rho_{4}, 1\right)}(G, K):\|x\|_{\rho_{4}, \rho_{5}} \leq c\left(x_{0}, \rho_{4}, \rho_{5}\right)\right\}
$$

is contained in $L_{x_{0}} \cap D_{\left[\rho_{4}, 1\right)}(G, K)$. We choose $n_{1}$-in a similar way as above in (36) - such that

$$
\left(\frac{\rho_{1}}{\rho_{4}}\right)^{n_{0}} \leq \frac{\varepsilon c\left(x_{0}, \rho_{4}, \rho_{5}\right)}{p\left\|y_{0}\right\|_{\rho_{4}, \rho_{5}}} .
$$

Then by the same argument as above there exists a required $c_{-\alpha}$ for any $\alpha$ with degree bigger than $n_{1}$.

Now take an integer $n_{2}$ with $n_{0} \leq n_{2} \leq n_{1}$. It suffices to show that there exists a $c_{-\alpha}$ satisfying $(i)$ and $(i i)$ for all but finitely many $\alpha$ with fixed degree $|\alpha|=n_{2}$. At first we write

$$
x_{0}=\sum_{\gamma \in \mathbb{Z}^{d}} c_{0, \gamma} \mathbf{b}^{\gamma}
$$

and put

$$
A\left(x_{0}, y_{0}, n_{2}\right):=\left\{\alpha \in \mathbb{Z}^{d}:|\alpha|=n_{2} \text { and there does not exist a } c_{-\alpha} \text { satisfying }(i) \text { and }(i i)\right\} .
$$

By construction of $L_{x_{0}}$ we obtain that

$$
\begin{aligned}
L_{x_{0}} & =\bigcap_{\gamma \in \mathbb{Z}^{d}} L_{c_{0, \gamma} \mathbf{b}^{\gamma}} \quad \text { and } \\
A\left(x_{0}, y_{0}, n_{2}\right) & =\bigcup_{\gamma \in \mathbb{Z}^{d}} A\left(c_{0, \gamma} \mathbf{b}^{\gamma}, y_{0}, n_{2}\right) .
\end{aligned}
$$

Now we show that for all but finitely many $\gamma$ the set $A\left(c_{0, \gamma} \mathbf{b}^{\gamma}, y_{0}, n_{2}\right)$ is empty. Indeed, by Lemma 5.7 $A\left(c_{0, \gamma} \mathbf{b}^{\gamma}, y_{0}, n_{2}\right)$ is empty whenever we have

$$
p \frac{\rho_{1}^{n_{2}}}{\varepsilon}\left\|y_{0}\right\|_{\rho_{2}, \rho_{3}} \max \left(\rho_{2}^{-n_{2}}, \rho_{3}^{-n_{2}}\right) \leq \frac{1}{\left\|c_{0, \gamma} \mathbf{b}^{\gamma}\right\|_{\rho_{2}, \rho_{3}}}
$$

because $L_{c_{0, \gamma} \mathbf{b} \gamma}$ contains the lattice

$$
\left\{x \in D_{\left[\rho_{2}, 1\right)}(G, K):\|x\|_{\rho_{2}, \rho_{3}} \leq \frac{1}{\left\|c_{0, \gamma} \mathbf{b}^{\gamma}\right\|_{\rho_{2}, \rho_{3}}}\right\} .
$$


On the other hand $x_{0}$ is in $D_{\left[\rho_{2}, 1\right)}(G, K)$ by the choice of $\rho_{2}$, so (39) is satisfied for all but finitely many $\gamma$ in $\mathbb{Z}^{d}$. Therefore we may assume without loss of generality that $x_{0}$ is of the form $x_{0}=c_{0, \gamma_{0}} \mathbf{b}^{\gamma_{0}}$ for some $\gamma_{0}$ in $\mathbb{Z}^{d}$ and $c_{0, \gamma_{0}}$ in $K$ since (38) is essentially a finite union. Moreover, we have

$$
\left(f^{\left(-\gamma_{0}\right)} y_{0}\right)^{-1} L_{c_{0, \gamma_{0}}}=y_{0}^{-1} L_{c_{0, \gamma_{0}} \mathbf{b}^{\gamma_{0}}}
$$

so we may, as well, assume that $\gamma_{0}=(0, \ldots, 0)$. Now we write

$$
y_{0}=\sum_{\beta \in \mathbb{Z}^{d}} y_{0, \beta} \mathbf{b}^{\beta}
$$

By a similar argument as above for $y_{0}$ instead of $x_{0}$ we get

$$
A\left(c_{0, \gamma_{0}}, y_{0}, n_{2}\right)=\bigcup_{\beta \in \mathbb{Z}^{d}} A\left(c_{0, \gamma_{0}}, y_{0, \beta} \mathbf{b}^{\beta}, n_{2}\right)
$$

and for all but finitely many $\beta$ in $\mathbb{Z}^{d}$ the set $A\left(c_{0, \gamma_{0}}, y_{0, \beta} \mathbf{b}^{\beta}, n_{2}\right)$ is empty. Hence we may assume that $y_{0}=y_{0, \beta_{0}} \mathbf{b}^{\beta_{0}}$ for some $\beta_{0}$ in $\mathbb{Z}^{d}$ and the result follows from Lemma 5.1 (ii).

So putting everything together we get the following

Corollary 5.11. The generalized Robba ring $\mathcal{R}(G, K)$ is a self-dual topological $K$-algebra in the nice topology.

Proposition 5.12. The nice topology on the classical Robba ring $\mathcal{R}\left(\mathbb{Z}_{p}, K\right)$ coincides with the inductive limit topology.

Proof. One direction follows from Lemma 5.7. For the other direction take a lattice $L$ in $\mathcal{R}\left(\mathbb{Z}_{p}, K\right)$ which is open in the inductive limit topology. We need to show that there exists an element $x_{0}$ in $\mathcal{R}\left(\mathbb{Z}_{p}, K\right)$ such that $L_{x_{0}} \subseteq L$. Since $L$ is open in the inductive limit topology, for any $p^{-1}<\rho_{1}<1$ there exists a $\rho_{2}>\rho_{1}$ such that $L \cap D_{\left[\rho_{1}, 1\right)}\left(\mathbb{Z}_{p}, K\right)$ contains the lattice

$$
\left\{x \in D_{\left[\rho_{1}, 1\right)}\left(\mathbb{Z}_{p}, K\right):\|x\|_{\rho_{1}, \rho_{2}} \leq c_{L, \rho_{1}}\right\}
$$

for some positive real number $c_{L, \rho_{1}}$. Put

$$
s_{n}:=\sup \left(|s|: s T^{n} \text { is in } L\right) \in \mathbb{R}^{>0} \cup\{+\infty\} .
$$

We need to verify that there exists a real number $p^{-1}<\rho_{0}<1$ such that

$$
\begin{aligned}
& \lim _{n \rightarrow \infty} \frac{\rho^{n}}{s_{-n}}=0 \quad \text { and } \\
& \lim _{n \rightarrow-\infty} \frac{\rho^{n}}{s_{-n}}=0
\end{aligned}
$$

for all $1>\rho>\rho_{0}$. We show that $\rho_{0}:=\rho_{2}$ will do. Take a real number $\rho$ such that $1>\rho>\rho_{0}$. Choose $1>\rho_{3}>\rho$ and $1>\rho_{4}>\rho_{3}$ such that $L \cap D_{\left[\rho_{3}, 1\right)}\left(\mathbb{Z}_{p}, K\right)$ contains the lattice

$$
\left\{x \in D_{\left[\rho_{3}, 1\right)}\left(\mathbb{Z}_{p}, K\right):\|x\|_{\rho_{3}, \rho_{4}} \leq c_{L, \rho_{3}}\right\} .
$$


So we obtain that

$$
\begin{aligned}
& s_{-n} \geq \frac{c_{L, \rho_{1}}}{p \max \left(\rho_{1}^{-n}, \rho_{2}^{-n}\right)} \quad \text { and } \\
& s_{-n} \geq \frac{c_{L, \rho_{3}}}{p \max \left(\rho_{3}^{-n}, \rho_{4}^{-n}\right)} .
\end{aligned}
$$

Indeed, there exist coefficients $c_{1,-n}$ and $c_{2,-n}$ in $\mathbb{Q}_{p}$ such that

$$
\begin{aligned}
& \frac{c_{L, \rho_{1}}}{\max \left(\rho_{1}^{-n}, \rho_{2}^{-n}\right)}>\quad c_{1,-n} \geq \frac{c_{L, \rho_{1}}}{p \max \left(\rho_{1}^{-n}, \rho_{2}^{-n}\right)} \quad \text { and } \\
& \frac{c_{L, \rho_{3}}}{\max \left(\rho_{3}^{-n}, \rho_{4}^{-n}\right)}>\quad c_{2,-n} \geq \frac{c_{L, \rho_{3}}}{p \max \left(\rho_{3}^{-n}, \rho_{4}^{-n}\right)}
\end{aligned}
$$

whence both $c_{i,-n} T^{-n}$ are in $L$ for $i=1,2$ by (40) and (43), resp. Now (41) follows from (45), and (42) from (44).

Corollary 5.13. Whenever $\operatorname{dim} G>1$ the dual space of $\mathcal{R}(G, K)$ in the inductive limit topology is strictly bigger than the dual in the nice topology. In particular these topologies do not coincide.

Proof. We show that the linear functional

$$
\begin{aligned}
\varphi: \mathcal{R}(G, K) & \rightarrow K \\
\sum_{\alpha \in \mathbb{Z}^{d}} d_{\alpha} \mathbf{b}^{\alpha} & \mapsto \sum_{\alpha \in \mathbb{Z}^{d},|\alpha|=0} d_{\alpha}
\end{aligned}
$$

is continuous in the inductive limit topology, but not in the nice topology. The latter statement is clear as this functional would correspond to an element in $\mathcal{R}(G, K)$ by Lemma 5.8. However, the nice topology is independent of the uniform pro- $p$ group structure on $G$ and whenever $G$ is commutative this element would have to be of the form

$$
\sum_{|\alpha|=0} \mathbf{b}^{\alpha}
$$

which is not an element of $\mathcal{R}(G, K)$.

For the other statement it suffices to show that the above functional is continuous on each $D_{[\rho, 1)}(G, K)$. This is clear as it satisfies

$$
|\varphi(x)| \leq\|x\|_{r}
$$

for any $\rho \leq r<1$.

\section{Further results on coadmissible modules}

In this section we define an exact functor $R$ from the category of coadmissible (left) modules over the distribution algebra $D(G, K)$ to the category of (left) modules over the generalized Robba ring $\mathcal{R}(G, K)$. We call the image of this functor the category of coadmissible modules over $\mathcal{R}(G, K)$. We show that whenever $N$ is a finitely generated coadmissible 
module over $D(G, K)$ then $\operatorname{Hom}_{\mathcal{R}(G, K)}(R(N), \mathcal{R}(G, K))$ is also coadmissible (ie. lies in the image of the functor $R$ ). By Corollary $5.11 \mathcal{R}(G, K)$ is a self-dual topological $K$-algebra, so we would like to interpret the module $\operatorname{Hom}_{\mathcal{R}(G, K)}(R(N), \mathcal{R}(G, K))$ as the naïve dual space $\operatorname{Hom}_{K}^{c t}(R(N), K)$ with respect to some topology on $R(N)$. For this we equip $\mathcal{R}(G, K)$ with the nice topology. Further, if $M$ is an (abstract) $\mathcal{R}(G, K)$-module then we define the canonical topology on it by taking the strongest locally convex topology on $M$ such that for any fixed element $m_{0}$ in $M$ the map

$$
\begin{aligned}
\mathcal{R}(G, K) & \rightarrow M \\
x & \mapsto x m_{0}
\end{aligned}
$$

is continuous in the nice topology of $\mathcal{R}(G, K)$.

Lemma 6.1. The action of $\mathcal{R}(G, K)$ on $M$ is separately continuous in the canonical topology of $M$. Moreover, any algebraic homomorphism between two modules $M$ and $N$ is continuous in the canonical topology. In particular the canonical topology on the module $\mathcal{R}(G, K)$ over itself coincides with the nice topology.

Proof. For the first statement we need to verify that for any fixed $x_{0}$ in $\mathcal{R}(G, K)$ the map

$$
\begin{aligned}
M & \rightarrow M \\
m & \mapsto x_{0} m
\end{aligned}
$$

is continuous. By the construction of the topology this follows from the continuity of the map

$$
\begin{aligned}
\mathcal{R}(G, K) & \rightarrow M \\
x & \mapsto x x_{0} m_{0}
\end{aligned}
$$

for any fixed $x_{0}$ in $\mathcal{R}(G, K)$ and $m_{0}$ in $M$. The second statement follows similarly.

So from now on any $\mathcal{R}(G, K)$-module will be equipped with its canonical topology and we understand continuous maps as "continuous in the canonical topology".

Lemma 6.2. Let $M$ be any module over $\mathcal{R}(G, K)$ with its canonical topology. Then the map

$$
\mu: \operatorname{Hom}_{\mathcal{R}(G, K)}(M, \mathcal{R}(G, K)) \rightarrow \operatorname{Hom}_{K}^{c t}(M, K)
$$

given by $(\mu(\varphi))(m):=$ "the constant term of $\varphi(m)$ " is a K-linear bijection.

Proof. By Lemma 6.1 the functional $\mu(\varphi)$ is indeed continuous showing that the map $\mu$ is well-defined. The injectivity of $\mu$ follows from the non-degeneracy of the pairing on $\mathcal{R}(G, K)$. On the other hand, if $\bar{\varphi}$ is a continuous linear functional on $M$ then the map

$$
\begin{aligned}
\varphi_{m_{0}}: \mathcal{R}(G, K) & \rightarrow K \\
\varphi_{m_{0}}(r) & :=\bar{\varphi}\left(r m_{0}\right)
\end{aligned}
$$

is continuous in the nice topology on $\mathcal{R}(G, K)$ and therefore has to come from the right multiplication by an element $r_{0}=: \varphi\left(m_{0}\right)$ (by Corollary 5.11) and the Lemma follows. 
Our next observation is the following

Proposition 6.3. Let $P$ be a projective coadmissible left $D(G, K)$-module. Then we have

$$
\operatorname{Hom}_{D(G, K)}(P, D(G, K)) \otimes_{D(G, K)} \mathcal{R}(G, K) \cong \operatorname{Hom}_{K}^{c t}\left(\mathcal{R}(G, K) \otimes_{D(G, K)} P, K\right)
$$

Proof. We choose another projective $D(G, K)$-module $Q$ such that $P \oplus Q \cong D(G, K)^{m}$ for some integer $m$. Note that $P$ is finitely generated by Proposition 3.10, Now we have the following isomorphisms

$$
\begin{array}{r}
\operatorname{Hom}(P, D(G, K)) \otimes \mathcal{R}(G, K) \oplus \operatorname{Hom}(Q, D(G, K)) \otimes \mathcal{R}(G, K) \stackrel{\sim}{\longrightarrow} \\
\stackrel{\sim}{\longrightarrow}(\operatorname{Hom}(P, D(G, K)) \oplus \operatorname{Hom}(Q, D(G, K)) \otimes \mathcal{R}(G, K) \stackrel{\sim}{\longrightarrow} \\
\stackrel{\sim}{\longrightarrow} \operatorname{Hom}\left(D(G, K)^{m}, D(G, K)\right) \otimes \mathcal{R}(G, K) \stackrel{\sim}{\longrightarrow} \operatorname{Hom}\left(\mathcal{R}(G, K)^{m}, \mathcal{R}(G, K)\right) \stackrel{\sim}{\longrightarrow} \\
\stackrel{\sim}{\longrightarrow} \operatorname{Hom}(\mathcal{R}(G, K) \otimes P \oplus \mathcal{R}(G, K) \otimes Q, \mathcal{R}(G, K)) \stackrel{\sim}{\longrightarrow} \\
\stackrel{\sim}{\longrightarrow} \operatorname{Hom}(\mathcal{R}(G, K) \otimes P, \mathcal{R}(G, K)) \oplus \operatorname{Hom}(\mathcal{R}(G, K) \otimes Q, \mathcal{R}(G, K)) .
\end{array}
$$

Moreover, the isomorphism from the first term to the last term is the direct sum of the maps

$$
\begin{array}{ll}
\operatorname{Hom}(P, D(G, K)) \otimes \mathcal{R}(G, K) & \rightarrow \operatorname{Hom}(P \otimes \mathcal{R}(G, K), \mathcal{R}(G, K)) \\
\operatorname{Hom}(Q, D(G, K)) \otimes \mathcal{R}(G, K) & \rightarrow \operatorname{Hom}(Q \otimes \mathcal{R}(G, K), \mathcal{R}(G, K))
\end{array} \quad \text { and }
$$

so both these maps are isomorphisms and the result follows by Lemmata 6.1 and 6.2 .

In the rest of this section we are going to generalize the above observation to any finitely generated coadmissible module over the distribution algebra $D(G, K)$.

For that we need the following

Lemma 6.4. Let $G$ be a uniform pro-p group. Then for any $p^{-1}<\rho_{1}<\rho_{2}<1$ in $p^{\mathbb{Q}}$ the inclusion

$$
D_{\rho_{2}}(G, K) \hookrightarrow D_{\left[\rho_{1}, \rho_{2}\right]}(G, K)
$$

of rings is flat.

Proof. The proof is similar to that of Theorem 4.3. We choose a $\rho_{0}$ in the intersection of $p^{\mathbb{Q}}$ with the open interval $\left(p^{-1}, \rho_{1}\right)$ and show at first that $D_{\left(\rho_{0}, \rho_{2}\right]^{b d}}(G, K)$ is flat over $D_{\rho_{2}}(G, K)$. We take the filtration $F_{\rho_{2}}^{s}$ induced by the norm $\|\cdot\|_{\rho_{2}}$ on $D_{\rho_{2}}(G, K)$ and $F_{\rho_{0}, \rho_{2}}^{0} D_{\left(\rho_{0}, \rho_{2}\right]^{b d}}(G, K)$. Both are complete with respect to this filtration. Moreover, $F_{\rho_{2}}^{0} D_{\rho_{2}}(G, K)$ is contained in $F_{\rho_{0}, \rho_{2}}^{0} D_{\left(\rho_{0}, \rho_{2}\right]^{b d}}(G, K)$ and we have

$$
\mathbb{Q}_{p} \otimes_{\mathbb{Z}_{p}} F_{\rho_{2}}^{0} D_{\rho_{2}}(G, K)=D_{\rho_{2}}(G, K) \text { and } \mathbb{Q}_{p} \otimes_{\mathbb{Z}_{p}} F_{\rho_{0}, \rho_{2}}^{0} D_{\left(\rho_{0}, \rho_{2}\right]^{b d}}(G, K)=D_{\left(\rho_{0}, \rho_{2}\right]^{b d}}(G, K) .
$$

So by Proposition 1.2 in [ST5] it suffices to show that the associated graded map

$$
\operatorname{gr}_{\rho_{2}}^{\cdot} F_{\rho_{2}}^{0} D_{\rho_{2}}(G, K) \hookrightarrow \operatorname{gr}_{\rho_{2}}^{\cdot} F_{\rho_{0}, \rho_{2}}^{0} D_{\left(\rho_{0}, \rho_{2}\right]^{b d}}(G, K)
$$

is flat. Since the norm $\|\cdot\|_{\rho_{2}}$ is multiplicative both these rings are integral domains. Moreover, we may assume without loss of generality that $\rho_{2}$ is an integral power of the absolute value of the uniformizer in $K$ as we may replace $K$ by a finite extension. So the graded ring 
$\operatorname{gr}_{\rho_{2}}^{\cdot} F_{\rho_{2}}^{0} D_{\rho_{2}}(G, K)$ is isomorphic to the polynomial ring $k\left[X_{0}, X_{1}, \ldots, X_{d}\right]$ and $\operatorname{gr}_{\rho_{2}} F_{\rho_{0}, \rho_{2}}^{0} D_{\left(\rho_{0}, \rho_{2}\right]{ }^{b d}}(G, K)$ is its localization at $X_{1}, \ldots, X_{d}$ and therefore flat.

The proof of the flatness of the map

$$
D_{\left(\rho_{0}, \rho_{2}\right]^{b d}}(G, K) \hookrightarrow D_{\left[\rho_{1}, \rho_{2}\right]}(G, K)
$$

is entirely analogous to the proof of Theorem 4.3 and so we omit the details.

Now we fix a sequence $p^{-1}<\rho_{1}<\cdots<\rho_{n}<\cdots<1$ with $\rho_{n} \rightarrow 1$ in $p^{\mathbb{Q}}$ and introduce (for any positive integer $l$ ) the functor $R_{\rho_{l}}$ from the category $\operatorname{Coh}\left(D(G, K), \rho_{n}\right)$ of coherent sheafs for the projective system of noetherian Banach algebras $\left(D_{\rho_{n}}(G, K)\right)_{n \geq 1}$ to the category $\operatorname{Coh}\left(D_{\left[\rho_{l}, 1\right)}(G, K), \rho_{n}\right)$ of coherent sheafs for the system $\left(D_{\left[\rho_{l}, \rho_{n}\right]}(G, K)\right)_{n>l}$ by putting

$$
R_{\rho_{l}}\left(\left(M_{n}\right)_{n \geq 1}\right):=\left(D_{\left[\rho_{l}, \rho_{n}\right]}(G, K) \otimes_{D_{\rho_{n}}(G, K)} M_{n}\right)_{n>l} .
$$

This is an exact functor by Lemma 6.4. Moreover, by the equivalence of the categories of coherent sheafs and coadmissible modules over Fréchet-Stein algebras it can be viewed as a functor from the category $\mathcal{C}_{D(G, K)}$ of coadmissible modules over $D(G, K)$ to the category $\mathcal{C}_{D_{\left[\rho_{l}, 1\right)}(G, K)}$ of coadmissible modules over $D_{\left[\rho_{l}, 1\right)}(G, K)$ since the latter is also a Fréchet-Stein algebra by Theorem 4.3. We denote this functor by the same letter. Moreover, let $R$ be the the injective limit of these functors mapping coadmissible modules $M$ over the distribution algebra $D(G, K)$ to the module

$$
R(M):=\underset{l \rightarrow \infty}{\lim _{l \rightarrow \infty}} R_{\rho_{l}}(M)
$$

over the Robba ring $\mathcal{R}(G, K)$. The functor $R$ is also exact as $\lim _{\longrightarrow}$ is exact. We call a module over the ring $\mathcal{R}(G, K)$ coadmissible if it is in the image of the functor $R$.

Lemma 6.5. Let $M$ be a finitely generated coadmissible module over $D(G, K)$. Then the natural map

$$
\varphi_{l}: D_{\left[\rho_{l}, 1\right)}(G, K) \otimes_{D(G, K)} M \rightarrow R_{l}(M)
$$

is surjective with $D_{\left[\rho_{l}, 1\right)}(G, K)$-torsion kernel for any $l$. In particular if the $D_{\left[\rho_{l}, 1\right)}(G, K)$ module $N$ has no torsion then we have the isomorphism

$$
\operatorname{Hom}_{D_{\left[\rho_{l}, 1\right)}(G, K)}\left(D_{\left[\rho_{l}, 1\right)}(G, K) \otimes_{D(G, K)} M, N\right) \cong \operatorname{Hom}_{D_{\left[\rho_{l}, 1\right)}(G, K)}\left(R_{l}(M), N\right) .
$$

Proof. The image of $\varphi_{l}$ is a coadmissible $D_{\left[\rho_{l}, 1\right)}(G, K)$-module as it is a finitely generated submodule of a coadmissible module. Moreover, for any $n>l$ we have

$$
D_{\left[\rho_{l}, \rho_{n}\right]}(G, K) \otimes \operatorname{Im}(\varphi)=D_{\left[\rho_{l}, \rho_{n}\right]}(G, K) \otimes R_{l}(N) .
$$

Therefore the surjectivity. Let us assume now indirectly that there is an element $x$ in the kernel of $\varphi_{l}$ which is not torsion. This means we have an injective homomorphism from $D_{\left[\rho_{l}, 1\right)}(G, K)$ to $D_{\left[\rho_{l}, 1\right)}(G, K) \otimes_{D(G, K)} M$ mapping 1 to $x$. This contradicts to the flatness of $D_{\left[\rho_{l}, \rho_{n}\right]}(G, K)$ over $D_{\left[\rho_{l}, 1\right)}(G, K)$ which is a consequence of the Fréchet-Stein property. The last statement follows immediately from the long exact sequence of the functor $\operatorname{Hom}_{D_{\left[\rho_{l}, 1\right)}(G, K)}(\cdot, N)$.

Our main result in this section is the following 
Theorem 6.6. Let $M$ be a finitely generated coadmissible module over the distribution algebra $D(G, K)$ of a uniform pro-p group $G$. Then $\operatorname{Hom}_{K}^{c t}(R(M), K)$ is a coadmissible module over $\mathcal{R}(G, K)$.

Proof. We are going to prove that in fact we have

$$
\operatorname{Hom}_{K}^{c t}(R(M), K)=R\left(\operatorname{Hom}_{D(G, K)}(M, D(G, K))\right) .
$$

At first, we clearly have $\operatorname{Hom}_{K}^{c t}(R(M), K)=\operatorname{Hom}_{\mathcal{R}(G, K)}(R(M), \mathcal{R}(G, K))$. Moreover, by definition we also have

$$
\operatorname{Hom}_{\mathcal{R}(G, K)}(R(M), \mathcal{R}(G, K))=\underset{l \rightarrow \infty}{\lim _{l \rightarrow \infty}} \operatorname{Hom}_{D_{\left[\rho_{l}, 1\right)}(G, K)}\left(R_{l}(M), \mathcal{R}(G, K)\right) .
$$

Further, by Lemma 6.5 we obtain

$$
\begin{array}{r}
\operatorname{Hom}_{D_{\left[\rho_{l}, 1\right)}(G, K)}\left(R_{l}(M), \mathcal{R}(G, K)\right)=\operatorname{Hom}_{D_{\left[\rho_{l}, 1\right)}(G, K)}\left(D_{\left[\rho_{l}, 1\right)}(G, K) \otimes_{D(G, K)} M, \mathcal{R}(G, K)\right)= \\
=\operatorname{Hom}_{D(G, K)}(M, \mathcal{R}(G, K)) .
\end{array}
$$

So putting these all together we get

$$
\operatorname{Hom}_{K}^{c t}(R(M), K)=\operatorname{Hom}_{D(G, K)}(M, \mathcal{R}(G, K)) .
$$

On the other hand since $M$ is finitely generated we also have

$$
\operatorname{Hom}_{D(G, K)}(M, \mathcal{R}(G, K))=\underset{l \rightarrow \infty}{\lim _{l \rightarrow \infty}} \operatorname{Hom}_{D(G, K)}\left(M, D_{\left[\rho_{l}, 1\right)}(G, K)\right) .
$$

By Lemma 6.5 once again we also have

$$
\begin{array}{r}
\operatorname{Hom}_{D(G, K)}\left(M, D_{\left[\rho_{l}, 1\right)}(G, K)\right)=\operatorname{Hom}_{D_{\left[\rho_{l}, 1\right)}(G, K)}\left(D_{\left[\rho_{l}, 1\right)}(G, K) \otimes M, D_{\left[\rho_{l}, 1\right)}(G, K)\right)= \\
=\operatorname{Hom}_{D_{\left[\rho_{l}, 1\right)}(G, K)}\left(R_{l}(M), D_{\left[\rho_{l}, 1\right)}(G, K)\right)= \\
=\underbrace{}_{n} \lim _{D_{\left[\rho_{l}, \rho_{n}\right]}(G, K)}\left(D_{\left[\rho_{l}, \rho_{l}\right]}(G, K) \otimes M, D_{\left[\rho_{l}, \rho_{n}\right]}(G, K)\right) .
\end{array}
$$

The last equality follows from the fact that $R_{l}(M)$ is a coadmissible module over the FréchetStein algebra $D_{\left[\rho_{l}, 1\right)}(G, K)$. Now by the flatness of $D_{\left[\rho_{l}, \rho_{n}\right]}(G, K)$ over $D_{\rho_{n}}(G, K)$ and the spectral sequences associated to $\operatorname{Hom}_{D_{\left[\rho_{l}, \rho_{n}\right]}(G, K)}\left(D_{\left[\rho_{l}, \rho_{n}\right]}(G, K) \otimes \cdot, D_{\left[\rho_{l}, \rho_{n}\right]}(G, K)\right)$ and $D_{\left[\rho_{l}, \rho_{n}\right]}(G, K) \otimes$ $\operatorname{Hom}_{D_{\rho_{n}}(G, K)}\left(\cdot, D_{\rho_{n}}(G, K)\right)$ we obtain

$$
\begin{array}{r}
\operatorname{Hom}_{D_{\left[\rho_{l}, \rho_{n}\right]}(G, K)}\left(D_{\left[\rho_{l}, \rho_{n}\right]}(G, K) \otimes M, D_{\left[\rho_{l}, \rho_{n}\right]}(G, K)\right)= \\
=D_{\left[\rho_{l}, \rho_{n}\right]}(G, K) \otimes \operatorname{Hom}_{D_{\rho_{n}}(G, K)}\left(M_{n}, D_{\rho_{n}}(G, K)\right)
\end{array}
$$

and hence

$$
\operatorname{Hom}_{D_{\left[\rho_{l}, 1\right)}(G, K)}\left(R_{l}(M), D_{\left[\rho_{l}, 1\right)}(G, K)\right)=R_{l}\left(\operatorname{Hom}_{D(G, K)}(M, D(G, K))\right) .
$$

The result follows by taking the injective limit. 
Remark. Whenever $G=\mathbb{Z}_{p}$ then any torsion-free coadmissible module over the distribution algebra $D\left(\mathbb{Z}_{p}, K\right)$ is finitely generated and even free, so the above Theorem applies to any coadmissible module over $D\left(\mathbb{Z}_{p}, K\right)$-we may drop the assumption that it is finitely generated. Indeed, any homomorphism in $\operatorname{Hom}_{D\left(\mathbb{Z}_{p}, K\right)}\left(M, D\left(\mathbb{Z}_{p}, K\right)\right)$ factors through the quotient of $M$ by its torsion part. This, however, leaves the following questions open.

Question 1. Does there exist a uniform pro-p group $G$ and a torsion-free coadmissible module over its distribution algebra $D(G, K)$ which is not finitely generated?

Question 2. Does $R_{l}(M)=D_{\left[\rho_{l}, 1\right)}(G, K) \otimes_{D(G, K)} M$ always hold or at least for torsion-free coadmissible modules $M$ ? It is certainly true for projective coadmissible modules.

Question 3. Is there a torsion-free coadmissible module over $D(G, K)$ such that

$$
D_{\left[\rho_{l}, 1\right)}(G, K) \otimes_{D(G, K)} M
$$

is not torsion-free over $D_{\left[\rho_{l}, 1\right)}(G, K)$ ? 


\section{A Appendix by Peter Schneider: Robba rings for com- pact $p$-adic Lie groups}

The Robba ring is a fundamental tool in $p$-adic differential equations and in $p$-adic Galois representations. It is defined as a ring of certain infinite Laurent series in one variable over a $p$-adic field $K$. Conceptually it is related to the cyclotomic $\mathbb{Z}_{p}$-extension of $K$ whose Galois group is isomorphic to the additive group of $p$-adic integers $G=\mathbb{Z}_{p}$. In fact, the Robba ring can be understood in terms of the completed group ring $\mathbb{Z}_{p}[[G]]$ by a process of localization and completion.

Recent developments in the theory of $p$-adic Galois representations require the use of more general compact $p$-adic Lie groups $G$. In particular $G$ might be nonabelian like $G=G L_{n}\left(\mathbb{Z}_{p}\right)$. It then becomes a natural question whether an analog of the Robba ring exists in this situation, possibly being constructed out of the completed group ring $\mathbb{Z}_{p}[[G]]$. But $\mathbb{Z}_{p}[[G]]$, in general, will be noncommutative. Hence localization becomes too difficult a process. The idea of these notes grew out of the technique of algebraic microlocalization.

In the first section we will adapt microlocalization to the framwork of $p$-adic Banach algebras. This means that ring filtrations are replaced by multiplicative nonarchimedean norms. More importantly, for the application we have in mind, it is crucial to generalize the theory in such a way that the microlocalization can be preformed simultaneously with respect to finitely many such norms. Apparently this has not been done in the algebraic context of ring filtrations.

In the second section we apply this new technique to construct, under mild assumptions on $G$, various rings out of $\mathbb{Z}_{p}[[G]]$ culminating in a ring $R(G, K)$ which we call a Robba ring of $G$. Actually the ring $R(G, K)$ does depend, which we suppress in the notation, on the choice of a global coordinate system for the $p$-adic Lie group $G$. That such a phenomenon occurs in higher dimensions might not be too surprising.

In the third section we show that various variants of the Robba ring, which classically play an important role, also exist in our general setting.

The constructions in these notes were first presented at a workshop at Münster in 2005. Due to the lack of applications they were not published at the time. Given the progress made by G. Zábrádi on structural properties of these rings it seemed appropriate to add these essentially unchanged notes as an appendix to his paper.

\section{A.1 Generalized microlocalization of quasi-abelian normed algebras}

Let $K$ be a nonarchimedean complete field with absolute value || . To motivate the construction in this section we consider the Tate algebra $K\langle T\rangle$ over $K$, i.e., the ring of power series $f(T)=\sum_{n \geq 0} \lambda_{n} T^{n}$ over $K$ in one variable $T$ which converge on the closed unit disk. Its natural norm is the Gauss norm given by

$$
|f|_{1}:=\sup _{n \geq 0}\left|\lambda_{n}\right|
$$

But for any $0<r \leq 1$ we also have the norm

$$
|f|_{r}:=\sup _{n \geq 0}\left|\lambda_{n}\right|
$$


on $K\langle T\rangle$. If some power of $r$ lies in the value group $\left|K^{\times}\right|$then the completion of $K\langle T\rangle$ with respect to the norm ||$_{r}$ is the algebra of analytic functions on the closed disk of radius $r$ around the origin. On the other hand, if we first invert the variable $T$ and then complete with respect to the norm ||$_{1}$ then we obtain the analytic functions on the unit circle. Microlocalization can be viewed as a generalization of this latter construction to certain noncommutative normed algebras. Finally, we may invert the variable $T$ and then complete with respect to the norm $\max \left(||_{1},||_{r}\right)$ in order to obtain the algebra of analytic functions on the closed annulus of inner and outer radius $r$ and 1, respectively. The purpose of this section is to generalize the concept of microlocalization in such a way that we obtain a noncommutative analog of this third construction.

In fact, what we are going to do is a rather straightforward modification of the arguments and results in $[\mathrm{Spr}]$. But since we work with normed algebras instead of filtered rings and since the paper $[\mathrm{Spr}]$ is partly obscured my confusing typographical errors we include, for the benefit of the reader, complete proofs.

Having specific applications in mind we do not strive for ultimate generality. It is clear that similar ideas will work in the more general context considered in $\mathrm{vdE}$.

We fix a (usually noncommutative) unital $K$-algebra $A$. A (nonarchimedean) norm || on $A$ is called multiplicative if

$$
|1|=1 \quad \text { and } \quad|a b|=|a| \cdot|b| \quad \text { for any } a, b \in A .
$$

Let || be such a multiplicative norm. The $\operatorname{ring} A$ of course then is an integral domain. For later reference we also recall the following triviality.

Remark A.1. If $\left|a_{0}-b_{0}\right|<\left|b_{0}\right|$ and $\left|a_{1}-b_{1}\right|<\left|b_{1}\right|$ then $\left|a_{0} a_{1}-b_{0} b_{1}\right|<\left|b_{0} b_{1}\right|$.

Proof. We compute

$$
\begin{aligned}
\left|a_{0} a_{1}-b_{0} b_{1}\right| & =\left|\left(a_{0}-b_{0}\right) a_{1}+b_{0}\left(a_{1}-b_{1}\right)\right| \\
& \left.=\max \left(\left|a_{0}-b_{0}\right| \cdot\left|a_{1}\right|,\left|b_{0}\right| \cdot \mid a_{1}-b_{1}\right)\right) \\
& <\left|b_{0}\right| \cdot\left|b_{1}\right|=\left|b_{0} b_{1}\right| .
\end{aligned}
$$
kind.

In this paper we are mostly interested in norms of the following much more restricted

Definition A.2. The multiplicative norm || on $A$ is called quasi-abelian if there is a constant $0<\gamma<1$ such that

$$
|a b-b a| \leq \gamma \cdot|a b| \quad \text { for any } a, b \in A
$$

Throughout the paper we in fact fix a finite family of quasi-abelian norms ||$_{1}, \ldots,||_{m}$ on $A$. Corresponding to each norm ||$_{i}$ we introduce the function

$$
\Delta_{i}(x, y):=|s|_{i}^{-1} \cdot|t|_{i}^{-1} \cdot|a t-s b|_{i}
$$


on $A \backslash\{0\} \times A$ where $x=(s, a)$ and $y=(t, b)$. As a second input we fix a multiplicatively closed subset $S$ of $A$ (by convention this includes the requirement that $1 \in S$ but $0 \notin S$ ). The saturation $S_{i}$ of $S$ with respect to $\|_{i}$ is the set

$$
S_{i}:=\left\{a \in A:|a t-s|_{i}<|s|_{i} \text { for some } s, t \in S\right\} .
$$

Note that this definition is symmetric in that, due to the condition (qa), we have

$$
S_{i}=\left\{a \in A:|t a-s|_{i}<|s|_{i} \text { for some } s, t \in S\right\} .
$$

Lemma A.3. $S_{i}$ is a multiplicatively closed subset containing $S$.

Proof. Obviously $S \subseteq S_{i}$ and $0 \notin S_{i}$. Let $a, b \in S_{i}$ and $s, t, s^{\prime}, t^{\prime} \in S$ such that

$$
|a t-s|_{i}<|s|_{i} \quad \text { and } \quad\left|b t^{\prime}-s^{\prime}\right|_{i}<\left|s^{\prime}\right|_{i} \text {. }
$$

By Remark A.1 we then have $\left|a t b t^{\prime}-s s^{\prime}\right|_{i}<\left|s s^{\prime}\right|_{i}$. Because of

$$
\begin{aligned}
\left|a b t t^{\prime}-s s^{\prime}\right|_{i} & =\left|a b t t^{\prime}-a t b t^{\prime}+a t b t^{\prime}-s s^{\prime}\right|_{i} \\
& \leq \max \left(|a|_{i}|b t-t b|_{i}\left|t^{\prime}\right|_{i},\left|a t b t^{\prime}-s s^{\prime}\right|_{i}\right)
\end{aligned}
$$

it therefore suffices, in order to obtain $a b \in S_{i}$, to check that

$$
|a|_{i}|b t-t b|_{i}\left|t^{\prime}\right|_{i}<\left|s s^{\prime}\right|_{i}=\left|a t b t^{\prime}\right|_{i}
$$

but which is a consequence of the condition (qa).

The crucial consequence of the condition (qa) on which everything later on relies is the following "approximative" Ore condition.

Proposition A.4. For any $\epsilon>0$ and any $(s, a) \in S \times A$ we have:

i. There is a pair $(t, b) \in S \times A$ such that

$$
|a t-s b|_{i} \leq \epsilon|a|_{i}|t|_{i} \quad\left(\text { resp. }|t a-b s|_{i} \leq \epsilon|a|_{i}|t|_{i}\right)
$$

and $|s|_{i}|b|_{i} \leq|a|_{i}|t|_{i}$ for any $1 \leq i \leq m$; if $\epsilon<1$ then $|s|_{i}|b|_{i}=|a|_{i}|t|_{i}$;

ii. if in $i$. we have $a \in S$ and $\epsilon<1$ then $b \in S_{1} \cap \ldots \cap S_{m}$.

Proof. i. Put $a_{0}:=a$ and $a_{n}:=a_{n-1} s-s a_{n-1}$ for $n \geq 1$. By (qa) we have a constant $0<\gamma<1$ such that

$$
\left|a_{n}\right|_{i} \leq \gamma\left|a_{n-1}\right|_{i}|s|_{i} \quad \text { and hence } \quad\left|a_{n}\right|_{i} \leq \gamma^{n}|s|_{i}^{n}|a|_{i}
$$

for any $n \geq 0$ and any $1 \leq i \leq m$. We therefore find an $\ell \in \mathbb{N}$ such that $\left|a_{\ell}\right|_{i} \leq \epsilon|a|_{i}\left|s^{\ell}\right|_{i}$ for any $1 \leq i \leq m$. By induction with respect to $n$ one checks that

$$
a s^{n}=\sum_{j=0}^{n-1}\left(\begin{array}{l}
n \\
j
\end{array}\right) s^{n-j} a_{j}+a_{n}
$$


and

$$
a_{n}=\sum_{j=0}^{n}(-1)^{j}\left(\begin{array}{l}
n \\
j
\end{array}\right) s^{j} a s^{n-j} .
$$

We put $t:=s^{\ell}$ and $b:=\sum_{j=0}^{\ell-1}\left(\begin{array}{l}\ell \\ j\end{array}\right) s^{\ell-j-1} a_{j}$ and obtain from (47) that $a t=s b+a_{\ell}$ and hence

$$
|a t-s b|_{i} \leq \epsilon|a|_{i}|t|_{i}
$$

for any $1 \leq i \leq m$. By (48) we have $\left|a_{n}\right|_{i} \leq|s|_{i}^{n} \mid a_{i}$ for any $n \geq 0$ and therefore $|b|_{i} \leq$ $\left|s^{\ell-1}\right|_{i}|a|_{i}=|s|_{i}^{-1}|t|_{i}|a|_{i}$. The stated identity in case $\epsilon<1$ is obvious. The second half of the assertion is shown analogously. ii. This is clear.

Corollary A.5. For any $\epsilon>0$ and any $x \in S \times A$ there is a $\xi \in S \times A$ such that $\Delta_{i}(x, \xi) \leq \epsilon$ for any $1 \leq i \leq m$.

We now introduce, for any $1 \leq i \leq m$, the function

$$
d_{i}(x, y):=\inf _{\xi \in S_{i} \times A} \max \left(\Delta_{i}(x, \xi), \Delta_{i}(y, \xi)\right)
$$

on $\left(S_{i} \times A\right)^{2}$ as well as the function

$$
d(x, y):=\max \left(d_{1}(x, y), \ldots, d_{m}(x, y)\right)
$$

on $(S \times A)^{2}$. Obviously we have $d(x, y)=d(y, x) \geq 0$. Furthermore, it follows from Cor. A.5 that $d(x, x)=0$.

Proposition A.6. For any $x, y, z \in S \times A$ we have

$$
d(x, z) \leq \max (d(x, y), d(y, z)) .
$$

Proof. It certainly suffices to establish the inequality

$$
d_{i}(x, z) \leq \max \left(d_{i}(x, y), d_{i}(y, z)\right)
$$

for each individual $1 \leq i \leq m$. Given any constant $\gamma_{0}>\max \left(d_{i}(x, y), d_{i}(y, z)\right)$ we have to show that $d_{i}(x, z)<\gamma_{0}$. Let $x=(s, a), y=(t, b)$, and $z=(u, c)$. We find $\xi=(\sigma, \alpha)$ and $\eta=(\tau, \beta)$ in $S_{i} \times A$ such that $\Delta_{i}(x, \xi), \Delta_{i}(y, \xi), \Delta_{i}(y, \eta)$, and $\Delta_{i}(z, \eta)$ all are smaller than $\gamma_{0}$, i.e., such that

$$
\begin{aligned}
& |a \sigma-s \alpha|_{i}<\gamma_{0}|s|_{i}|\sigma|_{i},|b \sigma-t \alpha|_{i}<\gamma_{0}|t|_{i}|\sigma|_{i}, \\
& |b \tau-t \beta|_{i}<\gamma_{0}|t|_{i}|\tau|_{i},|c \tau-u \beta|_{i}<\gamma_{0}|u|_{i}|\tau|_{i} .
\end{aligned}
$$

We choose a $0<\epsilon<1$ such that $\epsilon|b|_{i} \leq \gamma_{0}|t|_{i}$ and $\epsilon|c|_{i} \leq \gamma_{0}|u|_{i}$. By Prop. A.4.i applied to the multiplicative set $S_{i}$ and $(\tau, \sigma) \in S_{i} \times A$ there is a pair $(v, d) \in S_{i} \times A$ such that

$$
|\sigma v-\tau d|_{i}<\epsilon|\sigma|_{i}|v|_{i}<|\sigma|_{i}|v|_{i}=|\tau|_{i}|d|_{i} .
$$

It follows that

$$
\begin{aligned}
|t \alpha v-t \beta d|_{i} & =|t \alpha v-b \sigma v+b \sigma v-b \tau d+b \tau d-t \beta d|_{i} \\
& <\max \left(\gamma_{0}|t|_{i}|\sigma|_{i}|v|_{i}, \epsilon|b|_{i}|\sigma|_{i}|v|_{i}, \gamma_{0}|t|_{i}|\tau|_{i}|d|_{i}\right) \\
& =\gamma_{0}|t|_{i}|\sigma|_{i}|v|_{i} .
\end{aligned}
$$


By the multiplicativity of ||$_{i}$ we then must have

$$
|\alpha v-\beta d|_{i}<\gamma_{0}|\sigma|_{i}|v|_{i}
$$

We therefore obtain

$$
\begin{aligned}
|s \beta d-a \sigma v|_{i} & =|s \beta d-s \alpha v+s \alpha v-a \sigma v|_{i} \\
& <\gamma_{0}|s|_{i}|\sigma|_{i}|v|_{i}=\gamma_{0}|s|_{i}|\sigma v|_{i}
\end{aligned}
$$

and

$$
\begin{aligned}
|u \beta d-c \sigma v|_{i} & =|u \beta d-c \tau d+c \tau d-c \sigma v|_{i} \\
& <\max \left(\gamma_{0}|u|_{i}|\tau|_{i}|d|_{i}, \epsilon|c|_{i}|\sigma|_{i}|v|_{i}\right) \\
& =\gamma_{0}|u|_{i}|\sigma|_{i}|v|_{i}=\gamma_{0}|u|_{i}|\sigma v|_{i} .
\end{aligned}
$$

This means that

$$
d_{i}(x, z) \leq \max \left(\Delta_{i}(x,(\sigma v, \beta d)), \Delta_{i}(z,(\sigma v, \beta d))\right)<\gamma_{0}
$$

We obtain that $d$ is a pseudometric on the set $S \times A$. The object of interest in this paper is the Hausdorff completion of the pseudometric space $(S \times A, d)$. But first we give a few explicit formulae for $d$.

Lemma A.7. For any $1 \leq i \leq m$ and any $a, b \in A$ and $s, t \in S_{i}$ we have:
i. $d_{i}((s, a),(t s, t a))=0$;
ii. $d_{i}((s, a),(s, b))=|s|_{i}^{-1}|a-b|_{i}$;
iii. $d_{i}((s, a),(1,0))=|s|_{i}^{-1}|a|_{i}$
iv. $d_{i}((s, a),(t, a)) \leq|s|_{i}^{-1}|t|_{i}^{-1}|a|_{i}|s-t|_{i}$.

Proof. i. For any $\xi \in A \backslash\{0\} \times A$ we have

$$
\Delta_{i}((t s, t a), \xi)=\Delta_{i}((s, a), \xi) .
$$

Hence $d_{i}((s, a),(t s, t a))=\inf _{\xi \in S_{i} \times A} \Delta_{i}((s, a), \xi)$ and the assertion follows from Cor. A.5 applied to $S_{i} \times A$.

ii. For $\xi=(\sigma, \alpha) \in A \backslash\{0\} \times A$ we have

$$
\begin{aligned}
\max \left(\Delta_{i}((s, a), \xi), \Delta_{i}((s, b), \xi)\right) & =|s|_{i}^{-1}|\sigma|_{i}^{-1} \max \left(|a \sigma-s \alpha|_{i},|b \sigma-s \alpha|_{i}\right) \\
& \geq|s|_{i}^{-1}|\sigma|_{i}^{-1}|a \sigma-b \sigma|_{i}=|s|_{i}^{-1}|a-b|_{i}
\end{aligned}
$$

Hence $d_{i}((s, a),(s, b)) \geq|s|_{i}^{-1}|a-b|_{i}$. On the other hand we have

$$
\begin{aligned}
\Delta_{i}((s, b), \xi) & =|s|_{i}^{-1}|\sigma|_{i}^{-1}|b \sigma-s \alpha|_{i} \\
& =|s|_{i}^{-1}|\sigma|_{i}^{-1}|b \sigma-a \sigma+a \sigma-s \alpha|_{i} \\
& \leq \max \left(|s|_{i}^{-1}|b-a|_{i}, \Delta_{i}((s, a), \xi)\right) .
\end{aligned}
$$


Using Cor. A.5 (applied to $\left.S_{i} \times A\right)$ it follows that $d_{i}((s, a),(s, b)) \leq|s|_{i}^{-1}|a-b|_{i}$.

iii. As a special case of ii. we have $d_{i}((s, a),(s, 0))=|s|_{i}^{-1}|a|_{i}$. We also have $d_{i}((1,0),(s, 0))=$

0 by i. It then follows from Prop. A.6 that $d_{i}((s, a),(1,0))=d_{i}((s, a),(s, 0))=|s|_{i}^{-1}|a|_{i}$.

iv. For $\xi=(\sigma, \alpha) \in A \backslash\{0\} \times A$ we have

$$
\begin{aligned}
\Delta_{i}((t, a), \xi) & =|t|_{i}^{-1}|\sigma|_{i}^{-1}|a \sigma-t \alpha|_{i} \\
& \leq|t|_{i}^{-1}|\sigma|_{i}^{-1} \max \left(|a \sigma-s \alpha|_{i},|s-t|_{i}|\alpha|_{i}\right) .
\end{aligned}
$$

By Prop. A.4.i there is a $\xi \in S_{i} \times A$, for any $\epsilon>0$, such that

$$
|a \sigma-s \alpha|_{i} \leq \epsilon|a|_{i}|\sigma|_{i} \quad \text { and } \quad|\alpha|_{i} \leq|s|_{i}^{-1}|a|_{i}|\sigma|_{i}
$$

For such a $\xi$ we have

$$
\Delta_{i}((t, a), \xi) \leq|t|_{i}^{-1} \max \left(\epsilon|a|_{i},|s|_{i}^{-1}|a|_{i}|s-t|_{i}\right)
$$

It follows that

$$
\begin{aligned}
d_{i}((s, a),(t, a)) & \leq \max \left(\Delta_{i}((s, a), \xi), \Delta_{i}((t, a), \xi)\right) \\
& =\max \left(|s|_{i}^{-1}|\sigma|_{i}^{-1}|a \sigma-s \alpha|_{i}, \Delta_{i}((t, a), \xi)\right) \\
& \leq \max \left(\epsilon|s|_{i}^{-1}|a|_{i}, \epsilon|t|_{i}^{-1}|a|_{i},|s|_{i}^{-1}|t|_{i}^{-1}|a|_{i}|s-t|_{i}\right)
\end{aligned}
$$

for any $\epsilon>0$ and hence $d_{i}((s, a),(t, a)) \leq|s|_{i}^{-1}|t|_{i}^{-1}|a|_{i}|s-t|_{i}$.

Let now $\mathcal{C}(S \times A)$ denote the set of all Cauchy sequences $\left(x_{j}\right)_{j \in \mathbb{N}}$ (w.r.t. $\left.d\right)$ in $S \times A$. It contains $S \times A$ via the constant sequences. The pseudometric $d$ extends to $\mathcal{C}(S \times A)$ by

$$
d\left(\left(x_{j}\right)_{j},\left(y_{j}\right)_{j}\right):=\lim _{j \rightarrow \infty} d\left(x_{j}, y_{j}\right) .
$$

We let $B:=A\left\langle S ;||_{1}, \ldots,||_{m}\right\rangle$ denote the quotient of $\mathcal{C}(S \times A)$ by the equivalence relation

$$
\left(x_{j}\right)_{j} \sim\left(y_{j}\right)_{j} \quad \text { if } \quad d\left(\left(x_{j}\right)_{j},\left(y_{j}\right)_{j}\right)=0 .
$$

The pseudometric $d$ passes to a metric on $B$ which we again denote by $d$. The metric space $(B, d)$ together with the obvious map $S \times A \longrightarrow B$ is the Hausdorff completion of the pseudometric space $(S \times A, d)$. We let $s^{-1} a \in B$ denote the image of $(s, a) \in S \times A$. Obviously

$$
s^{-1} a=t^{-1} b \quad \text { if and only if } \quad d((s, a),(t, b))=0 .
$$

By Lemma A.7,ii the composed map

$$
\begin{aligned}
\left(A, \max \left(||_{1}, \ldots,||_{m}\right)\right) & \longrightarrow(B, d) \\
a & \longmapsto 1^{-1} a=: a
\end{aligned}
$$

is an isometry.

In the following we will show that $B$ naturally is a $K$-Banach algebra. We begin with the scalar multiplication by $K$. On the set $S \times A$ we have a $K$-action given by

$$
\lambda(s, a):=(s, \lambda a)
$$


It satisfies

$$
d(\lambda x, \lambda y)=|\lambda| d(x, y)
$$

and therefore extends to a $K$-action

$$
\lambda\left(x_{j}\right)_{j}:=\left(\lambda x_{j}\right)_{j}
$$

on $\mathcal{C}(S \times A)$ which in turn descends to a $K$-action on $B$. The natural map $A \longrightarrow B$ is $K$-equivariant.

Next we construct the addition, and begin with the following immediate consequence of Lemma A.7.ii.

Remark A.8. For any $s, t \in S$ and $a_{1}, a_{2}, b_{1}, b_{2} \in A$ we have

$$
d\left(\left(s, a_{1}+a_{2}\right),\left(s, b_{1}+b_{2}\right)\right) \leq \max \left(d\left(\left(s, a_{1}\right),\left(s, b_{1}\right)\right), d\left(\left(s, a_{2}\right),\left(s, b_{2}\right)\right)\right) .
$$

Lemma A.9. Let $0<\epsilon<1$; for $s, t \in S$ and $a, b, c, d \in A$ such that

$$
d((s, a),(t, c)) \leq \epsilon \quad \text { and } \quad d((s, b),(t, d)) \leq \epsilon
$$

we have

$$
d((s, a+b),(t, c+d)) \leq \epsilon \max _{1 \leq i \leq m} \max \left(1,|s|_{i}^{-1}|a|_{i},|s|_{i}^{-1}|b|_{i},|t|_{i}^{-1}|c|_{i},|t|_{i}^{-1}|d|_{i}\right)
$$

Proof. Applying Prop. A.4 to $(t, s)$ we find $(u, v) \in S \times S_{1} \cap \ldots \cap S_{m}$ such that

$$
|u s-v t|_{i} \leq \epsilon|u|_{i}|s|_{i}<|u|_{i}|s|_{i}=|v|_{i}|t|_{i}
$$

for any $1 \leq i \leq m$. We claim that

$$
d_{i}((s, a),(u s, v c)) \leq \epsilon \max \left(1,|s|_{i}^{-1}|a|_{i}\right) .
$$

For any $\xi=(\sigma, \alpha) \in S_{i} \times A$ we have

$$
\begin{aligned}
|v c \sigma-u s \alpha|_{i} & =|v(c \sigma-t \alpha)+(v t-u s) \alpha|_{i} \\
& \leq \max \left(|t|_{i}^{-1}|u|_{i}|s|_{i}|c \sigma-t \alpha|_{i}, \epsilon|u|_{i}|s|_{i}|\alpha|_{i}\right)
\end{aligned}
$$

and hence

$$
\begin{aligned}
\Delta_{i}((u s, v c), \xi) & \leq \max \left(|t|_{i}^{-1}|\sigma|_{i}^{-1}|c \sigma-t \alpha|_{i}, \epsilon|\sigma|_{i}^{-1}|\alpha|_{i}\right) \\
& =\max \left(\Delta_{i}((t, c), \xi), \epsilon|\sigma|_{i}^{-1}|\alpha|_{i}\right)
\end{aligned}
$$

Since $d((s, a),(t, c)) \leq \epsilon$ we find a $\xi$ with $\max \left(\Delta_{i}((s, a), \xi), \Delta_{i}((t, c), \xi)\right) \leq \epsilon$. We conclude that

$$
\begin{aligned}
d_{i}((s, a),(u s, v c)) & \leq \max \left(\Delta_{i}((s, a), \xi), \Delta_{i}((u s, v c), \xi)\right) \\
& \leq \epsilon \max \left(1,|\sigma|_{i}^{-1}|\alpha|_{i}\right)
\end{aligned}
$$


But $\Delta_{i}((s, a), \xi) \leq \epsilon$ also implies that $|\sigma|_{i}^{-1}|\alpha|_{i} \leq \max \left(|s|_{i}^{-1}|a|_{i}, \epsilon\right)$. Hence our claim (observe that in case $|s|_{i}^{-1}|a|_{i}<\epsilon<1$ we have $\epsilon \max \left(1,|\sigma|_{i}^{-1}|\alpha|_{i}\right)=\epsilon=\epsilon \max \left(1,|s|_{i}^{-1}|a|_{i}\right)$. By the same reasoning we also have

$$
d_{i}((s, b),(u s, v d)) \leq \epsilon \max \left(1,|s|_{i}^{-1}|b|_{i}\right) .
$$

Applying Lemma A.7, i to the element $v \in S_{1} \cap \ldots \cap S_{m}$ we obtain

$$
\begin{aligned}
d_{i}((s, a+b),(t, c+d)) & =d_{i}((s, a+b),(v t, v(c+d))) \\
\leq & \max \left(d_{i}((s, a+b),(u s, v(c+d))), d_{i}((u s, v(c+d)),(v t, v(c+d)))\right) .
\end{aligned}
$$

Using again Lemma A.7,i and Remark A.8 we have

$$
\begin{aligned}
d_{i}((s, a+b),(u s, v(c+d)))=d_{i}((u s, u(a+b)),(u s, v(c+d))) \\
\quad \leq \max \left(d_{i}((u s, u a),(u s, v c)), d_{i}((u s, u b),(u s, v d))\right) \\
\quad=\max \left(d_{i}((s, a),(u s, v c)), d_{i}((s, b),(u s, v d))\right) \\
\quad \leq \epsilon \max \left(1,|s|_{i}^{-1}|a|_{i},|s|_{i}^{-1}|b|_{i}\right) .
\end{aligned}
$$

According to Lemma A.7,iv we have

$$
\begin{aligned}
& d_{i}((u s, v(c+d)),(v t, v(c+d))) \\
& \left.\quad \leq|u|_{i}^{-1}|s|_{i}^{-1}|v|_{i}^{-1}|t|_{i}^{-1}|v|_{i}|c+d|_{i}|u s-v t|_{i}\right) \\
& \quad \leq \epsilon|t|_{i}^{-1}|c+d|_{i} .
\end{aligned}
$$

It follows that

$$
d_{i}((s, a+b),(t, c+d)) \leq \epsilon \max \left(1,|s|_{i}^{-1}|a|_{i},|s|_{i}^{-1}|b|_{i},|t|_{i}^{-1}|c+d|_{i}\right) .
$$

This finishes the proof.

Corollary A.10. For $s, t \in S$ and $a, b, c, d \in A$ such that $s^{-1} a=t^{-1} c$ and $s^{-1} b=t^{-1} d$ we have $s^{-1}(a+b)=t^{-1}(c+d)$.

Proof. Our assumption amounts to $d((s, a),(t, c))=d((s, b),(t, d))=0$. The previous lemma then implies that $d((s, a+b),(t, c+d))=0$.

At this point we introduce, for any $n \in \mathbb{N}$, the subset

$$
\begin{array}{r}
P^{(n)}:=\left\{\left(e_{1}, \ldots, e_{n}\right) \in B^{n}: \text { there are } a_{1}, \ldots, a_{n} \in A \text { and } s \in S\right. \text { such } \\
\text { that } \left.e_{j}=s^{-1} a_{j} \text { for any } 1 \leq j \leq n\right\}
\end{array}
$$

of $B^{n}$. Of course, $P^{(1)}$ is just the image of the natural map $S \times A \longrightarrow B$. By Lemma A.7,iii the element

$$
0:=s^{-1} 0
$$

is well defined in $P^{(1)}$ (i.e., is independent of the choice of $s \in S$ ). Cor. A.10 says that the map

$$
\begin{aligned}
P^{(2)} & \longrightarrow B \\
(e, f) & \longmapsto e+f:=s^{-1}(a+b) \text { if } e=s^{-1} a, f=s^{-1} b
\end{aligned}
$$

is well defined. We obviously have: 
1. $e+f=f+e$,

2. $e+0=e$,

3. $e+(-1) e=0$,

4. $(e+f)+g=e+(f+g)$ for $(e, f, g) \in P^{(3)}$,

5. $\lambda(e+f)=\lambda e+\lambda f$ for $\lambda \in K$,

6. $(\lambda+\mu) e=\lambda e+\mu e$ for $\lambda, \mu \in K$.

For any $e \in B$ we put

$$
|e|:=d(e, 0) \text {. }
$$

It follows from Lemma A.7.ii and iii that for $(e, f) \in P^{(2)}$ we have

7. $d(e, f)=|e-f|$.

With this notation we may rephrase Lemma A.9 as follows.

Corollary A.11. Let $0<\epsilon<1$; for any $\left(e_{1}, f_{1}\right)$ and $\left(e_{2}, f_{2}\right)$ in $P^{(2)}$ such that

$$
d\left(e_{1}, e_{2}\right) \leq \epsilon \quad \text { and } \quad d\left(f_{1}, f_{2}\right) \leq \epsilon
$$

we have

$$
d\left(e_{1}+f_{1}, e_{2}+f_{2}\right) \leq \epsilon \max \left(1,\left|e_{1}\right|,\left|e_{2}\right|,\left|f_{1}\right|,\left|f_{2}\right|\right) .
$$

This corollary shows that the map $+: P^{(2)} \longrightarrow B$ is continuous and extends continuously to the closure of $P^{(2)}$ in $B^{2}$ (cf. [B-GT] II§3.6 Prop. 11 and IX $\S 2.3$ ).

Lemma A.12. $P^{(n)}$, for any $n \in \mathbb{N}$, is dense in $B^{n}$ (for the product topology).

Proof. We have to find, for any given $\epsilon>0$ and $x_{1}, \ldots, x_{n} \in S \times A$, elements $a_{1}, \ldots, a_{n} \in A$ and $s \in S$ such that

$$
d\left(x_{j},\left(s, a_{j}\right)\right)<\epsilon \quad \text { for any } 1 \leq j \leq n .
$$

We may assume $n>1$ and, by induction with respect to $n$, also that $x_{1}=\left(t, b_{1}\right), \ldots, x_{n-1}=$ $\left(t, b_{n-1}\right)$. Let $x_{n}=(u, c)$ and choose $0<\eta<1$ such that $\eta \cdot \max _{1 \leq i \leq m}|c|_{i}|u|_{i}^{-1}<\epsilon$. By Prop. A.4. $\mathrm{i}$ there are elements $(\sigma, \alpha),(\tau, \beta) \in S \times A$ such that

$$
\begin{aligned}
|\sigma t-\alpha u|_{i} & \leq \eta|\sigma|_{i}|t|_{i}<|\sigma|_{i}|t|_{i}=\left|\alpha_{i}\right|_{i}|u|_{i} \\
|c \tau-u \beta|_{i} & \leq \eta|c|_{i}|\tau|_{i} \leq|c|_{i}|\tau|_{i}=|u|_{i}|\beta|_{i}
\end{aligned}
$$

for any $1 \leq i \leq m$. In particular

$$
\begin{aligned}
|\alpha c \tau-\sigma t \beta|_{i} & \leq \max \left(|\alpha|_{i}|c \tau-u \beta|_{i},|\alpha u-\sigma t|_{i}|\beta|_{i}\right) \\
& \leq \eta \max \left(|\alpha|_{i}|c|_{i}|\tau|_{i},|\beta|_{i}|\sigma|_{i}|t|_{i}\right) \\
& =\eta|u|_{i}^{-1}|c|_{i}|\tau|_{i}|\sigma|_{i}|t|_{i} .
\end{aligned}
$$


We put $y_{j}:=\left(\sigma t, \sigma b_{j}\right)$ for $1 \leq j \leq n-1$ and $y_{n}:=(\sigma t, \alpha c)$. According to Lemma A.7,i we have

$$
d\left(x_{j}, y_{j}\right)=0 \quad \text { for any } 1 \leq j \leq n-1
$$

Furthermore

$$
\begin{aligned}
d_{i}\left(x_{n}, y_{n}\right) & \leq \max \left(\Delta_{i}\left(x_{n},(\tau, \beta)\right), \Delta_{i}\left(y_{n},(\tau, \beta)\right)\right) \\
& =\max \left(|u|_{i}^{-1}|\tau|_{i}^{-1}|c \tau-u \beta|_{i},|\sigma|_{i}^{-1}|t|_{i}^{-1}|\tau|_{i}^{-1}|\alpha c \tau-\sigma t \beta|_{i}\right) \\
& \leq \eta|c|_{i}|u|_{i}^{-1}<\epsilon
\end{aligned}
$$

It follows in particular that our map $+: P^{(2)} \longrightarrow B$ extends continuously to a map

$$
\begin{aligned}
B \times B & \longrightarrow B \\
(e, f) & \longmapsto e+f
\end{aligned}
$$

which satisfies 1.-7. (for the associativity use Lemma A.12 for $n=3$ ). We see that || is a nonarchimedean norm which makes $B$ into a $K$-Banach space. The natural map

$$
\left(A, \max \left(||_{1}, \ldots,||_{m}\right)\right) \longrightarrow(B,||)
$$

is an isometry of normed $K$-vector spaces.

In order to construct the multiplication on $B$ we proceed in a similar way.

Lemma A.13. Let $0<\epsilon<1$; for $s, t, u, v \in S$ and $a, b, c, d \in A$ such that

$$
d((s, a t),(u, c v)) \leq \epsilon \quad \text { and } \quad d((t, b),(v, d)) \leq \epsilon
$$

we have

$$
d((s, a b),(u, c d)) \leq \epsilon \max _{1 \leq i \leq m} \max \left(1, \frac{|a t|_{i}}{|s|_{i}}, \frac{|a t|_{i}}{|s|_{i}} \frac{|b|_{i}}{|t|_{i}}, \frac{|b|_{i}}{|t|_{i}}, \frac{|c v|_{i}}{|u|_{i}}, \frac{|c v|_{i}}{|u|_{i}} \frac{|d|_{i}}{|v|_{i}}, \frac{|d|_{i}}{|v|_{i}}\right)
$$

Proof. Let $0 \leq i \leq m$. We find $\xi=(\sigma, \alpha)$ and $\eta=(\tau, \beta)$ in $S_{i} \times A$ such that

$$
\begin{gathered}
|b \sigma-t \alpha|_{i} \leq \epsilon|\sigma|_{i}|t|_{i}, \quad|d \sigma-v \alpha|_{i} \leq \epsilon|\sigma|_{i}|v|_{i}, \\
|a t \tau-s \beta|_{i} \leq \epsilon|\tau|_{i}|s|_{i}, \quad|c v \tau-u \beta|_{i} \leq \epsilon|\tau|_{i}|u|_{i} .
\end{gathered}
$$

In particular, we have $|\sigma|_{i}^{-1}|\alpha|_{i} \leq \max \left(|t|_{i}^{-1}|b|_{i}, \epsilon\right) \leq \max \left(|t|_{i}^{-1}|b|_{i}, 1\right)$ and also $|\sigma|_{i}^{-1}|\alpha|_{i} \leq$ $\max \left(|v|_{i}^{-1}|d|_{i}, 1\right)$. Using Prop. A.4.i (for $\left.S_{i}\right)$ we choose $(\rho, \kappa) \in S_{i} \times A$ such that

$$
|\alpha \rho-\tau \kappa|_{i} \leq \epsilon|\alpha|_{i}|\rho|_{i},|\tau|_{i}|\kappa|_{i}=|\alpha|_{i}|\rho|_{i}
$$

We then have

$$
\begin{aligned}
|a b \sigma \rho-s \beta \kappa|_{i} & =|a(b \sigma-t \alpha) \rho+a t(\alpha \rho-\tau \kappa)+(a t \tau-s \beta) \kappa|_{i} \\
& \leq \epsilon \max \left(|a|_{i}|\sigma|_{i}|t|_{i}|\rho|_{i},|a|_{i}|t|_{i}|\alpha|_{i}|\rho|_{i},|s|_{i}|\alpha|_{i}|\rho|_{i}\right)
\end{aligned}
$$


and hence

$$
\begin{aligned}
\Delta_{i}((s, a b),(\sigma \rho, \beta \kappa)) & \leq \epsilon \max \left(|s|_{i}^{-1}|a t|_{i},|s|_{i}^{-1}|a t|_{i}|\sigma|_{i}^{-1}|\alpha|_{i},|\sigma|_{i}^{-1}|\alpha|_{i}\right) \\
& \leq \epsilon \max \left(|s|_{i}^{-1}|a t|_{i},|s|_{i}^{-1}|a t|_{i}|t|_{i}^{-1}|b|_{i},|t|_{i}^{-1}|b|_{i}, 1\right)
\end{aligned}
$$

Similarly

$$
\begin{aligned}
|c d \sigma \rho-u \beta \kappa|_{i} & =|c(d \sigma-v \alpha) \rho+c v(\alpha \rho-\tau \kappa)+(c v \tau-u \beta) \kappa|_{i} \\
& \leq \epsilon \max \left(|c|_{i}|\sigma|_{i}|v|_{i}|\rho|_{i},|c|_{i}|v|_{i}|\alpha|_{i}|\rho|_{i},|u|_{i}|\alpha|_{i}|\rho|_{i}\right)
\end{aligned}
$$

and

$$
\begin{aligned}
\Delta_{i}((u, c d),(\sigma \rho, \beta \kappa)) & \leq \epsilon \max \left(|u|_{i}^{-1}|c v|_{i},|u|_{i}^{-1}|c v|_{i}|\sigma|_{i}^{-1}|\alpha|_{i},|\sigma|_{i}^{-1}|\alpha|_{i}\right) \\
& \leq \epsilon \max \left(|u|_{i}^{-1}|c v|_{i},|u|_{i}^{-1}|c v|_{i}|v|_{i}^{-1}|d|_{i},|v|_{i}^{-1}|d|_{i}, 1\right) .
\end{aligned}
$$

We obtain

$$
d_{i}((s, a b),(u, c d)) \leq \epsilon \max \left(1, \frac{|a t|_{i}}{|s|_{i}}, \frac{|a t|_{i}}{|s|_{i}} \frac{|b|_{i}}{|t|_{i}}, \frac{|b|_{i}}{|t|_{i}}, \frac{|c v|_{i}}{|u|_{i}}, \frac{|c v|_{i}}{|u|_{i}} \frac{|d|_{i}}{|v|_{i}}, \frac{|d|_{i}}{|v|_{i}}\right) .
$$

Corollary A.14. For $s, t, u, v \in S$ and $a, b, c, d \in A$ such that $s^{-1}(a t)=u^{-1}(c v)$ and $t^{-1} b=$ $v^{-1} d$ we have $s^{-1}(a b)=u^{-1}(c d)$.

This corollary says that on the subset

$$
Q:=\left\{\left(s^{-1} a, t^{-1} b\right) \in\left(P^{(1)}\right)^{2}: a \in A t\right\}
$$

of $B^{2}$ the map

$$
\begin{aligned}
Q & \longrightarrow B \\
(e, f) & \longmapsto e \cdot f:=s^{-1}(a b) \text { if } e=s^{-1}(a t), f=t^{-1} b
\end{aligned}
$$

is well defined.

Corollary A.15. Let $0<\epsilon<1$; for any $\left(e_{1}, f_{1}\right)$ and $\left(e_{2}, f_{2}\right)$ in $Q$ such that

$$
d\left(e_{1}, e_{2}\right) \leq \epsilon \quad \text { and } \quad d\left(f_{1}, f_{2}\right) \leq \epsilon
$$

we have

$$
d\left(e_{1} \cdot f_{1}, e_{2} \cdot f_{2}\right) \leq \epsilon \max \left(1,\left|e_{1}\right|,\left|e_{1}\right|\left|f_{1}\right|,\left|f_{1}\right|,\left|e_{2}\right|,\left|e_{2}\right|\left|f_{2}\right|,\left|f_{2}\right|\right) .
$$

This corollary shows that the map $\cdot: Q \longrightarrow B$ is continuous and extends continuously to the closure of $Q$ in $B^{2}$.

Lemma A.16. For any given $t \in S$ the set $\left\{s^{-1}(a t): a \in A, s \in S\right\}$ is dense in $B$. 
Proof. Let $(u, c) \in S \times A$ and let $\epsilon>0$. By Prop. A.4.i we find $(\sigma, \alpha) \in S \times A$ such that $|\sigma c-\alpha t|_{i} \leq \epsilon|\sigma|_{i}|c|_{i}$ for any $1 \leq i \leq m$. Using Lemma A.7,i and ii we obtain

$$
\begin{aligned}
d\left(u^{-1} c,(\sigma u)^{-1}(\alpha t)\right) & =d\left((\sigma u)^{-1}(\sigma c),(\sigma u)^{-1}(\alpha t)\right) \\
& =\max _{1 \leq i \leq m}|u|_{i}^{-1}|\sigma|_{i}^{-1}|\sigma c-\alpha t|_{i} \\
& \leq \epsilon \max _{1 \leq i \leq m}|u|_{i}^{-1}|c|_{i}
\end{aligned}
$$

This lemma implies that $Q$ is dense in $B^{2}$. Hence by continuous extension we have a map

$$
\begin{aligned}
B \times B & \longrightarrow B \\
(e, f) & \longmapsto e \cdot f .
\end{aligned}
$$

Lemma A.17. For any $e, f, g \in B$ we have:

$$
\begin{aligned}
& \text { i. } d(e \cdot f, e \cdot g) \leq|e| d(f, g) \text { and } e \cdot(f+g)=e \cdot f+e \cdot g ; \\
& \text { ii. } d(e \cdot g, f \cdot g) \leq d(e, f)|g| \text { and }(e+f) \cdot g=e \cdot g+f \cdot g ; \\
& \text { iii. }(e \cdot f) \cdot g=e \cdot(f \cdot g) \text {. }
\end{aligned}
$$

Proof. By continuity all three assertions need only to be checked on an appropriate dense subset of $B^{3}$.

i. As a consequence of Lemma A.12 (for $n=2$ ) and Lemma A.16 the set

$$
\left\{\left(s^{-1}(a t), t^{-1} b, t^{-1} c\right) \in\left(P^{(1)}\right)^{3}: a, b, c \in A, s, t \in S\right\}
$$

is dense in $B^{3}$. For a triple in this set the first inequality is immediate from Lemma A.7.ii and iii and the second identity follows from the definitions.

ii. As a consequence of Lemma A.16 the set $\left\{\left(s_{1}^{-1}\left(a^{\prime} t\right), s_{2}^{-1}\left(b^{\prime} t\right), t^{-1} c\right)\right\}$ is dense in $B^{3}$. In addition, the proof of Lemma A.12 (for $n=2$ ) shows that a pair $\left(s_{1}^{-1}\left(a^{\prime} t\right), s_{2}^{-1}\left(b^{\prime} t\right)\right)$ can be approximated by a pair of the form $\left(s^{-1}(a t), s^{-1}(b t)\right)$. Hence the set

$$
\left\{\left(s^{-1}(a t), s^{-1}(b t), t^{-1} c\right) \in\left(P^{(1)}\right)^{3}: a, b, c \in A, s, t \in S\right\}
$$

is dense in $B^{3}$. For a triple in this set the first inequality again is immediate from Lemma A.7.ii and iii and the second identity again follows from the definitions.

iii. By Lemma A.16 the set

$$
\left\{\left(s^{-1}(a t), t^{-1}(b u), u^{-1} c\right) \in\left(P^{(1)}\right)^{3}: a, b, c \in A, s, t, u \in S\right\}
$$

is dense in $B^{3}$. On this subset the asserted associativity is immediate from the definition of the multiplication.

We see that the multiplication - in $B$ is distributive and associative. It is easy to see, using Lemma A.7,i and the density of $Q$, that 1 is a unit element and that this multiplication 
is compatible with the scalar multiplication by $K$. Finally, $|1|=1$ and as a consequence of Lemma A.171.16.i we have $|e \cdot f| \leq|e||f|$. Hence we conclude that

$B$ is a unital $K$-Banach algebra with submultiplicative norm | |

and that the natural map $\left(A, \max \left(||_{1}, \ldots,||_{m}\right)\right) \longrightarrow(B,||)$ is an isometric unital homomorphism of normed $K$-algebras. By construction we have $\left(s^{-1} 1\right) \cdot s=s^{-1} s=1$ and $s \cdot\left(s^{-1} a\right)=a$ for any $a \in A$ and $s \in S$. In particular, the elements of the multiplicative set $S$ become invertible in the ring $B$.

Proposition A.18 (Universal property). Let $\left(D,||_{D}\right)$ be a unital $K$-Banach algebra and let $\phi: A \longrightarrow D$ be any unital homomorphism of $K$-algebras such that:

(i) $\phi(s) \in D^{\times}$for any $s \in S$;

(ii) there is a constant $\gamma>0$ such that $\left|\phi(s)^{-1} \phi(a)\right|_{D} \leq \gamma \max _{1 \leq i \leq m}|s|_{i}^{-1}|a|_{i}$ for any $s \in$ $S, a \in A$ (in particular, $\phi$ is continuous);

then there is a unique continuous unital homomorphism of $K$-Banach algebras

$$
\phi_{S}: A\left\langle S ;||_{1}, \ldots,||_{m}\right\rangle \longrightarrow D
$$

such that $\phi_{S} \mid A=\phi$. If ||$_{D}$ is submultiplicative and the constant in (ii) can be chosen to be $\gamma=1$ then $\phi_{S}$ is norm decreasing.

Proof. The subset $P^{(1)}=\left\{s^{-1} a: a \in A, s \in S\right\}$ is dense in $B$. Because of $s \cdot\left(s^{-1} a\right)=a$ we have to have $\phi_{S}\left(s^{-1} a\right)=\phi(s)^{-1} \phi(a)$. Hence the uniqueness of $\phi_{S}$ is clear. To establish existence let $\gamma_{0}>0$ be a constant such that $\left|d_{1} d_{2}\right|_{D} \leq \gamma_{0}\left|d_{1}\right|_{D}\left|d_{2}\right|_{D}$ for any $d_{1}, d_{2} \in D$. We claim that there is a constant $\gamma_{1}>0$ such that

$$
\left|\phi(s)^{-1} \phi(a)-\phi(t)^{-1} \phi(b)\right|_{D} \leq \gamma_{1}\left|s^{-1} a-t^{-1} b\right|
$$

for any $(s, a),(t, b) \in S \times A$. Choose $\eta>0$ such that $\eta|b| \leq \eta|t|\left|t^{-1}\right||b| \leq\left|s^{-1}\right|^{-1}\left|s^{-1} a-t^{-1} b\right|$. Since $P^{(1)}$ is dense in $B$ we find $(\sigma, \alpha) \in S \times A$ such that

$$
\left|s t^{-1}-\sigma^{-1} \alpha\right| \leq \eta \text {. }
$$

We then have

$$
(\sigma s)^{-1}(\sigma a-\alpha b)-(\sigma s)^{-1}(\sigma s-\alpha t) t^{-1} b=s^{-1} a-t^{-1} b
$$

and

$$
\left|(\sigma s)^{-1}(\sigma s-\alpha t) t^{-1} b\right| \leq\left|s^{-1}\right| \eta|b| \leq\left|s^{-1} a-t^{-1} b\right| .
$$

It follows that $\left|(\sigma s)^{-1}(\sigma a-\alpha b)\right| \leq\left|s^{-1} a-t^{-1} b\right|$ and hence, by (ii), that

$$
\left|\phi(\sigma s)^{-1} \phi(\sigma a-\alpha b)\right|_{D} \leq \gamma\left|s^{-1} a-t^{-1} b\right| \text {. }
$$

On the other hand

$$
\begin{aligned}
&\left|\phi(\sigma s)^{-1} \phi(\sigma s-\alpha t) \phi(t)^{-1} \phi(b)\right|_{D} \\
& \leq \gamma_{0}\left|\phi(\sigma s)^{-1} \phi(\sigma s-\alpha t)\right|_{D}\left|\phi(t)^{-1} \phi(b)\right|_{D} \\
& \leq \gamma_{0} \gamma^{2}\left|(\sigma s)^{-1}(\sigma s-\alpha t)\right|\left|t^{-1} b\right| \\
& \leq \gamma_{0} \gamma^{2}\left|(\sigma s)^{-1}(\sigma s-\alpha t) t^{-1}\right||t|\left|t^{-1}\right||b| \\
& \leq \gamma_{0} \gamma^{2}\left|s^{-1}\right| \eta|t|\left|t^{-1}\right||b| \\
& \leq \gamma_{0} \gamma^{2}\left|s^{-1} a-t^{-1} b\right| .
\end{aligned}
$$


Setting $\gamma_{1}:=\max \left(\gamma, \gamma_{0} \gamma^{2}\right)$ we finally obtain

$$
\begin{aligned}
\left|\phi(s)^{-1} \phi(a)-\phi(t)^{-1} \phi(b)\right|_{D} \\
\quad=\left|\phi(\sigma s)^{-1} \phi(\sigma a-\alpha b)-\phi(\sigma s)^{-1} \phi(\sigma s-\alpha t) \phi(t)^{-1} \phi(b)\right|_{D} \\
\quad \leq \max \left(\gamma\left|s^{-1} a-t^{-1} b\right|, \gamma_{0} \gamma^{2}\left|s^{-1} a-t^{-1} b\right|\right) \\
\quad=\gamma_{1}\left|s^{-1} a-t^{-1} b\right| .
\end{aligned}
$$

This proves our claim and implies that the map

$$
\begin{aligned}
S \times A & \longrightarrow D \\
(s, a) & \longmapsto \phi(s)^{-1} \phi(a)
\end{aligned}
$$

is uniformly continuous and therefore extends continuously to a map $\phi_{S}: B \longrightarrow D$. It is straightforward to check that $\phi_{S}$ is a unital homomorphism of $K$-algebras such that $\phi_{S} \mid A=$ $\phi$.

Although the norm || on $B$ is only submultiplicative it nevertheless is quasi-abelian in the following sense. We fix a constant $0<\gamma<1$ so that the condition (qa) is satisfies simultaneously for any ||$_{i}$.

Lemma A.19. $\left|u^{-1} s^{-1} e c-u^{-1} e s^{-1} c\right| \leq \gamma \cdot\left|u^{-1} s^{-1} e c\right|=\gamma \cdot\left|u^{-1} e s^{-1} c\right|$ for any $u, s \in S, c \in A$, and $e \in B$.

Proof. By Lemma A.16 we may assume that $e=t^{-1}(b s)$ with $t \in S$ and $b \in A$. We then compute

$$
\begin{aligned}
&\left|u^{-1} s^{-1} t^{-1} b s c-u^{-1} t^{-1} b c\right| \\
&=\left|u^{-1} s^{-1} t^{-1} b s c-u^{-1} t^{-1} s^{-1} b s c+u^{-1} t^{-1} s^{-1} b s c-u^{-1} t^{-1} s^{-1} s b c\right| \\
& \leq \max (d((t s u, b s c),(s t u, b s c)), d((s t u, b s c),(s t u, s b c))) \\
&=\max _{i} \max \left(|t s u|_{i}^{-1}|s t u|_{i}|b s c|_{i}|t s u-s t u|_{i},|s t u|_{i}^{-1}|b s c-s b c|_{i}\right) \\
& \leq \gamma \cdot \max _{i}|s t u|_{i}^{-1}|b s c|_{i}=\gamma \cdot \max _{i}|t u|_{i}^{-1}|b c|_{i} \\
&=\gamma \cdot\left|u^{-1} t^{-1} b c\right|
\end{aligned}
$$

using Lemma A.7 in the fourth line.

Lemma A.20. $\left|s^{-1} a e c-s^{-1} e a c\right| \leq \gamma \cdot\left|s^{-1} a e c\right|=\gamma \cdot\left|s^{-1} e a c\right|$ for any $s \in S, a, c \in A$, and $e \in B$.

Proof. By density we may assume that $e=t^{-1} b$ with $t \in S$ and $b \in A$. We then compute

$$
\begin{aligned}
\left|s^{-1} a t^{-1} b c-s^{-1} t^{-1} b a c\right| \\
\quad=\left|s^{-1} a t^{-1} b c-s^{-1} t^{-1} a b c+s^{-1} t^{-1} a b c-s^{-1} t^{-1} b a c\right| \\
\quad \leq \max \left(\left|s^{-1} a t^{-1} b c-s^{-1} t^{-1} a b c\right|,\left|s^{-1} t^{-1} a b c-s^{-1} t^{-1} b a c\right|\right) \\
\quad \leq \max \left(\gamma \cdot\left|s^{-1} t^{-1} a b c\right|, d((t s, a b c),(t s, b a c))\right) \\
\quad=\max \left(\gamma \cdot\left|s^{-1} t^{-1} a b c\right|, \max _{i}|t s|_{i}^{-1}|a b c-b a c|_{i}\right) \\
\quad \leq \gamma \cdot \max \left(\left|s^{-1} t^{-1} a b c\right|, \max _{i}|t s|_{i}^{-1}|a b c|_{i}\right) \\
\quad=\gamma \cdot\left|s^{-1} t^{-1} a b c\right| \\
\quad=\gamma \cdot\left|s^{-1} a t^{-1} b c\right|
\end{aligned}
$$


using the previous lemma in the fourth and the last line.

Proposition A.21. There is a constant $0<\gamma<1$ such that, for any elements $e_{1}, \ldots, e_{n} \in B$ and any permutation $\sigma$ of $\{1, \ldots, n\}$, we have

$$
\left|e_{1} \cdot \ldots \cdot e_{n}-e_{\sigma(1)} \cdot \ldots \cdot e_{\sigma(n)}\right| \leq \gamma \cdot\left|e_{1} \cdot \ldots \cdot e_{n}\right|=\gamma \cdot\left|e_{\sigma(1)} \cdot \ldots \cdot e_{\sigma(n)}\right| .
$$

Proof. Since any permutation is a product of elementary transpositions it suffices to prove that

$$
\left|g e_{0} e_{1} f-g e_{1} e_{0} f\right| \leq \gamma \cdot\left|g e_{0} e_{1} f\right|=\gamma \cdot\left|g e_{1} e_{0} f\right|
$$

for any $g, e_{0}, e_{1}, f \in B$. Since

$$
\begin{aligned}
g e_{0} e_{1} f-g e_{1} e_{0} f= & g\left(e_{0} e_{1} f\right)-\left(e_{0} e_{1} f\right) g+e_{0} e_{1}(f g)-e_{1} e_{0}(f g) \\
& +\left(e_{1} e_{0} f\right) g-g\left(e_{1} e_{0} f\right)
\end{aligned}
$$

we may in fact assume that $g=1$. By density it furthermore suffices to consider the case $f=u^{-1} c$ with $u \in S$ and $c \in A$. Using the identity

$$
\begin{aligned}
e_{0} e_{1} u^{-1} c-e_{1} e_{0} u^{-1} c= & \left(e_{0} e_{1}\right) u^{-1} c-u^{-1}\left(e_{0} e_{1}\right) c+u^{-1} e_{0} e_{1} c-u^{-1} e_{1} e_{0} c \\
& +u^{-1}\left(e_{1} e_{0}\right) c-e_{1} e_{0} u^{-1} c
\end{aligned}
$$

we therefore are reduced to showing that

$$
\left|u^{-1} e_{0} e_{1} c-u^{-1} e_{1} e_{0} c\right| \leq \gamma \cdot\left|u^{-1} e_{0} e_{1} c\right|=\gamma \cdot\left|u^{-1} e_{1} e_{0} c\right|
$$

for any $u \in S, c \in A$, and $e_{0}, e_{1} \in B$. But for $e_{0}=s^{-1} a$ with $s \in S$ and $a \in A$ this is a consequence of Lemmas A.19 and A.20. The case of a general $e_{0}$ then follows by density.

Remark A.22. For a single initial quasi-abelian norm on $A$ (i.e., $m=1$ ) the resulting norm | | on B again is multiplicative (and quasi-abelian).

Proof. We need to show that $|e f|=|e||f|$. By continuity it suffices to consider the case $e=s^{-1} a$ and $f=t^{-1} b$ with $s, t \in S$ and $a, b \in A$. But then, using Prop. A.21 and Lemma A.7.iii, we compute

$$
\left|s^{-1} a t^{-1} b\right|=\left|(t s)^{-1} a b\right|=|t s|_{1}^{-1}|a b|_{1}=|s|_{1}^{-1}|a|_{1}|t|_{1}^{-1}|b|_{1}=\left|s^{-1} a\right|\left|t^{-1} b\right| .
$$

\section{A.2 Noncommutative annuli for uniform pro- $p$-groups}

For the rest of this paper we assume that $\mathbb{Q}_{p} \subseteq K \subseteq \mathbb{C}_{p}$ is discretely valued. For simplicity we also assume that $p \neq 2$. We fix a uniform pro- $p$-group $G$ as well as an ordered basis $h_{1}, \ldots, h_{d}$ of $G$. Then the map

$$
\begin{aligned}
& \psi: \quad \mathbb{Z}_{p}^{d} \stackrel{\sim}{\longrightarrow} G \\
&\left(x_{1}, \ldots x_{d}\right) \longmapsto h_{1}^{x_{1}} \cdot \ldots \cdot h_{d}^{x_{d}}
\end{aligned}
$$


is a bijective global chart for $G$ as a locally $\mathbb{Q}_{p}$-analytic manifold (cf. [DDMS] $§ 4.2$ ). Using this chart we may identify the $K$-Fréchet spaces of locally analytic distributions

$$
\psi^{*}: D(G, K) \stackrel{\cong}{\longrightarrow} D\left(\mathbb{Z}_{p}^{d}, K\right)
$$

As usual, we embed the group $G$ into the algebra $D(G, K)$ via the Dirac distributions. We write $b_{i}:=h_{i}-1$ and $\mathbf{b}^{\alpha}:=b_{1}^{\alpha_{1}} b_{2}^{\alpha_{2}} \cdots b_{d}^{\alpha_{d}}$, for $\alpha=\left(\alpha_{1}, \ldots, \alpha_{d}\right) \in \mathbb{N}_{0}^{d}$. Any distribution $\mu \in D(G, K)$ has a unique convergent expansion

$$
\mu=\sum_{\alpha \in \mathbb{N}_{0}^{d}} d_{\alpha} \mathbf{b}^{\alpha}
$$

with $d_{\alpha} \in K$ such that, for any $0<r<1$, the set $\left\{\left|d_{\alpha}\right| r^{\alpha}\right\}_{\alpha \in \mathbb{N}_{0}^{d}}$ is bounded. Here we put $r^{\alpha}:=r^{\alpha_{1}+\ldots+\alpha_{d}}$. Conversely, any such series is convergent in $D(G, K)$. The Fréchet topology on $D(G, K)$ is defined by the family of norms

$$
|\mu|_{r}:=\sup _{\alpha \in \mathbb{N}_{0}^{d}}\left|d_{\alpha}\right| r^{\alpha}
$$

for $0<r<1$. It is shown in [ST5] Thm. 4.5 that, for any $1 / p<r<1$ such that $r \in$ $p^{\mathbb{Q}}$, the norm ||$_{r}$ on $D(G, K)$ is multiplicative and quasi-abelian. Whereas $D(G, K)$ is a "noncommutative open unit disk" the completion $D_{r}(G, K)$ of $D(G, K)$ with respect to ||$_{r}$ is a "noncommutative closed disk of radius $r$ ". We now use the technique of the previous section to construct corresponding "noncommutative closed annuli" for any radii $1 / p<r_{0} \leq r<1$ such that $r_{0}, r \in p^{\mathbb{Q}}$.

We take $A:=D_{r}(G, K)$, the two norms ||$_{r_{0}}$ and ||$_{r}$, and the multiplicatively closed subset $S \subseteq D_{r}(G, K)$ generated by $b_{1}, \ldots, b_{d}$. We define

$$
D_{\left[r_{0}, r\right]}(G, K):=A\left\langle S ;||_{r_{0}},||_{r}\right\rangle
$$

and we let ||$_{r_{0}, r}$ denote its natural norm. Since $\max \left(||_{r_{0}},||_{r}\right)=||_{r}$ the canonical homomorphism of $K$-Banach algebras $D_{r}(G, K) \longrightarrow D_{\left[r_{0}, r\right]}(G, K)$ is an isometry for the natural norms.

Lemma A.23. For $1 / p<r \leq r^{\prime} \leq r^{\prime \prime}<1$, any integer $m \geq 0$, and any $\mu \in D(G, K)$ we have

$$
\frac{|\mu|_{r^{\prime}}}{r^{\prime m}} \leq \max \left(\frac{|\mu|_{r}}{r^{m}}, \frac{|\mu|_{r^{\prime \prime}}}{r^{\prime \prime m}}\right) .
$$

Proof. Since exponential function and logarithm are monotonous functions it suffices to show that the function

$$
t \longmapsto \log \left(\frac{|\mu|_{\exp (t)}}{\exp (t)^{m}}\right)
$$

on $(-\infty, 0)$ is convex. But it is a supremum

$$
t \longmapsto \sup _{\alpha}\left(\log \left|d_{\alpha}\right|+(|\alpha|-m) t\right)
$$

of affine functions and hence is visibly convex. 
Let $1 / p \leq r_{0}<r \leq r^{\prime} \leq r^{\prime \prime}<r_{1} \leq 1$ all contained in $p^{\mathbb{Q}}$ and consider the composed unital homomorphism of $K$-Banach algebras

$$
D_{r^{\prime \prime}}(G, K) \longrightarrow D_{r}(G, K) \longrightarrow D_{\left[r, r^{\prime}\right]}(G, K)
$$

which is norm decreasing and makes the elements in $S$ invertible. Let $\mu \in D_{r^{\prime}}(G, K)$ and suppose that the monomial $s$ in the $b_{1}, \ldots, b_{d}$ has $m$ factors. Using Lemma A.23 we compute

$$
\begin{aligned}
\left|s^{-1} \mu\right|_{r, r^{\prime}} & =\max \left(|s|_{r}^{-1}|\mu|_{r},|s|_{r^{\prime}}^{-1}|\mu|_{r^{\prime}}\right)=\max \left(\frac{|\mu|_{r}}{r^{m}}, \frac{|\mu|_{r^{\prime}}}{r^{\prime m}}\right) \\
& \leq \max \left(\frac{|\mu|_{r}}{r^{m}}, \frac{|\mu|_{r^{\prime \prime}}}{r^{\prime \prime m}}\right)=\max \left(|s|_{r}^{-1}|\mu|_{r},|s|_{r^{\prime \prime}}^{-1}|\mu|_{r^{\prime \prime}}\right) .
\end{aligned}
$$

This shows that the assumptions of the universal property Prop. A.18 are satisfied. The above composed homomorphism extends uniquely to a norm decreasing unital homomorphism of $K$ Banach algebras

$$
D_{\left[r, r^{\prime \prime}\right]}(G, K) \longrightarrow D_{\left[r, r^{\prime}\right]}(G, K) \text {. }
$$

We then may pass to the projective limit with respect to $r^{\prime \prime}$ and obtain the $K$-Fréchet algebra

$$
D_{\left[r, r_{1}\right)}(G, K):=\lim _{r \leq r^{\prime \prime}<r_{1}} D_{\left[r, r^{\prime \prime}\right]}(G, K)
$$

representing a "noncommutative half open annulus".

A similar argument shows that the natural homomorphism $D_{r^{\prime \prime}}(G, K) \longrightarrow D_{\left[r^{\prime}, r^{\prime \prime}\right]}(G, K)$ extends uniquely to a norm decreasing unital homomorphism of $K$-Banach algebras

$$
D_{\left[r, r^{\prime \prime}\right]}(G, K) \longrightarrow D_{\left[r^{\prime}, r^{\prime \prime}\right]}(G, K)
$$

Again we obtain a $K$-Fréchet algebra

$$
D_{\left(r_{0}, r^{\prime \prime}\right]}(G, K):=\lim _{r_{0}<r \leq r^{\prime \prime}} D_{\left[r, r^{\prime \prime}\right]}(G, K)
$$

It also follows that the Fréchet algebras $D_{\left[r, r_{1}\right)}(G, K)$ form an inductive system with respect to $r$. Especially in the case $r_{1}=1$ the inductive limit

$$
R(G, K):=\lim _{\longrightarrow} D_{[r, 1)}(G, K)
$$

is a locally convex unital $K$-algebra which we call the Robba ring of $G$. Although we suppress this in the notation this ring does depend on the initial choice of a basis $\left\{h_{1}, \ldots, h_{d}\right\}$ of $G$ but not on its ordering. It is shown in [ST5] in the discussion following Thm. 4.10 that, for $1 / p \leq r<1$, the norm \|\|$_{r}$ on $D(G, K)$ is completely independent of the choice of the ordered basis. It follows that each $D_{\left[r, r^{\prime}\right]}(G, K)$ together with its norm \|\|$_{r, r^{\prime}}$ as well as the topological algebras $D_{\left[r, r_{1}\right)}(G, K), D_{\left(r_{0}, r^{\prime \prime}\right]}(G, K)$, and $R(G, K)$ do not depend on the ordering of the chosen basis of $G$. In fact a little more is true. In the ring $\mathbb{Z}_{p}[[Z]]$ of formal power series in one variable over $\mathbb{Z}_{p}$ we have, for any $x \in \mathbb{Z}_{p}$, the identity

$$
(1+Z)^{x}-1=\sum_{i \geq 1}\left(\begin{array}{l}
x \\
i
\end{array}\right) Z^{i}=Z\left(x+Z f_{x}(Z)\right) \quad \text { with } f_{x} \in \mathbb{Z}_{p}[[Z]]
$$


If $x \in \mathbb{Z}_{p}^{\times}$then $x+Z f_{x}(Z)$ is a unit in $\mathbb{Z}_{p}[[Z]]$. This shows that for $x \in \mathbb{Z}_{p}^{\times}$we have $h_{i}^{x}-1 \in b_{i} \cdot D(G, K)^{\times}$for any $1 \leq i \leq d$. Applying the universal property Prop. A.18 we conclude that replacing $\left\{h_{1}, \ldots, h_{d}\right\}$ by $\left\{h_{1}^{x_{1}}, \ldots, h_{d}^{x_{d}}\right\}$ for some $x_{1}, \ldots, x_{d} \in \mathbb{Z}_{p}^{\times}$does not change the Banach algebras $D_{\left[r, r^{\prime}\right]}(G, K)$ together with their norm \|\|$_{r, r^{\prime}}$ and hence does not change the topological algebras $D_{\left[r, r_{1}\right)}(G, K), D_{\left(r_{0}, r^{\prime \prime}\right]}(G, K)$, and $R(G, K)$ either.

In order to be able to work with these rings we will show that their elements can be viewed as Laurent series. For that we introduce the affinoid domain

$$
X_{\left[r, r^{\prime}\right]}^{d}:=\left\{\left(z_{1}, \ldots, z_{d}\right) \in \mathbb{C}_{p}^{d}: r \leq\left|z_{1}\right|=\ldots=\left|z_{d}\right| \leq r^{\prime}\right\} .
$$

The ring $\mathcal{O}_{K}\left(X_{\left[r, r^{\prime}\right]}^{d}\right)$ of $K$-analytic functions on $X_{\left[r, r^{\prime}\right]}^{d}$ is the ring of all Laurent series

$$
f\left(Z_{1}, \ldots, Z_{d}\right)=\sum_{\alpha \in \mathbb{Z}^{d}} d_{\alpha} \mathbf{Z}^{\alpha}
$$

with $d_{\alpha} \in K$ and such that $\lim _{|\alpha| \rightarrow \infty}\left|d_{\alpha}\right| \rho^{\alpha}=0$ for any $r \leq \rho \leq r^{\prime}$. Here

$$
\mathbf{Z}^{\alpha}:=Z_{1}^{\alpha_{1}} \cdot \ldots \cdot Z_{d}^{\alpha_{d}} \quad \text { and } \quad|\alpha|:=\left|\alpha_{1}\right|+\ldots+\left|\alpha_{d}\right|
$$

(with the abuse of notation that $\left|\alpha_{i}\right|$ exceptionally means the archimedean absolute value). Since $\rho^{\alpha} \leq \max \left(r^{\alpha}, r^{\prime \alpha}\right)$ for any $r \leq \rho \leq r^{\prime}$ and any $\alpha \in \mathbb{Z}^{d}$ the latter condition on $f$ is equivalent to

$$
\lim _{|\alpha| \rightarrow \infty}\left|d_{\alpha}\right| r^{\alpha}=\lim _{|\alpha| \rightarrow \infty}\left|d_{\alpha}\right| r^{\prime \alpha}=0 .
$$

The spectral norm on the affinoid algebra $\mathcal{O}_{K}\left(X_{\left[r, r^{\prime}\right]}^{d}\right)$ is given by

$$
\begin{aligned}
|f|_{X_{\left[r, r^{\prime}\right]}^{d}} & =\sup _{r \leq \rho \leq r^{\prime}} \max _{\alpha \in \mathbb{Z}^{d}}\left|d_{\alpha}\right| \rho^{\alpha} \\
& =\max \left(\max _{\alpha \in \mathbb{Z}^{d}}\left|d_{\alpha}\right| r^{\alpha}, \max _{\alpha \in \mathbb{Z}^{d}}\left|d_{\alpha}\right| r^{\prime \alpha}\right) .
\end{aligned}
$$

Setting $\mathbf{b}^{\alpha}:=b_{1}^{\alpha_{1}} \cdot \ldots \cdot b_{d}^{\alpha_{d}}$ for any $\alpha=\left(\alpha_{1}, \ldots, \alpha_{d}\right) \in \mathbb{Z}^{d}$ we claim that $f\left(b_{1}, \ldots, b_{d}\right):=$ $\sum_{\alpha \in \mathbb{Z}^{d}} d_{\alpha} \mathbf{b}^{\alpha}$ converges in $D_{\left[r, r^{\prime}\right]}(G, K)$. As a consequence of Prop. A.21 and Lemma A.7.iii we have

$$
\left|\mathbf{b}^{\alpha}\right|_{r, r^{\prime}}=\max \left(r^{\alpha}, r^{\prime \alpha}\right)
$$

for any $\alpha \in \mathbb{Z}^{d}$. Hence

$$
\lim _{|\alpha| \rightarrow \infty}\left|d_{\alpha} \mathbf{b}^{\alpha}\right|_{r, r^{\prime}}=\lim _{|\alpha| \rightarrow \infty} \max \left(\left|d_{\alpha}\right| r^{\alpha},\left|d_{\alpha}\right| r^{\prime \alpha}\right)=\max \left(\lim _{|\alpha| \rightarrow \infty}\left|d_{\alpha}\right| r^{\alpha}, \lim _{|\alpha| \rightarrow \infty}\left|d_{\alpha}\right| r^{\prime \alpha}\right)=0 .
$$

Therefore

$$
\begin{aligned}
\mathcal{O}_{K}\left(X_{\left[r, r^{\prime}\right]}^{d}\right) & \longrightarrow D_{\left[r, r^{\prime}\right]}(G, K) \\
f & \longmapsto f\left(b_{1}, \ldots, b_{d}\right)
\end{aligned}
$$

is a well defined $K$-linear map. In order to investigate this map we introduce the filtration

$$
F^{i} D_{\left[r, r^{\prime}\right]}(G, K):=\left\{e \in D_{\left[r, r^{\prime}\right]}(G, K):|e|_{r, r^{\prime}} \leq|p|^{i}\right\} \quad \text { for } i \in \mathbb{R}
$$

on $D_{\left[r, r^{\prime}\right]}(G, K)$. Since $K$ is discretely valued and $r, r^{\prime} \in p^{\mathbb{Q}}$ this filtration is quasi-integral in the sense of [ST5] $\S 1$. The corresponding graded ring $g r^{\cdot} D_{\left[r, r^{\prime}\right]}(G, K)$, by Prop. A.21, is commutative. We let $\sigma(e) \in g r^{\cdot} D_{\left[r, r^{\prime}\right]}(G, K)$ denote the principal symbol of any element $e \in D_{\left[r, r^{\prime}\right]}(G, K)$. 
Proposition A.24. i. $\mathrm{gr}^{\cdot} D_{\left[r, r^{\prime}\right]}(G, K)$ is a free gr $K$-module with basis $\left\{\sigma\left(\mathbf{b}^{\alpha}\right): \alpha \in \mathbb{Z}^{d}\right\}$.

ii. The map

$$
\begin{aligned}
\mathcal{O}_{K}\left(X_{\left[r, r^{\prime}\right]}^{d}\right) & \stackrel{\cong}{\longmapsto} D_{\left[r, r^{\prime}\right]}(G, K) \\
f & \longmapsto f\left(b_{1}, \ldots, b_{d}\right)
\end{aligned}
$$

is a K-linear isometric bijection.

Proof. Since $\left\{s^{-1} \mu: s \in S, \mu \in D_{r}(G, K)\right\}$ is dense in $D_{\left[r, r^{\prime}\right]}(G, K)$ every element in the graded ring $\operatorname{gr}^{\cdot} D_{\left[r, r^{\prime}\right]}(G, K)$ is of the form $\sigma\left(s^{-1} \mu\right)$. Suppose that $\mu=\sum_{\alpha \in \mathbb{N}_{0}^{d}} d_{\alpha} \mathbf{b}^{\alpha}$ and $s=\mathbf{b}^{\beta}$ for some $\beta \in \mathbb{N}_{0}^{d}$. Then $s^{-1} \mu=\sum_{\alpha \in \mathbb{N}_{0}^{d}} d_{\alpha} \mathbf{b}^{-\beta} \mathbf{b}^{\alpha}$ and, using Lemma A.7.iii we compute

$$
\begin{aligned}
\left|s^{-1} \mu\right|_{r, r^{\prime}} & =\max \left(|s|_{r}^{-1}|\mu|_{r},|s|_{r^{\prime}}^{-1}|\mu|_{r^{\prime}}\right) \\
& =\max \left(\max _{\alpha \in \mathbb{N}_{0}^{d}}\left|d_{\alpha}\right| r^{\alpha-\beta}, \max _{\alpha \in \mathbb{N}_{0}^{d}}\left|d_{\alpha}\right| r^{\alpha-\beta}\right) \\
& =\max _{\alpha \in \mathbb{N}_{0}^{d}}\left|d_{\alpha}\right| \max \left(r^{\alpha-\beta}, r^{\alpha-\beta}\right) \\
& =\max _{\alpha \in \mathbb{N}_{0}^{d}}\left|d_{\alpha}\right|\left|\mathbf{b}^{-\beta} \mathbf{b}^{\alpha}\right|_{r, r^{\prime}} .
\end{aligned}
$$

It follows that $g r^{\cdot} D_{\left[r, r^{\prime}\right]}(G, K)$ as a $g r^{\prime} K$-module is generated by the principal symbols $\sigma\left(\mathbf{b}^{-\beta} \mathbf{b}^{\alpha}\right)$ with $\alpha, \beta \in \mathbb{N}_{0}^{d}$. But it also follows that, for a fixed $\beta \in \mathbb{N}_{0}^{d}$, the principal symbols $\sigma\left(\mathbf{b}^{-\beta} \mathbf{b}^{\alpha}\right)$ with $\alpha$ running over $\mathbb{N}_{0}^{d}$ are linearly independent over $g r^{\cdot} K$. By Prop. A.21 we may permute the factors in $\sigma\left(\mathbf{b}^{-\beta} \mathbf{b}^{\alpha}\right)$ arbitrarily. Hence $g r^{\cdot} D_{\left[r, r^{\prime}\right]}(G, K)$ as a $g r^{\cdot} K$-module also is generated by the principal symbols $\sigma\left(\mathbf{b}^{\alpha}\right)$ with $\alpha$ running over $\mathbb{Z}^{d}$. Since any given finite number of the latter can be written in the form $\sigma\left(\mathbf{b}^{-\beta} \mathbf{b}^{\alpha}\right)$ with $\alpha \in \mathbb{N}_{0}^{d}$ and a joint $\beta \in \mathbb{N}_{0}^{d}$ we in fact obtain that $g r^{\cdot} D_{\left[r, r^{\prime}\right]}(G, K)$ is a free $g r^{\cdot} K$-module with basis $\left\{\sigma\left(\mathbf{b}^{\alpha}\right): \alpha \in \mathbb{Z}^{d}\right\}$.

On the other hand, we of course have

$$
\begin{aligned}
f\left(b_{1}, \ldots, b_{d}\right) & \leq \max _{\alpha \in \mathbb{Z}^{d}}\left|d_{\alpha}\right|\left|\mathbf{b}^{\alpha}\right|_{r, r^{\prime}} \\
& =\max _{\alpha \in \mathbb{Z}^{d}}\left|d_{\alpha}\right| \max \left(r^{\alpha}, r^{\prime \alpha}\right)=\max \left(\max _{\alpha \in \mathbb{Z}^{d}}\left|d_{\alpha}\right| r^{\alpha}, \max _{\alpha \in \mathbb{Z}^{d}}\left|d_{\alpha}\right| r^{\prime \alpha}\right) \\
& =|f|_{X_{\left[r, r^{\prime}\right]}^{d}} .
\end{aligned}
$$

This means that if we introduce on $\mathcal{O}_{K}\left(X_{\left[r, r^{\prime}\right]}^{d}\right)$ the filtration defined by the spectral norm then the asserted map respects the filtrations, and by the above reasoning it induces an isomorphism between the associated graded rings. Hence, by completeness of these filtrations, it is an isometric bijection.

If $G$ is commutative the map in Prop. A.24, of course, is an isometric isomorphism of Banach algebras. But in general it is very far from being multiplicative. The graded ring $g r^{\cdot} D_{\left[r, r^{\prime}\right]}(G, K)$ is discussed further in section 4 of the main paper.

One useful consequence of Prop. A.24 is that we have

$$
||_{r, r^{\prime}}=\max \left(||_{r, r},||_{r^{\prime}, r^{\prime}}\right)
$$

on $D_{\left[r, r^{\prime}\right]}(G, K)$. 
The ring $\mathcal{O}_{K}\left(X_{[r, 1)}^{d}\right)$ of $K$-analytic functions on the rigid variety

$$
X_{[r, 1)}^{d}:=\left\{\left(z_{1}, \ldots, z_{d}\right) \in \mathbb{C}_{p}^{d}: r \leq\left|z_{1}\right|=\ldots=\left|z_{d}\right|<1\right\}
$$

is the Fréchet algebra of all Laurent series

$$
f\left(Z_{1}, \ldots, Z_{d}\right)=\sum_{\alpha \in \mathbb{Z}^{d}} d_{\alpha} \mathbf{Z}^{\alpha}
$$

with $d_{\alpha} \in K$ and such that $\lim _{|\alpha| \rightarrow \infty}\left|d_{\alpha}\right| \rho^{\alpha}=0$ for any $r \leq \rho<1$. We also introduce the locally convex $K$-algebra

$$
\mathcal{R}_{K}^{d}:=\underset{r}{\lim } \mathcal{O}_{K}\left(X_{[r, 1)}^{d}\right) .
$$

By limit arguments the map in Prop. A.24 extends to $K$-linear topological isomorphisms

$$
\mathcal{O}_{K}\left(X_{[r, 1)}^{d}\right) \stackrel{\cong}{\longrightarrow} D_{[r, 1)}(G, K)
$$

and

$$
\mathcal{R}_{K}^{d} \stackrel{\cong}{\longrightarrow} R(G, K) .
$$

The coefficients $c_{\beta \gamma, \alpha} \in \mathbb{Q}_{p}$ in the expansions

$$
\mathbf{b}^{\beta} \mathbf{b}^{\gamma}=\sum_{\alpha \in \mathbb{Z}^{d}} c_{\beta \gamma, \alpha} \mathbf{b}^{\alpha} \quad \text { for any } \beta, \gamma \in \mathbb{Z}^{d}
$$

can be viewed in any of the rings under consideration.

Lemma A.25. $\left|c_{\beta \gamma, \alpha}\right| \leq \min \left(1, p^{\alpha-\beta-\gamma}\right)$ for any $\alpha \neq \beta+\gamma$, and $\left|c_{\beta \gamma, \beta+\gamma}-1\right|<1$.

Proof. By Prop. A.21 the coefficients of the expansion

$$
\mathbf{b}^{\beta} \mathbf{b}^{\gamma}-\mathbf{b}^{\beta+\gamma}=\sum_{\alpha \in \mathbb{Z}^{d}} c_{\beta \gamma, \alpha}^{\prime} \mathbf{b}^{\alpha}
$$

satisfy

$$
\left|c_{\beta \gamma, \alpha}^{\prime}\right| r^{\alpha}<\left|\mathbf{b}^{\beta+\gamma}\right|_{r, r}=r^{\beta+\gamma}
$$

for any $1 / p<r<1$ in $p^{\mathbb{Q}}$. Hence $\left|c_{\beta \gamma, \alpha}^{\prime}\right|<r^{\beta+\gamma-\alpha}$. By letting tend $r$ to 1 and $p^{-1}$, respectively, we obtain $\left|c_{\beta \gamma, \alpha}^{\prime}\right| \leq \min \left(1, p^{\alpha-\beta-\gamma}\right)$. For $\alpha=\beta+\gamma$ this means $\left|c_{\beta \gamma, \beta+\gamma}^{\prime}\right|<1$.

\section{A.3 Bounded rings}

In $\mathcal{R}_{K}^{d}$ we have the subrings

$$
\mathcal{R}_{K}^{d, b}:=\left\{f=\sum_{\alpha \in \mathbb{Z}^{d}} d_{\alpha} \mathbf{Z}^{\alpha} \in \mathcal{R}_{K}^{d}:\|f\|:=\sup _{\alpha}\left|d_{\alpha}\right|<\infty\right\}
$$

and

$$
\mathcal{R}_{K}^{d, i n t}:=\left\{f \in \mathcal{R}_{K}^{d}:\|f\| \leq 1\right\} .
$$

It is well known that the norm \|\| on $\mathcal{R}_{K}^{d, b}$ is multiplicative. We let $\mathcal{E}_{K}^{d}$ denote the completion of $\mathcal{R}_{K}^{d, b}$ with respect to \|\| obtaining a $K$-Banach algebra. 
Lemma A.26. $\mathcal{E}_{K}^{d}$ is the ring of all formal series $\sum_{\alpha \in \mathbb{Z}^{d}} d_{\alpha} \mathbf{Z}^{\alpha}$ such that $\sup _{\alpha}\left|d_{\alpha}\right|<\infty$ and $\lim _{\sum \alpha_{i} \leq m,|\alpha| \rightarrow \infty}\left|d_{\alpha}\right|=0$ for any $m \in \mathbb{N}$.

Proof. Let $\widetilde{\mathcal{E}}$ denote the vector space of all these formal series in the assertion. It is easy to see that $\widetilde{\mathcal{E}}$ is complete with respect to \|\| . We also need the subspace $\widetilde{\mathcal{E}}_{0} \subseteq \widetilde{\mathcal{E}}$ of all formal series $\sum_{\alpha \in \mathbb{Z}^{d}} d_{\alpha} \mathbf{Z}^{\alpha}$ such that, for any $m \in \mathbb{N}$, there are only finitely many $\alpha$ with $\sum_{i} \alpha_{i} \leq m$ and $d_{\alpha} \neq 0$.

In a first step we consider an arbitrary element $\sum_{\alpha \in \mathbb{Z}^{d}} d_{\alpha} \mathbf{Z}^{\alpha}$ in $\mathcal{R}_{K}^{d}$. Then $\lim _{|\alpha| \rightarrow \infty}\left|d_{\alpha}\right| r^{\alpha}=$ 0 for some $0<r<1$. It follows that

$$
\lim _{\sum \alpha_{i} \leq 0,|\alpha| \rightarrow \infty}\left|d_{\alpha}\right| \leq \lim _{\sum \alpha_{i} \leq 0,|\alpha| \rightarrow \infty}\left|d_{\alpha}\right| r^{\alpha}=0
$$

and

$$
r^{m} \lim _{\sum \alpha_{i}=m,|\alpha| \rightarrow \infty}\left|d_{\alpha}\right|=\lim _{\sum \alpha_{i}=m,|\alpha| \rightarrow \infty}\left|d_{\alpha}\right| r^{\alpha}=0 \quad \text { for any } m \in \mathbb{N} .
$$

Hence

$$
\lim _{\sum \alpha_{i} \leq m,|\alpha| \rightarrow \infty}\left|d_{\alpha}\right|=0 \quad \text { for any } m \in \mathbb{N} .
$$

In particular, this shows that $\mathcal{R}_{K}^{d, b} \subseteq \widetilde{\mathcal{E}}$.

In a second step we suppose that $\sum_{\alpha \in \mathbb{Z}^{d}} d_{\alpha} \mathbf{Z}^{\alpha}$ lies in $\widetilde{\mathcal{E}}_{0}$. We claim that $\lim _{|\alpha| \rightarrow \infty}\left|d_{\alpha}\right| r^{\alpha}=0$ for any $0<r<1$. Let $\epsilon>0$. We have show that $\left|d_{\alpha}\right| r^{\alpha}<\epsilon$ for all but finitely many $\alpha$. Choose $m \in \mathbb{N}$ in such a way that $\left(\sup _{\alpha}\left|d_{\alpha}\right|\right) r^{m}<\epsilon$. Then certainly $\left|d_{\alpha}\right| r^{\alpha}<\epsilon$ for any $\alpha$ such that $\sum \alpha_{i} \geq m$. But by assumption there are only finitely many nonzero $d_{\alpha}$ with $\sum \alpha_{i} \leq m$. This establishes that $\widetilde{\mathcal{E}}_{0} \subseteq \mathcal{R}_{K}^{d, b}$.

In a third step we argue that $\widetilde{\mathcal{E}}_{0}$ is dense in $\widetilde{\mathcal{E}}$. Let $f=\sum_{\alpha \in \mathbb{Z}^{d}} d_{\alpha} \mathbf{Z}^{\alpha}$ be an arbitrary element in $\widetilde{\mathcal{E}}$. For any $\epsilon>0$ the sets

$$
N_{0}(\epsilon):=\left\{\alpha: \sum \alpha_{i} \leq 0,\left|d_{\alpha}\right| \geq \epsilon\right\} \quad \text { and } \quad N_{m}(\epsilon):=\left\{\alpha: \sum \alpha_{i}=m,\left|d_{\alpha}\right| \geq \epsilon\right\} \text { for } m \in \mathbb{N}
$$

are finite. Hence $f_{\epsilon}:=\sum_{\alpha \in N(\epsilon)} d_{\alpha} \mathbf{Z}^{\alpha}$ with $N(\epsilon):=\bigcup_{m \geq 0} N_{m}(\epsilon)$ lies in $\widetilde{\mathcal{E}}_{0}$ and $\left\|f-f_{\epsilon}\right\|<\epsilon$.

Let $R^{b}(G, K)$ and $R^{\text {int }}(G, K)$ denote the image in $R(G, K)$ of $\mathcal{R}_{K}^{d, b}$ and $\mathcal{R}_{K}^{d, \text { int }}$, respectively, under the above isomorphism. By transport of structure we view both subspaces as normed spaces with respect to \|\| .

Lemma A.27. For any $e \in R(G, K)$ we have

$$
\lim _{r<1, r \rightarrow 1}|e|_{r, r}=\left\{\begin{aligned}
\|e\| & \text { if } e \in R^{b}(G, K), \\
\infty & \text { otherwise. }
\end{aligned}\right.
$$

Proof. Let $0<r_{0}<1$ be such that $e \in D_{\left[r_{0}, 1\right)}(G, K)$. We certainly may assume that $e \neq 0$. Then we may consider the function

$$
\phi(\rho):=\log \left(|e|_{\exp (\rho), \exp (\rho)}\right)
$$

on $\left[\rho_{0}, 0\right)$ where $\rho_{0}:=\log \left(r_{0}\right)$. Let $e=\sum_{\alpha \in \mathbb{Z}^{d}} d_{\alpha} \mathbf{b}^{\alpha}$ and define $N:=\left\{\alpha \in \mathbb{Z}^{d}: d_{\alpha} \neq 0\right\}$. Then

$$
\phi(\rho)=\max _{\alpha \in N}\left(\log \left(\left|d_{\alpha}\right|\right)+\left(\sum_{i} \alpha_{i}\right) \rho\right) .
$$


In particular, as a supremum of affine functions the function $\phi$ is convex on $\left[\rho_{0}, 0\right)$. If $e$ is not in $R^{b}(G, K)$ then $\left\{\log \left(\left|d_{\alpha}\right|\right)\right\}_{\alpha}$ is unbounded which easily implies that $\lim _{\rho<0, \rho \rightarrow 0} \phi(\rho)=\infty$. On the other hand, if $e \in R^{b}(G, K)$ then $\phi$ extends by $\phi(0):=\log (\|e\|)$ to a convex function on $\left[\rho_{0}, 0\right]$, and we have

$$
\log \left(\left|d_{\alpha}\right|\right)+\left(\sum_{i} \alpha_{i}\right) \rho \leq \phi(\rho) \leq \phi(0)+\frac{\phi\left(\rho_{0}\right)-\phi(0)}{\rho_{0}} \rho
$$

for any $\alpha \in N$ and any $\rho \in\left[\rho_{0}, 0\right]$. Let

$$
M:=\left\{\beta \in N:\left|d_{\beta}\right|=\max _{\alpha}\left|d_{\alpha}\right|=\psi(1)\right\} .
$$

We have $\sum_{i} \beta_{i} \geq \frac{\phi\left(\rho_{0}\right)-\phi(0)}{\rho_{0}}$ for any $\beta \in M$. Hence we may choose a $\gamma \in M$ in such a way that $\sum_{i} \gamma_{i}$ is minimal. Then

$$
\log \left(\left|d_{\beta}\right|\right)+\left(\sum_{i} \beta_{i}\right) \rho \leq \log \left(\left|d_{\gamma}\right|\right)+\left(\sum_{i} \gamma_{i}\right) \rho
$$

for any $\beta \in M$ and any $\rho \in\left[\rho_{0}, 0\right]$. On the other hand, if we put

$$
c:=\max \left\{\log \left(\left|d_{\alpha}\right|\right): \alpha \in N \backslash M\right\}
$$

then

$$
\log \left(\left|d_{\alpha}\right|\right)+\left(\sum_{i} \alpha_{i}\right) \rho \leq c+\frac{\phi\left(\rho_{0}\right)-c}{\rho_{0}} \rho
$$

for any $\alpha \in N \backslash M$ and any $\rho \in\left[\rho_{0}, 0\right]$. Altogether we obtain

$$
\phi(\rho) \leq \max \left(c+\frac{\phi\left(\rho_{0}\right)-c}{\rho_{0}} \rho, \log \left(\left|d_{\gamma}\right|\right)+\left(\sum_{i} \gamma_{i}\right) \rho\right)
$$

for any $\rho \in\left[\rho_{0}, 0\right]$. We certainly find a $\rho_{0}<\rho_{1}<0$ such that

$$
c+\frac{\phi\left(\rho_{0}\right)-c}{\rho_{0}} \rho \leq \log \left(\left|d_{\gamma}\right|\right)+\left(\sum_{i} \gamma_{i}\right) \rho
$$

for $\rho \in\left[\rho_{1}, 0\right]$. We conclude that on $\left[\rho_{1}, 0\right]$ the function $\phi$ coincides with the affine function $\log \left(\left|d_{\gamma}\right|\right)+\left(\sum_{i} \gamma_{i}\right) \rho$

Proposition A.28. $R^{b}(G, K)$ and $R^{\text {int }}(G, K)$ are subrings of $R(G, K)$; moreover, the norm \|\| is multiplicative in this ring multiplication.

Proof. For any $f_{1}=\sum_{\beta \in \mathbb{Z}^{d}} c_{\beta} \mathbf{b}^{\beta}$ and $f_{2}=\sum_{\gamma \in \mathbb{Z}^{d}} d_{\gamma} \mathbf{b}^{\gamma}$ in $R(G, K)$ we have

$$
\begin{aligned}
f_{1} f_{2} & =\left(\sum_{\beta} c_{\beta} \mathbf{b}^{\beta}\right)\left(\sum_{\gamma} d_{\gamma} \mathbf{b}^{\gamma}\right)=\sum_{\beta, \gamma} c_{\beta} d_{\gamma} \mathbf{b}^{\beta} \mathbf{b}^{\gamma} \\
& =\sum_{\beta, \gamma} c_{\beta} d_{\gamma} \sum_{\alpha} c_{\beta \gamma, \alpha} \mathbf{b}^{\alpha}=\sum_{\alpha}\left(\sum_{\beta, \gamma} c_{\beta} d_{\gamma} c_{\beta \gamma, \alpha}\right) \mathbf{b}^{\alpha} .
\end{aligned}
$$


If $f_{1}, f_{2} \in R^{b}(G, K)$ we therefore, using Lemma A.25, obtain

$$
\left\|f_{1} f_{2}\right\|=\sup _{\alpha}\left|\sum_{\beta, \gamma} c_{\beta} d_{\gamma} c_{\beta \gamma, \alpha}\right| \leq \sup _{\beta}\left|c_{\beta}\right| \cdot \sup _{\gamma}\left|d_{\gamma}\right|=\left\|f_{1}\right\| \cdot\left\|f_{2}\right\|<\infty
$$

Hence $R^{b}(G, K)$ and $R^{\text {int }}(G, K)$ are subrings and \|\| is submultiplicative. But \|\| , according to Lemma A.27 and Lemma A.22, is a limit of multiplicative norms and therefore is, in fact, multiplicative.

As a consequence of this latter proposition we may complete the algebra $R^{b}(G, K)$ with respect to the norm \| \| obtaining a $K$-Banach algebra $E(G, K)$ with multiplicative norm \|\| . Of course, as Banach spaces, we have our isometric isomorphism

$$
\mathcal{E}_{K}^{d \stackrel{\cong}{\longrightarrow}} E(G, K)
$$

It follows from Lemma A.27 that the rings $R^{b}(G, K), R^{\text {int }}(G, K)$, and $E(G, K)$ together with their norm \||| are independent of the ordering of our chosen basis $h_{1}, \ldots, h_{d}$ of $G$ (because this is the case for the norms ||$_{r, r}$ as we have argued earlier).

The argument in the proof of Lemma A.25 has another interesting consequence. To formulate it we introduce the following convention. Any of the $\operatorname{rings} D_{\left[r_{0}, r\right]}(G, K), D_{\left[r_{0}, 1\right)}(G, K)$, $R(G, K)$, and $E(G, K)$ has its natural multiplication which in general is noncommutative and to which we some times refer as the intrinsic multiplication (always written as $(e, f) \longmapsto e f$ ). But using the bijection from Prop. A.21 and its extensions these rings carry, by transport of structure, also a commutative multiplication which we write as $(e, f) \longmapsto e \circ f$. The notation $e^{-1}$ always will refer to the inverse with respect to the intrinsic multiplication.

Lemma A.29. For any e, $f \in D_{\left[r_{0}, r\right]}(G, K)$ we have

$$
|e f-e \circ f|_{r_{0}, r}<|e f|_{r_{0}, r}=|e \circ f|_{r_{0}, r} .
$$

Proof. Since ||$_{r_{0}, r}=\max \left(||_{\mid r_{0}, r_{0}},||_{r, r}\right)$ it suffices to treat the case $r_{0}=r$. Then the norm is multiplicative so that we have to show that

$$
|e f-e \circ f|_{r, r}<|e|_{r, r}|f|_{r, r} .
$$

Let $e=\sum_{\beta \in \mathbb{Z}^{d}} c_{\beta} \mathbf{b}^{\beta}$ and $f=\sum_{\gamma \in \mathbb{Z}^{d}} d_{\gamma} \mathbf{b}^{\gamma}$. We have

$$
e f=\sum_{\alpha}\left(\sum_{\beta, \gamma} c_{\beta} d_{\gamma} c_{\beta \gamma, \alpha}\right) \mathbf{b}^{\alpha} \quad \text { and } \quad e \circ f=\sum_{\alpha}\left(\sum_{\beta+\gamma=\alpha} c_{\beta} d_{\gamma}\right) \mathbf{b}^{\alpha}
$$

and hence

$$
|e f-e \circ f|_{r, r}=\max _{\alpha}\left|\sum_{\beta+\gamma \neq \alpha} c_{\beta} d_{\gamma} c_{\beta \gamma, \alpha}+\sum_{\beta+\gamma=\alpha} c_{\beta} d_{\gamma}\left(c_{\beta \gamma, \alpha}-1\right)\right| r^{\alpha} .
$$

From the proof of Lemma A.25 we know that

$$
\left|c_{\beta \gamma, \alpha}\right| r^{\alpha}<r^{\beta+\gamma} \quad \text { for } \beta+\gamma \neq \alpha \text { and } \quad\left|c_{\beta \gamma, \beta+\gamma}-1\right|<1 .
$$


We deduce that

$$
\begin{aligned}
|e f-e \circ f|_{r, r} & <\max _{\beta, \gamma}\left|c_{\beta} d_{\gamma}\right| r^{\beta+\gamma} \\
& \leq \max _{\beta}\left|c_{\beta}\right| r^{\beta} \cdot \max _{\gamma}\left|d_{\gamma}\right| r^{\gamma} \\
& =|e|_{r, r} \cdot|f|_{r, r} .
\end{aligned}
$$

Proposition A.30. Let $D$ be any of the rings $D_{\left[r_{0}, r\right]}(G, K), D_{\left[r_{0}, 1\right)}(G, K)$, or $R(G, K)$; we then have:

i. $e \in D$ is a unit with respect to the intrinsic multiplication if and only if it is a unit with respect to the commutative multiplication;

ii. any left or right unit in $D$ is a unit.

Proof. By limit arguments it suffices to consider the case $D=D_{\left[r_{0}, r\right]}(G, K)$. Suppose first that $e$ is a commutative unit, i.e., $e \circ f=1$ for some $f \in D$. By Lemma A.29 we then have $|e f-1|_{r_{0}, r}<1$ which implies, since we are in a Banach algebra, that $e f$ is an intrinsic unit. Starting from $f \circ e=1$ we similarly obtain that $f e$ is an intrinsic unit. Hence $e$ and $f$ are intrinsic units. Now let, vice versa, $e$ be a left intrinsic unit (the case of a right one being analogous), say, $e f=1$ for some $f \in D$. By a totally analogous reasoning as above we obtain that $e \circ f=f \circ e$ and hence $e$ and $f$ are commutative units. But then we actually may apply the first part of the proof to conclude that $e$ is an intrinsic unit.

Proposition A.31. i. $R^{b}(G, K) \cap R(G, K)^{\times}=R^{b}(G, K)^{\times}$;

ii. an element in $R^{b}(G, K)$ is a unit with respect to the intrinsic multiplication if and only if it is a unit with respect to the commutative multiplication.

Proof. i. Let $e \in R(G, K)^{\times}$such that $\|e\|<\infty$. Suppose that $e \in D_{\left[r_{0}, 1\right)}(G, K)^{\times}$. By Lemma A.27 and its proof we know that $\lim _{r<1, r \rightarrow 1}|e|_{r, r}=\|e\|$ and that the function $\phi(\rho):=$ $\log \left(|e|_{\exp (\rho), \exp (\rho)}\right)$ is an affine function on $\left[\rho_{1}, 0\right]$ for $\rho_{1}$ sufficiently close to 1 . Since the norms | $\mid \exp (\rho), \exp (\rho)$, by Lemma A.22, are multiplicative on $D_{\left[r_{0}, 1\right)}(G, K)$ it follows that

$$
\lim _{\rho<0, \rho \rightarrow 0} \log \left(\left|e^{-1}\right|_{\exp (\rho), \exp (\rho)}\right)=-\lim _{\rho<0, \rho \rightarrow 0} \phi(\rho)=-\log (\|e\|)<\infty .
$$

Hence, again by Lemma A.27, we have $e^{-1} \in R^{b}(G, K)$. ii. This follows from Prop. A.30, i by applying the present assertion i. to $G$ as well as the commutative group $\mathbb{Z}_{p}^{d}$.

Lemma A.32. We have $h_{i}^{x}-1 \in R^{i n t}(G, K)^{\times}$for any $x \in \mathbb{Z}_{p} \backslash\{0\}$ and any $1 \leq i \leq d$.

Proof. Write $x=p^{m} y$ with $y \in \mathbb{Z}_{p}^{\times}$. We know from (49) that $(1+Z)^{y}-1 \in Z \cdot \mathbb{Z}_{p}[[Z]]^{\times}$and hence $(1+Z)^{x}-1 \in\left[(1+Z)^{p^{m}}-1\right] \cdot \mathbb{Z}_{p}[[Z]]^{\times}$. Moreover the leading coefficient of $(1+Z)^{p^{m}}-1$ is equal to 1 . Hence

$$
h_{i}^{x}-1 \in\left(h_{i}^{p^{m}}-1\right) \cdot R^{i n t}(G, K)^{\times} \quad \text { and } \quad\left\|h_{i}^{x}-1\right\|=1 .
$$

By Prop. A.31,i and the multiplicativity of \|\| it now suffices to show that $h_{i}^{p^{m}}-1$ is invertible in $R(G, K)$. But the polynomial $(1+Z)^{p^{m}}-1$ has no zeros in an appropriate annulus $r_{m} \leq|z|<1$. This implies that $h_{i}^{p^{m}}-1 \in D_{\left[r_{0}, 1\right)}(G, K)^{\times}$. 
The formula (50) implies that the rings $R^{i n t}(G, K) \subseteq R^{b}(G, K) \subseteq E(G, K)$ together with the norm \|\| do not change if we replace the generating set $\left\{h_{1}, \ldots, h_{d}\right\}$ by $\left\{h_{1}^{x_{1}}, \ldots, h_{d}^{x_{d}}\right\}$ for some $x_{1}, \ldots, x_{d} \in \mathbb{Z}_{p}^{\times}$.

\section{References}

[Ba] Bass H., K-theory and stable algebra, Publ. Math. I.H.E.S. 22 (1964), 5-60.

[BHS] Bass H., Heller A., Swan R., The Whitehead group of a polynomial extension, Publ. Math. I.H.E.S. 22 (1964), 61-80.

[B-AC] Bourbaki N., Algèbre commutative, Hermann, Paris.

[B-GT] Bourbaki N.: General Topology. Berlin-Heidelberg-New York: Springer 1989

[DDMS] Dixon J.D., du Sautoy M.P.F., Mann A., Segal D.: Analytic Pro-p-Groups. Cambridge Univ. Press 1999

[GaRe] Gabriel P., Rentschler R., Sur la dimension des anneaux et ensembles ordonnés, $C$. R. Acad. Sci. Paris Sér. A-B 265 (1967) A712-A715.

[GiRo] Gilles C., Robba P., Equations differentielles p-adiques, Hermann, Paris.

[G] Gruson L., Fibrés vectoriels sur un polydisque ultramétrique, Ann. Sci. École Norm. Sup. (4) 1 (1968), 45-89.

[LvO] Li H., van Oystaeyen F., Zariskian filtrations, Kluwer Academic Publishers, Kmonographs in Mathematics, vol.2 (1996).

[MR] McConnell J. C., Robson J. C., Noncommutative Noetherian rings, AMS Graduate Studies in Mathematics, vol. 30.

[S1] Schmidt T., Auslander regularity of $p$-adic distribution algebras, Represent. Theory 12 (2008), 37-57.

[S2] Schmidt T., Vector bundles over analytic character varieties, preprint, http://arxiv.org/abs/0903.4917

[ST1] Schneider P., Teitelbaum J., $U(\mathfrak{g})$-finite locally analytic representations, Represent. Theory 5 (2001), 111-128.

[ST2] Schneider P., Teitelbaum J., p-adic Fourier theory, Doc. Math. 6 (2001), 447-481.

[ST3] Schneider P., Teitelbaum J., Locally analytic distributions and $p$-adic representation theory, with applications to $\mathrm{GL}_{2}$, J. Amer. Math. Soc. 15 (2002), 443-468.

[ST4] Schneider P., Teitelbaum J., Banach space representations and Iwasawa theory, Israel J. Math. 127 (2002), 359-380.

[ST5] Schneider P., Teitelbaum J., Algebras of $p$-adic distributions and admissible representations, Invent. Math. 153 (2003), 145-196. 
[ST6] Schneider P., Teitelbaum J., Continuous and locally analytic representation theory, Notes of lectures held at Hangzhou, August 2004, http://wwwmath.uni-muenster.de/u/schneider/publ/lectnotes/index.html

[ST7] Schneider P., Teitelbaum J., Duality for admissible locally analytic representations, Representation Theory $\mathbf{9}$ (2005), 297-326.

[Spr] Springer T.A.: Microlocalisation algebrique, in Sém. d'Algèbre Paul Dubreil et M.-P. Malliavin 1984, Lecture Notes Math. 1146, 299-316. Berlin-Heidelberg-New York: Springer 1985

[St] Stafford J. T., On the stable range of right Noetherian rings, Bull. London Math. Soc. 13 (1981), no. 1, 39-41.

[vdE] van den Essen A.: Algebraic Micro-Localization, Communic. Alg. 14 (1986), 9711000 . 